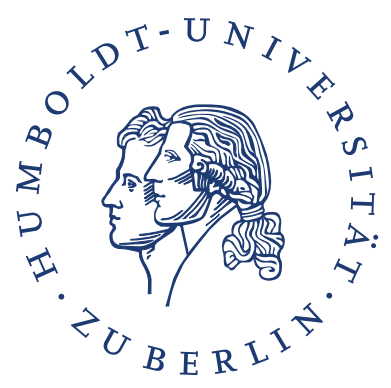

\title{
Coupling Analysis of Transient Cardiovascular Dynamics
}

\author{
DISSERTATION \\ zur Erlangung des akademischen Grades \\ doctor rerum naturalium (Dr. rer. nat.) \\ im Fach Physik \\ eingereicht an der Mathematisch-Naturwissenschaftlichen Fakultät der \\ Humboldt-Universität zu Berlin \\ von \\ Diplom-Physiker \\ Andreas Müller
}

Präsident der Humboldt-Universität zu Berlin:

Prof. Dr. Jan-Hendrik Olbertz

Dekan der Mathematisch-Naturwissenschaftlichen Fakultät:

Prof. Dr. Elmar Kulke

Gutachter:

1. Prof. Dr. Dr. h.c. mult. Jürgen Kurths

2. Prof. Dr. Thomas Penzel

3. Assoc. Prof. Dr. Mathias Baumert

Tag der mündlichen Prüfung:

09.02 .2016 



\section{Zusammenfassung}

Die Untersuchung kausaler Zusammenhänge in komplexen dynamischen Systemen spielt in der Wissenschaft eine immer wichtigere Rolle. Ziel dieses aktuellen, interdisziplinären Forschungsbereiches ist ein grundlegendes, tiefes Verständnis der vorherrschenden Prozesse und deren Wechselwirkungen in solchen Systemen. Anwendungen stammen dabei aus Bereichen wie der Klimaforschung, der globalen ökonomischen Systeme, des menschlichen Herzkreislaufsystems und der neurophysiologischen Prozesse im Gehirn. Die Untersuchung von Zeitreihen aus diesen Gebieten mithilfe moderner Kopplungsanalysemethoden liefert dabei Möglichkeiten zur Modellierung der betreffenden Systeme und somit bessere Vorhersagemethoden und fortgeschrittene Interpretationsmöglichkeiten der Ergebnisse. So wurden in letzter Zeit unter anderem bekannte und neu gefundene Zusammenhänge zwischen Prozessen des Klimasystems, z.B. zwischen der El Niño Southern Oscillation (ENSO) und dem indischen Monsun, sowie kurzfristige kardiovaskuläre Regulationen mithilfe solcher Methoden erfolgreich genauer untersucht und interpretiert. Bei der Anwendung von Kopplungsanalysemethoden für Zeitreihen ist es jedoch wichtig, zwischen dem alltäglichen Kausalitätsbegriff und dem Begriff der Kopplung zu unterscheiden. In den letzten Jahren wurde eine Fülle von Werkzeugen entwickelt, die oft auf dem Prinzip der Granger-Kausalität oder anderen abgeschwächten Versionen einer Kausalitätsdefinition beruhen.

Unter den oben genannten Systemen ist besonders das menschliche Herz-Kreislauf-System, dessen komplexes Verhalten durch viele sich überlagernde Regulierungsmechanismen bestimmt wird, ein aktuelles Forschungsgebiet. Ein wichtiges Teilgebiet umfasst dabei den Bereich der Interaktionen zwischen Schlag-zu-Schlag-Intervallen und dem Blutdruck, die einerseits zumindest teilweise bekannt sind, andererseits aber bis heute noch kontrovers diskutiert werden.

In der vorliegenden Arbeit werden zunächst einige existierende Kopplungsmaße mit ihren jeweiligen Anwendungsgebieten vorgestellt. Eine Gemeinsamkeit dieser Maße liegt in der Voraussetzung stationärer Zeitreihen, um die Anwendbarkeit zu gewährleisten. Daher wird im Verlauf der Dissertation eine Möglichkeit zur Erweiterung solcher Maße vorgestellt, die eine Kopplungsanalyse mit einer sehr hohen Zeitauflösung und somit auch die Untersuchung nichtstationärer, transienter Ereignisse ermöglicht.

Die Erweiterung basiert auf der Verwendung von Ensembles von Messreihen und der Schätzung der jeweiligen Maße über das Ensemble anstatt über die Zeit. Dies ermöglicht eine Zeitauflösung bei der Analyse in der Größenordnung der Abtastrate des ursprünglichen Signals, die nur von der Art der verwendeten Kopplungsmaße abhängt.

Die Ensemble-Erweiterung wird auf verschiedene Kopplungsmaße angewandt, die bereits er- 
folgreich unter schwierigeren Bedingungen wie starkem Rauschen oder kurzen Messreihen verwendet wurden. Zum Vergleich werden zwei einfachere Kopplungsmaße herangezogen. Zunächst werden die Methoden ausführlich an verschiedenen theoretischen Modellen und unter verschiedenen Bedingungen getestet. Anschließend erfolgt eine zeitaufgelöste Kopplungsanalyse kardiovaskulärer Zeitreihen, die während transienter Ereignisse aufgenommen wurden. Die Ergebnisse dieser Analyse bestätigen zum einen aktuelle Studienresultate, liefern aber auch neue Erkenntnisse, die es in Zukunft ermöglichen können, Modelle des Herz-Kreislauf-Systems zu erweitern und zu verbessern. Auf diesen Modellen basierende, bessere Vorhersagemethoden können anschließend neue Diagnoseverfahren und Behandlungsmethoden kardiovaskulärer Erkrankungen erlauben und somit einen wichtigen Beitrag zur Gesundheitserhaltung des Menschen liefern. 


\section{Summary}

The analysis of causal relationships in complex dynamic systems plays a more and more important role in various scientific fields. The aim of this current, interdisciplinary field of research is a fundamental, deep understanding of predominant processes and their interactions in such systems. Frequent applications stem from fields like climate research, global economics systems, the human cardiovascular system, and neurophysiological processes in the brain. The study of time series originating from these areas using modern coupling analysis tools allows the modelling of the respective systems and thus better prediction methods and advanced interpretation possibilities for the results. Recently, coupling measures have been used to analyse and interpret, among others, known and newly found connections between climate system processes like the El Niño Southern Oscillation (ENSO) and the Indian monsoon, and short-term cardiovascular regulations. When applying such measures it is important to distinguish between the common concept of causality and the term of coupling. During the last years, a plethora of coupling measures has been developed, often based on the Granger causality principle or on other attenuated forms of common causality definitions.

Among the systems mentioned above, especially the cardiovascular system, whose complex behaviour is determined through many overlapping regulatory processes, is an ongoing research topic. An important section of this field comprises the interactions between the beat-to-beat intervals and the blood pressure, which on the one hand are at least known partially and on the other hand are controversially discussed.

In this work, initially some existing coupling measures and their fields of application are introduced. One trait these measures have in common is the requirement of stationary time series to ensure their applicability. Therefore, in the course of this thesis a possibility to extend these measures is presented, which allows a coupling analysis with a high temporal resolution and thus also the analysis of transient, nonstationary events.

The extension is based on the use of ensembles of time series and the calculation of the respective measures across these ensembles instead of across time. This allows for a temporal resolution of the same order of magnitude as the sampling rate in the original signal. The resolution only depends on the kind of coupling analysis method employed.

The ensemble extension is applied to different coupling measures already successfully employed under difficult circumstances like high noise levels or short time series. For comparison, two simpler coupling measures are used. To begin with, the regarded tools are tested on various theoretical models and under different conditions. This is followed by a coupling analysis of cardiovascular time series recorded during transient events. The results on the one hand 
confirm topical study outcomes and on the other hand deliver new insights, which will allow to extend and improve cardiovascular system models in the future. Prediction methods based on these models will then be able to provide new diagnostic techniques and treatment procedures for cardiovascular diseases, thus contributing to health preservation in humans. 


\section{Acknowledgements}

I wish to express my sincere thanks to my advisors Prof. Jürgen Kurths and PD Dr. Niels Wessel for their support, opportunities of discussion, and for giving me the freedom to follow my own ideas. I am indebted to the DFG (Deutsche Forschungsgemeinschaft) for providing the funding for my work via the DFG grant WE2834/5-1. Special thanks go to the other members of the Cardiovascular Physics group of the Humboldt-Universität zu Berlin, Maik Riedl and Jan Krämer, as well as the numerous guests, for the fruitful discussions especially during the legendary coffee breaks.

Additionally, I thank the whole group of Prof. Kurths at the Potsdam Institute for Climate Impact Research (PIK) and especially Dr. Norbert Marwan for the interesting discussions. I also want to thank Prof. Thomas Penzel from the Sleep Medicine Center of the Charité Berlin and Prof. Hendrik Bonnemeier from the Universitätsklinikum Schleswig-Holstein for providing the data for my work.

Great appreciation goes to my family for supporting me during my studies and this work. And finally, thank you, Janina, for always believing in me. 



\section{Contents}

$\begin{array}{ll}\text { Zusammenfassung } & 3\end{array}$

Summary 5

$\begin{array}{ll}\text { Acknowledgements } & 7\end{array}$

1 Introduction $\quad 11$

2 Methods $\quad 18$

2.1 Ensemble approach . . . . . . . . . . . . . . . . . . . . . 18

2.2 Classical measures . . . . . . . . . . . . . . . . . . . . . . . . 21

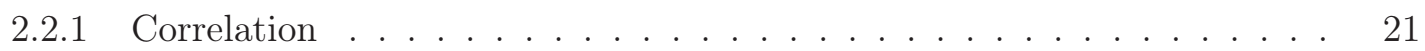

2.2 .2 Mutual information . . . . . . . . . . . . . . . . . 21

2.2.3 Ensemble cross-correlation (EXCORR) and ensemble mutual information

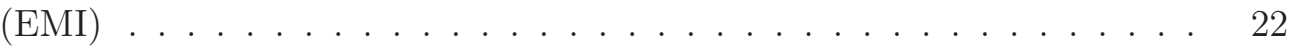

2.3 Granger causality . . . . . . . . . . . . . . . . . . . . . . . 23

2.3.1 Classical Granger causality . . . . . . . . . . . . . . . . 23

2.3.2 Conditional Granger causality . . . . . . . . . . . . . . . 25

2.3.3 Partial Granger causality . . . . . . . . . . . . . . . . . 26

2.3.4 Ensemble Granger causality (EGC) . . . . . . . . . . . . . . . . . 27

2.4 Symbolic coupling traces . . . . . . . . . . . . . . . . . . . . . . . . . . . . 29

2.4.1 Classic symbolic coupling traces . . . . . . . . . . . . . . . . . . . . 29

2.4.2 Permutation symbolic coupling traces . . . . . . . . . . . . . . . . 31

2.4.3 Ensemble symbolic coupling traces (ESCT and PESCT) . . . . . . . . . 32

3 Applications to models $\quad 34$

3.1 Analysis . . . . . . . . . . . . . . . . . . . . . . 34

3.1 .1 AR-models . . . . . . . . . . . . . . . . . . 34

3.1 .2 Nonlinear models . . . . . . . . . . . . . . . . . . . . . . . . . . . 41

3.1.3 Influence of ensemble size, coupling strength, and noise . . . . . . . . . . 49

3.2 Discussion . . . . . . . . . . . . . . . . . . . . . 52

4 Application to data measured during an orthostatic test 55

4.1 Analysis . . . . . . . . . . . . . . . . . . . . . 5 5

4.2 Discussion . . . . . . . . . . . . . . . . . . . . 64 
5 Application to data measured during sleep arousals $\quad 67$ 5.1 Analysis . . . . . . . . . . . . . . . . . . . . . 6 67

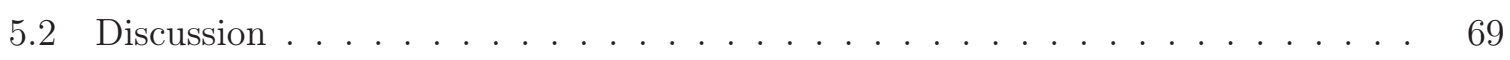

6 Conclusion \& Outlook $\quad 72$

$\begin{array}{ll}\text { List of Abbreviations } & 75\end{array}$

$\begin{array}{ll}\text { List of Frequently used mathematical symbols } & 76\end{array}$

$\begin{array}{ll}\text { List of Figures } & 77\end{array}$

$\begin{array}{ll}\text { List of Tables } & 78\end{array}$

$\begin{array}{ll}\text { List of Publications } & 79\end{array}$

$\begin{array}{lr}\text { Bibliography } & 80\end{array}$ 


\section{Introduction}

A lot of people throughout the world suffer from cardiovascular diseases, which are the number one cause of death worldwide [WHO10]. Their treatment causes enormous costs for the public health care system $\left[\mathrm{HTK}^{+} 11\right]$ and is not always successful. These are among the main reasons why the study of the human cardiovascular system plays such a big role in the field of medical science, which can look back on a history of over one hundred years. The comparatively new branch of Cardiovascular Physics [WKDB07], which combines methods from linear and nonlinear data analysis and modelling with medical background knowledge, has brought forth a lot of new interesting insights and tools to help in understanding the interactions of the cardiovascular system and thus predicting diseases, assessing risks and providing new clinical parameters $\left[\mathrm{CFB}^{+} 93, \mathrm{DB} 92, \mathrm{HKvdL}^{+} 94, \mathrm{MWH}^{+}\right.$02, $\left.\mathrm{SAL}^{+} 89\right]$. The development of noninvasive measurements like the electrocardiogram (ECG) [Ein03], the echocardiogram [Bak70], the magneto-resonance tomography [NVF02] and blood pressure recording using a sphygmomanometer [Mil51], in the last century, allows for a far easier way to get data without greatly impeding the subjects. Today, the most often applied technique is measuring the ECG, because of the simple measurement setup and its cost efficiency. It is characterised by a unique waveform depicting the P-wave (atrial excitation) followed by the QRS-complex (fast depolarisation) with the distinctive R-peak, and the T-wave (ventricular repolarisation)[Lev10]. From the ECG, variables like the beat-to-beat intervals (BBI) or their inverse, the heart rate, the heart's electrical axis in the frontal plane and the electrical activity of the atria and the ventricles can be derived. Just relying on the ECG, it is already possible to diagnose abnormal rhythms caused by damage of the tissue, which carries the electrical signals, or non-cardiac conditions [VSK04] like heart blocks [Lew20], myocardial infarction [GFPK84], hypertrophy [Kli97], electrolyte abnormalities [Sur95], pulmonary embolism [ $\left.\mathrm{UBP}^{+} 01\right]$, and hypothermia [GBEC95]. Another measurement is the beat-to-beat blood pressure recording, whose most important development stations encompass among others the hydraulic function of the cardiovascular system [Har28], the realisation that respiration causes blood pressure fluctuations [Hal33, Lud45], and the discovery of rhythmic blood pressure fluctuations, the so-called Mayer waves [May76]. Blood pressure is usually described by the two variables of systolic and diastolic blood pressure, i.e. the maximum (contraction of the left ventricle) and the minimum (permanent pressure without heart activity) value of the blood pressure curve during a beat-to-beat interval, respectively. The introduction of non-invasive measurement methods [Mil51, Pen73] has rendered the blood pressure to be one of the most important signals in the evaluation of physiological questions. Today a continuous measurement with only low restrictions for the subjects is possible using portable devices. Pre- 
vious studies have shown that the blood pressure signal shows large variations during the day and even during longer periods like days, months, or seasons $\left[\mathrm{MFG}^{+} 83, \mathrm{MMB}^{+} 06, \mathrm{SCB}^{+} 98\right]$ providing important clinical parameters $\left[\mathrm{MOP}^{+} 01, \mathrm{MOR}^{+} 95, \mathrm{MPB}^{+} 07\right]$. Relationships between cardiovascular conditions $\left[\mathrm{KOA}^{+} 05, \mathrm{STF}^{+} 99\right]$ and organ damage related to hypertension [FST97, $\mathrm{MPH}^{+}$01] have been found via cross-sectional and longitudinal studies. Other parameters like the stroke volume (SV), the volume of blood pumped from one ventricle of the heart per beat, and the cardiac output (CO), the blood volume pumped by the heart per minute, are usually either measured using invasive methods or by an echocardiogram [Lev10]. However, based on the blood pressure measurement and knowledge about the cardiovascular system, it is also possible to estimate parameters like $\mathrm{SV}$ or $\mathrm{CO}$ on a beat-to-beat basis. The SV can for example be computed by integrating the aortic flow waveform per beat, which in turn is estimated from the arterial pressure signal using a nonlinear model [ $\mathrm{JIO}^{+} 99$, WJSS93]. The $\mathrm{CO}$ can then be simply calculated as the product of heart rate and the SV values.

The aforementioned development of non-invasive tools to measure physiological signals, e.g. the ECG and the blood pressure, has led to an enormous amount of data recorded under various conditions. The challenge now lies in analysing the data, thus trying to understand the underlying mechanisms and their interactions amongst each other, and in the end extracting meaningful parameters usable for diagnostics and risk stratification. For example, heart rate (HRV) and blood pressure variability (BPV) parameters have helped understanding the nervous control mechanisms of the cardiovascular system [AGU ${ }^{+} 81$, LV76, Say73, Tas96]. However, the many open questions lead to an undamped interest in analysing the data and developing new sophisticated methods. Due to the complex structure with its many control loops and the strong dependence on internal as well as external conditions, the cardiovascular system exhibits a complicated spatio-temporal behaviour. Thus, a lot of fruitful ideas have been contributed by the field of chaos theory and nonlinear dynamics during the last decades $\left[\mathrm{KSA}^{+}\right.$04, Lom00, Mal98, $\mathrm{MWM}^{+}$02, $\mathrm{PFM}^{+} 07, \mathrm{SDHK05}, \mathrm{SRAK} 9, \mathrm{VKK}^{+}$95, $\mathrm{VKK}^{+}$96, WMBK07]. In order to gain a deeper insight into the actual mechanisms purely descriptive linear or nonlinear parameters are not sufficient, mathematical models are needed. Using these it is possible to describe the individual components and their interactions under various conditions, for example during diseases, and finally to draw conclusions about the reality [CT02]. Usually, there are two approaches. The first one uses differential [CB96, Gro59, $\mathrm{KST}^{+} 05, \mathrm{Kuu} 04, \mathrm{OPK}^{+} 00, \mathrm{Ott97}, \mathrm{ZGB}^{+}$07] or difference equations [DKS87, RK95] based on principles of physics, mathematics and, in this case, incorporating knowledge of physiology about couplings between e.g. heart rate, blood pressure, and respiration. The second one employs tools from time series analysis and system identification to model the measured data via autoregressive (AR) models and thus infer mechanisms independent of a priori knowledge $\left[\mathrm{BCB}^{+} 94, \mathrm{CMT}^{+} 97, \mathrm{MW} 97, \mathrm{PBR}^{+} 00, \mathrm{PLG}^{+} 86\right]$. A 
problem with this approach lies in the potentially large number of possible parameters, which might interfere with a physiological interpretation. Also, as most natural processes, the cardiovascular system exhibits highly nonlinear behaviour, impairing the use of linear methods and models without further effort. For this reason, several extensions for nonlinear AR-models to describe HRV and BPV have been proposed in the last years, e.g. bilinear [AJI ${ }^{+} 02$ ], functional coefficient [BBSK02], nonlinear additive AR-models without (NAAR) $\left[\mathrm{WMB}^{+} 06\right]$ and with external input (NAARX) [Rie09, $\mathrm{RSM}^{+}$08], and AR-models with conditional heteroscedasticity [KHI03].

For the models to help us in understanding the underlying mechanisms, we need to identify the interactions between the single variables using no or only little a priori knowledge. Therefore, a plethora of coupling measures to allow for identifying a complex system's coupling structure, including coupling strength, direction, and occurring time lags, has been developed over the years.

\section{Coupling measures}

The analysis of effects from coupling in and between systems is important in data-driven investigations as practised in many scientific fields. It allows deeper insights into the mechanisms of interaction emerging among individual smaller subsystems when forming complex systems as in the human circulatory system or the climate system. In the last century and especially during the last 20 years the development and application of coupling measures became more and more important. The correct application of those, requires at least a basic understanding of the concept of causality. Since there is no binding definition of the term causality, two examples are given here.

An event $A$ is said to be causal for an event $B$ if,

- when $A$ happens, $B$ also takes place (necessary criterion),

- $A$ happens chronologically before $B$,

- and, if $A$ does not happen, $B$ cannot occur either (sufficient criterion).

Based on probability theory also the next definition is possible. $A$ causes $B$, if

- the probability for $A$ to occur is not zero,

- $A$ happens chronologically before $B$,

- and the probability for $B$ to happen, when $A$ has occurred before, is larger than the probability of $B$ taking place on its own. 
Due to the relativity theory, the second point in both definitions implies also a spatial restriction, which can be neglected for a lot of applications of coupling analyses, however. The utilisation of these definitions for time series analysis is not readily feasible. Often, some measure of $a$ priori knowledge is still needed. One attempt of a causality definition for time series analysis was given by Granger [Gra69]. A process $X$ Granger-causes a process $Y$, if

- $X$ happens chronologically before $Y$

- and the error when predicting the future of $Y$ is reduced when taking information from $X$ into account.

A lot of coupling measures are based on this definition. However, there are also other measures which employ another definition. A process $X$ influences a process $Y$, if

- $X$ happens chronologically before $Y$

- and the processes show similar behaviour.

Of course, these definitions are strongly attenuated versions of the causality definitions above. Therefore, one has to keep in mind, that usually a found coupling in time series can imply a causal connection, but cannot be taken as compelling proof. At least not, if not all variables of a given complex system are known.

While often classic methods like correlation and coherence are used to define connections between subsystems (compare e.g. [ $\left.\mathrm{NFP}^{+} 05, \mathrm{RGAC} 14\right]$ for cortex networks and the cardiovascular system), today, there are coupling measures originating in different fields comprising Granger causality, methods based on information theory, phase space measures, symbolic dynamics, and synchronisation and coordination, which are able to provide more information about coupling strength and direction. There are several works comparing the different measures and testing their applicability in different situations stemming from neurophysiological and cardiovascular systems [Leh11, LIKO07, PF13, $\left.\mathrm{SAE}^{+} 13\right]$. Several models of the cardiovascular system have been proposed based on the results of combining practical and theoretical a priori knowledge with insights obtained via coupling analyses [DKS87, $\mathrm{PBR}^{+} 00, \mathrm{PFR}^{+}$02, SLM01, SLSH01, $\left.\mathrm{SNG}^{+} 04\right]$. In the next subsections some of the existing coupling measures will be briefly introduced and examples of systems they have been applied to are given.

\section{Granger causality}

The classical Granger causality was introduced in [Gra69]. It is based on estimating ARmodels for the data given and checking whether the errors produced by the modelling process are significantly reduced when incorporating information from a second variable. Over the 
years, several extensions for multivariate data [Gra69, Gew84, GSK ${ }^{+}$08], later in this work discussed in more detail, and nonlinear applications have been developed. This includes the use of NAARX-models [FNC08, Rie09, $\mathrm{RSM}^{+} 08, \mathrm{RSS}^{+}$10], different embedding techniques [CRFD04, IOLK08b], the use of radial basis functions [AMS04], and the application of kernel based methods [MLCS11, MPS08b, MPS08a]. A comparison of different nonlinear extensions can be found in [HSPVB07, IOLK08a]. The applications range from financial data over cardiovascular, neurophysiological, and gene regulatory network data to climate time series. To assess also long-term couplings for example in climate data, in [SM09] an appropriate approach has been proposed. The spectral version of Granger causality is also known as partial directed coherence and has mainly been applied measurements of the electrical activity of the brain (electroencephalogram, EEG) [BS01, $\mathrm{SWE}^{+}$06, WSH ${ }^{+}$06, WST07].

\section{Information theoretic measures}

The methods stemming from the field of information theory are usually based on a form of mutual information [Sha48]. The first subgroup is the transfer entropy [Sch00b] with several extensions [LPK07, Ver05]. It has been mostly applied to cardiovascular data. The second measure, the conditional mutual information [Pal96], bears some similarities with the transfer entropy and is in some cases equivalent. It has been widely applied to neurophysiological and cardiovascular data [FP07, FNP11, FNP12, $\mathrm{MSM}^{+}$07, Pal07, PKHv01, $\mathrm{PKP}^{+}$01, PS03, PSV04, PV07, QCKH11, SB14, SCB14, STB14, Vej08, VP08]. This approach can also be used on phase time series. An overview about several information theoretic methods can be found in [HSPVB07]. Recently, a new approach, the so-called momentary information transfer, has been introduced. It specialises on avoiding spurious couplings by conditioning on certain subgroups of the data points and on how to identify these. It has been successfully applied to climate and cardiovascular data [PR11, RHMK12, RHPK12], [9].

\section{Phase space measures}

The phase space methods are usually based on mutual prediction using a nearest neighbours approach and comparing prediction errors when incorporating other variables [QAG00, $\left.\mathrm{SSC}^{+} 96\right]$. Thus, they are also based on identifying causalities in the sense of Granger. There are today several refinements of the original measures using e.g. rank statistics, and they have been successfully applied to different nonlinear model systems and neurophysiological as well as cardiovascular data [AGLE99, AK11, CA09, FPN08, LMAV99, NFAP09, QKKG02, SA05, Sch00a, TB03]. A second class in this field consists of recurrence based measures with applications to climate series and the cardiovascular system $\left[\mathrm{FDD}^{+} 12, \mathrm{MZW}^{+} 13, \mathrm{RGM}^{+} 13, \mathrm{RTKG} 7, \mathrm{ZRT}^{+} 11\right]$. Among these measures there is also an approach to identify hidden variables to avoid spurious 
connections [HA10].

\section{Symbolic dynamics}

Among other features, their robustness against noise predestines symbolic approaches for a coupling analysis. They are based on the symbolification of the data using different approaches. The coupling analysis part is usually done by applying another known coupling measure algorithm on the obtained symbol sequences. By choosing the symbol alphabet, word length, and time lags between consecutive 'letter' of a word, one can easily adapt the measures to the needs at hand (e.g. short-term or long-term coupling). Some of the most successful measures are the symbolic transfer entropy [SL08, SL09a], joint symbolic dynamics [STB ${ }^{+}$13], and the symbolic coupling traces $\left[\mathrm{SRM}^{+} 10, \mathrm{WSR}^{+} 09\right]$, which have all been applied to neurophysiological and cardiovascular data and have delivered promising results. From these measures, the symbolic coupling traces are discussed in the next chapter in more detail.

\section{Synchronisation \& coordination}

Synchronisation [PRK01] is an effect which usually renders the detection of coupling directions impossible, since in a completely synchronised state two systems cannot be distinguished anymore. However, using approaches based on synchronisation detection it is possible to perform a coupling analysis for systems not completely synchronised. One of these approaches is the so-called evolution map approach [RP01] which has been extensively used on theoretic models and EEG as well as cardiorespiratory data [BPRP03, $\mathrm{CRF}^{+} 03, \mathrm{MCPR} 03, \mathrm{MSM}^{+} 07, \mathrm{RCB}^{+} 02$, SA05, SB03]. A second tool, the synchrogram [SRKA98], allows for a graphical interpretation of synchronised states in bivariate systems. It has been mainly used on cardiorespiratory data [MPR00, RKP ${ }^{+}$98, RPK ${ }^{+}$01, SRAK99, SRKA98, SWD ${ }^{+}$06]. Since this measure is used to detect phase synchronisation, it is not a coupling measure per se, but still has delivered interesting insights. However, another method based on a similar approach, namely the coordigram [6], can be used to infer coupling directions. As opposed to the synchronisation, which describes a phase-based relationship between systems, the coordination describes a time-based connection (e.g. between the time points of the onsets of respiratory cycles and the heart beats) and has been shown to play an important role for example in cardiorespiratory mechanisms [Ras86, Ras87, RH82].

\section{Subject of this thesis}

An important issue not yet mentioned lies in the detection of time-variant couplings. Usually, such an analysis can be easily done using moving window techniques. This reduces the station- 
arity requirement for the whole time series, to stationarity inside the window. However, the time scale on which couplings can be identified is strongly limited by the amount of data points needed to employ a certain coupling measure. Thus, it is nearly impossible to analyse transient events, which might change the coupling structure for very short periods of time, as compared to typical time scales on which changes occur in a given system. One way to circumvent this problem is to make sure the measured data has been recorded with a sufficient sampling rate. Depending on the data this is not always the case. Additionally, even if there is a sufficient time resolution, the stationarity requirement is usually not met during transients. How to solve this problem and how to extend common coupling measures to cope transient changes in the coupling structure using an ensemble approach, is the main subject of this work. In chapter 2 the ensemble approach will be introduced and explained. Also selected coupling measures will be presented together with a description on how to extend these measures using said approach. The following chapters 3,4 , and 5 contains different models to test the new extended coupling measures, as well as the results of the tests. Also, applications to real world systems are shown. The results will then be discussed and interpreted in the last chapter 6, where also an outlook for future applications and developments will be given. 


\section{Methods}

In this chapter, to overcome the problem of stationarity requirements of many coupling measures, paired with the wish to be able to analyse time-variant couplings on short time scales mentioned in the previous chapter, an ensemble approach to enhance existing coupling measures for a dynamic coupling analysis with a very high temporal solution is introduced and explained. Several coupling analysis tools working well also for short time series are then described, highlighting their strengths and limitations. In this section also the permutation symbolic coupling traces are introduced for the first time. Finally, all methods presented will be extended by applying the aforementioned ensemble approach. Thus, in this work the existing applications of the ensemble idea will be expanded to Granger causality and symbolic dynamics.

\subsection{Ensemble approach}

The detection of time-variant coupling structures is an important research issue, since many systems from fields encompassing physics, physiology, neuroscience, chemistry, biology, climate research, economy, etc. display dynamic changes in the system structure. These changes might be based on internal or external disturbances, like for example shocks or crises in economy [Zan09], large-scale events (e.g. El Niño or volcanic events) in climate research [MBMK12, RDR $\left.{ }^{+} 13\right]$, event-related potentials in neuroscience [CTK78], and sleep apnoea in physiology [LB01], or on inherent transitions between different regimes, like changes of sleep stages [IAICQ07], or seasons in the climate. Often, the time periods before and after such a transition are analysed in order to study differences in dynamic behaviour, coupling structure, etc., but the transition itself is regarded as an undesirable complication. This is because it usually happens on a much shorter time scale than adequately resolved by the data on hand and generally destroys any stationarity assumptions. Thus, also a windowed analysis approach would not work.

In order to overcome this problem, methods based on multiple realisations of a given process have been developed to e.g. detect transient chaos [DLK01, JT94], to denoise transient signals [ELS ${ }^{+}$00, SL09b], and also to characterise couplings [ALD06, IOLK08b, $\mathrm{KES}^{+}$04, $\mathrm{KTR}^{+}$08, LW08, MKWL11, WFL10]. The idea bears resemblance to the ergodic theorem of thermodynamics [Bir31] where a time average of one particle can be exchanged for a space average of an ensemble of particles at one time point. So, instead of estimating a given coupling measure over a time period, the averaging process is conducted across an ensemble of multiple realisations of the time series in question (see fig. 2.1). The ensemble could either be built by repeatedly performing a measurement of the same experiment on possibly several subjects, like for ex- 
ample an orthostatic test $\left[\mathrm{BKO}^{+} 08, \mathrm{BOL}^{+} 07\right]$ (head-up tilt or standing up after lying down for an elongated period of time), or by using inherent repeating events in a single time series, like several apnoea (cessation of airflow) during sleep [CRA $\left.{ }^{+} 14, \mathrm{GRS}^{+} 11, \mathrm{LB}^{2} 1, \mathrm{PRG}^{+} 12\right]$, [6]. This approach is theoretically applicable to almost all existing coupling measures, of course keeping in mind the requirements and limitations of the respective methods.

After it has been built, it is important to time rectify the ensemble. This can usually be done by aligning the individual ensemble members by means of a synchronisation point $T_{0}$, e.g. the beginning of the event regarded (see fig. 2.1). Corrections can be done by slightly shifting the ensemble measures against each other and looking for the shifting parameter where a maximum correlation can be achieved. Next, the respective coupling measures can be computed

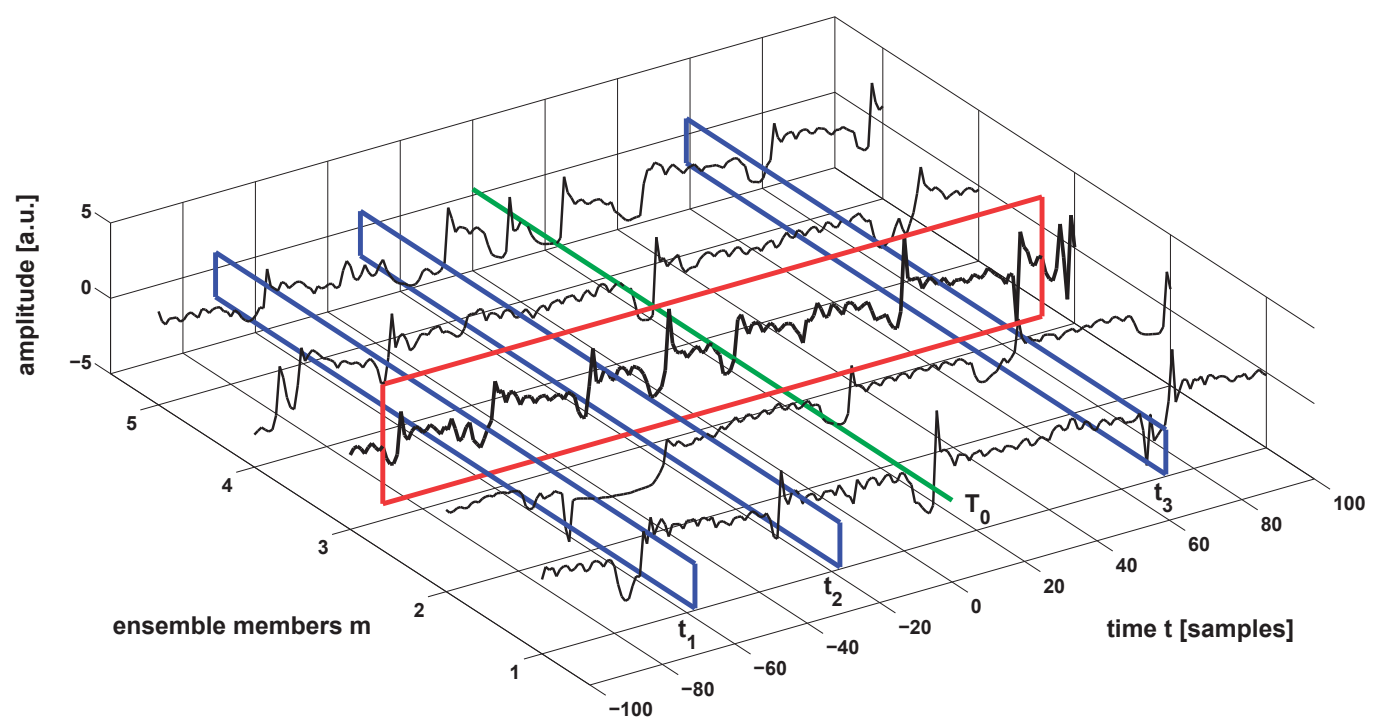

Figure 2.1: This figure shows the concept of time averages (red box) vs. ensemble averages (blue boxes). The time point $T_{0}$ (green line) signifies the synchronisation point at which the ensemble members are aligned. The other points $t_{1}, t_{2}$, and $t_{3}$ just represent possible times at which the ensemble average is performed.

by substituting the time average by the ensemble average. For the measures described in the remainder of this chapter, except Granger causality, the results are presented in a graph where the time lag $\tau$ is plotted over the time $t$. A colour coding represents the coupling strength and, if applicable, symmetric or diametric behaviour, respectively, while the sign of the time lag determines the coupling direction $(\tau<0$ for the first variable driving the second) (see fig. $2.2)$. 


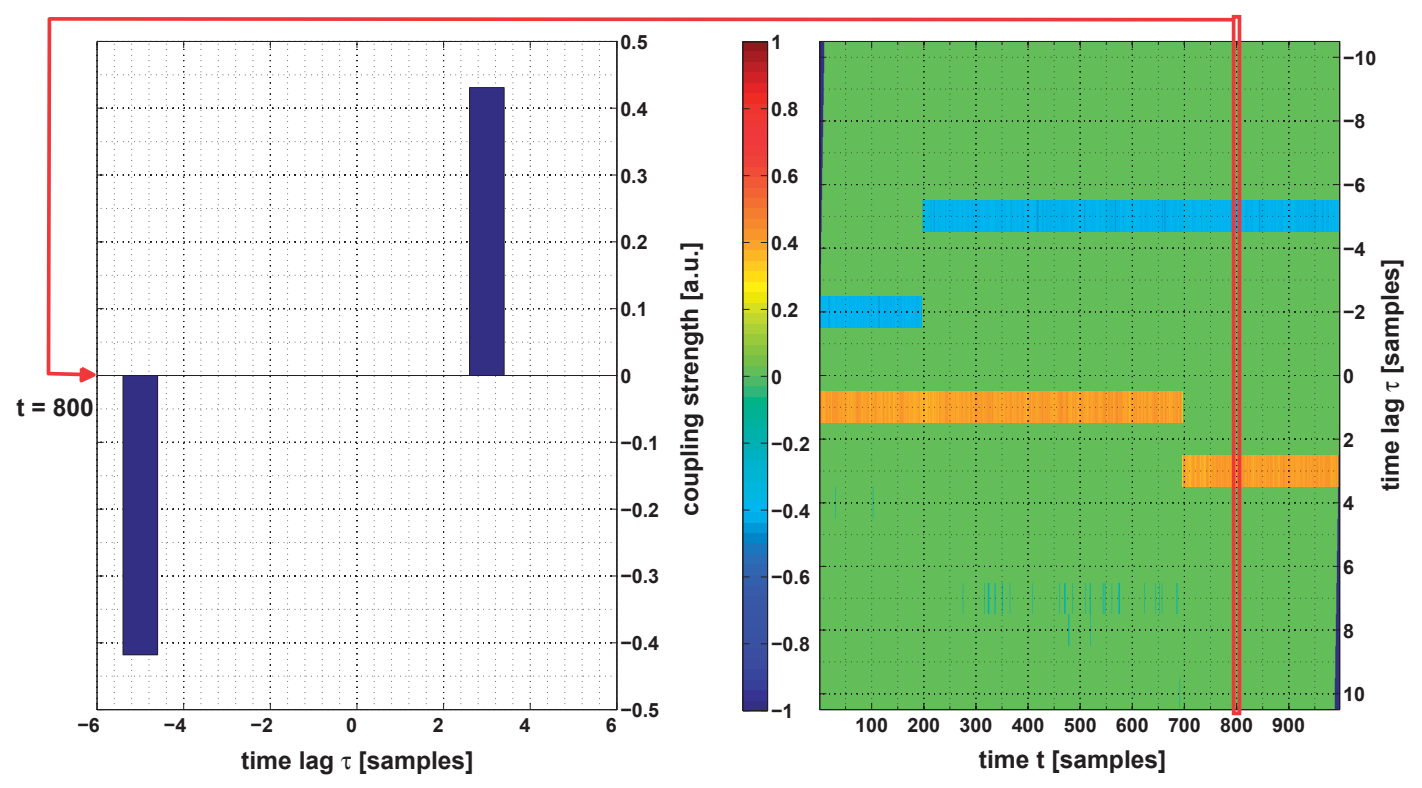

Figure 2.2: This figure explains how to read the resulting graphs for the bivariate time resolved coupling measures presented in this work. On the left, a graph shows a result displaying the coupling strength at the according time lags $\tau$ for one time point (here $t=800$ ). This representation is identical to the one one would receive, when using the classical time average approach. On the right, the coupling strength for each time lag $\tau$ and each time point $t$ is colour coded. Depending on the coupling measure used, positive (red) and negative (blue) values might occur, depicting symmetric or diametric behaviour, respectively.

The time resolution to be expected with the ensemble extension depends on the coupling measures used, since the estimations often are done over a short range of time points. The expected resolution will be given for each measure regarded in this work. To determine the significance of the results obtained using this approach, a surrogate method would appear to be ideally suited at a first glance, since we do not have to rely on known methods like random, Fourier transform, permutation, or twin surrogates [VP08]. Instead, the regarded time series $x^{(m)}(t)$ and $y^{(m)}(t)$ ( $m$ is the ensemble index) can be randomly paired with other members from the ensemble, e.g. $x^{(m)}(t)$ and $y^{(k)}(t)$, where $m \neq k$. Theoretically, this should break up any couplings between the time series, thus providing the desired surrogates. However, this only holds true during stationary phases. Of course, the transient events regarded, display identical behaviour in the time series. Therefore, any couplings found during the transients would be deemed not significant, since all surrogates show the same behaviour in this case. Hence, to test significance in these cases either empirical or quantile (e.g. only values greater than $95 \%$ of the coupling strength values over all time lags $\tau$ ) tests have to be applied. 
In this work the applications of the ensemble approach will be extended to Granger causality and to symbolic dynamics, thus significantly enlarging the set of tools for the analysis of timedependent couplings during transient behaviour.

\subsection{Classical measures}

In this section two well-known measures from time series analysis not inherently associated with the detection of coupling directions are presented. It is shown how to adapt these measures for a coupling analysis and how to extend them using the ensemble approach.

\subsubsection{Correlation}

One of the simplest bivariate coupling measures is based on the so-called Pearson correlation $\rho_{X Y}$ [Gal86, Pea95], which was developed to quantify the magnitude of linear interrelation between two time series $x(t)$ and $y(t)$. It is given by

$$
\rho_{X Y}=\frac{\operatorname{Cov}(x(t), y(t))}{\sqrt{\operatorname{Var}(x(t)) \operatorname{Var}(y(t))}},
$$

where $X$ and $Y$ are the two processes regarded, $\operatorname{Cov}()$ and $\operatorname{Var}()$ describe the covariance and the variance, respectively. The value of $\rho_{X Y}$ lies between $\rho_{X Y}=1$, total positive correlation, and $\rho_{X Y}=-1$, total negative correlation, while $\rho_{X Y}=0$ means no correlation. To infer information about possible causal structures, a time lag $\tau$ between the time series can be introduced, resulting in the so-called cross-correlation

$$
\rho_{X Y}(\tau)=\frac{\operatorname{Cov}(x(t), y(t+\tau))}{\sqrt{\operatorname{Var}(x(t)) \operatorname{Var}(y(t))}} .
$$

Depending on for which choice of $\tau$ the value $\left|\rho_{X Y}(\tau)\right|$ is highest, one can draw conclusions about the predominant coupling structure (e.g. $\tau<0$ means $Y$ drives $X$ and vice versa).

\subsubsection{Mutual information}

Another way to characterise the connection between two random processes $X$ and $Y$ is given by the so-called mutual information $I_{X Y}$ [Sha48]. It describes the difference between the entropy $H(X, Y)$ of the joint processes and the sum of the single entropies $H(X)$ and $H(Y)$ via

$$
I_{X Y}=H(X)+H(Y)-H(X, Y) .
$$


The entropies are computed as

$$
H(X)=-\int_{x} p(x) \log p(x) d x
$$

and

$$
H(X, Y)=-\int_{x} \int_{y} p(x, y) \log p(x, y) d x d y,
$$

which in the end leads to

$$
\begin{aligned}
I_{X Y} & =-\int_{x} p(x) \log p(x) d x-\int_{y} p(y) \log p(y) d y+\int_{x} \int_{y} p(x, y) \log p(x, y) d x d y \\
& =\int_{x} \int_{y} p(x, y) \log \frac{p(x, y)}{p(x) p(y)} d x d y .
\end{aligned}
$$

The advantage of this measure compared to the Pearson correlation lies in the ability of the mutual information to also detect nonlinear dependencies. But, to estimate the probability distributions in order to compute the necessary entropies, usually a bigger amount of data is needed.

To transform the simple mutual information into a measure to assess the coupling structure of two systems, the easiest way is again to introduce a time lag $\tau$ between the time series. The lagged mutual information is given by

$$
I_{X Y}(\tau)=\int p(x(t), y(t+\tau)) \log \frac{p(x(t), y(t+\tau))}{p(x(t)) p(y(t+\tau))} d x d y
$$

Again the coupling structure can be determined by the value of $I_{X Y}(\tau)$ and the value of $\tau$ itself. For most practical applications, the probability distribution $p$ can be estimated using a suitable histogram method. The intgrals would then be replaced by sums.

\subsubsection{Ensemble cross-correlation (EXCORR) and ensemble mutual information (EMI)}

The extension of cross-correlation and mutual information using the ensemble approach is pretty straightforward. The time dependent lagged ensemble cross-correlation is given by

$$
\rho_{X Y}^{(m)}(t, \tau)=\frac{\operatorname{Cov}\left(x^{(m)}(t), y^{(m)}(t+\tau)\right)}{\sqrt{\operatorname{Var}\left(x^{(m)}(t)\right) \operatorname{Var}\left(y^{(m)}(t)\right)}} .
$$

In this case $t$ stands for a fixed point in time and $m$ describes the average across the ensemble instead of over the time $t$. 
Analogously, the time dependent lagged mutual information can be determined by

$$
I_{X Y}^{(m)}(t, \tau)=\sum p\left(x^{(m)}(t), y^{(m)}(t+\tau)\right) \log \frac{p\left(x^{(m)}(t), y^{(m)}(t+\tau)\right)}{p\left(x^{(m)}(t)\right) p\left(y^{(m)}(t+\tau)\right)} .
$$

Again $t$ represents a fixed time point instead of the average over all time points while $m$ depicts the ensemble average.

Since both of the measures do not rely on any estimation across several time points, the expected time resolution of the ensemble cross-correlation and the ensemble mutual information is of the same order as the resolution the data has been recorded with.

\subsection{Granger causality}

\subsubsection{Classical Granger causality}

In the year 1963 C. W. J. Granger [Gra63] proposed a method to estimate causal relationships between two time series. He extended this method later in 1969 [Gra69] which is known today as Granger causality. The basic principle of this framework is grounded on the assumption, that a significantly improved prediction of future values of a time series $x_{2}(t)$, if the past of another time series $x_{1}(t)$ is known additionally to the past of $x_{2}(t)$ itself, $(t=1, \ldots, N)$, indicates a dynamic influence from the system $X_{1}$ onto the system $X_{2}$. Of course, Granger causality and causality in the common meaning have to be distinguished. Granger causality just tells us something about predictability. Nonetheless, it is still useful when building descriptive models and trying to get an idea of the interconnections between smaller subsystems in a bigger complex system. To upkeep at least the possibility of true causality one has to keep in mind the time points used for the prediction: using for example values like $x_{1}(t+\tau)$ for the prediction of $x_{2}(t)$ could well point to Granger causality but surely cannot indicate true causality as the time order plays a crucial role.

Granger proposed the use of AR-models to represent the time series. This approach is still often used today. Here, a bivariate AR-process of order $\Omega$ will be fitted to the two time series in question

$$
\begin{aligned}
& x_{1}^{(r)}(t)=\sum_{\tau=1}^{\Omega} a_{11}^{(r)}(\tau) x_{1}(t-\tau)+\epsilon_{1}^{(r)}(t), \\
& x_{2}^{(r)}(t)=\sum_{\tau=1}^{\Omega} a_{22}^{(r)}(\tau) x_{2}(t-\tau)+\epsilon_{2}^{(r)}(t) .
\end{aligned}
$$

The variables $\epsilon_{1}^{(r)}$ and $\epsilon_{2}^{(r)}$ describe independent white noise, and $a_{11}^{(r)}(\tau)$ and $a_{22}^{(r)}(\tau)$ denote the 
coefficients to be estimated using for example a least squares method. The superscript index $(r)$ denotes the restricted model, i.e. the model using not all available information.

This whole process is then repeated using the information from the second time series

$$
\begin{aligned}
& x_{1}^{(u)}(t)=\sum_{\tau=1}^{\Omega} a_{11}^{(u)}(\tau) x_{1}(t-\tau)+\sum_{\tau=0,1}^{\Omega} a_{21}^{(u)}(\tau) x_{2}(t-\tau)+\epsilon_{1}^{(u)}(t), \\
& x_{2}^{(u)}(t)=\sum_{\tau=0,1}^{\Omega} a_{12}^{(u)}(\tau) x_{1}(t-\tau)+\sum_{\tau=1}^{\Omega} a_{22}^{(u)}(\tau) x_{2}(t-\tau)+\epsilon_{2}^{(u)}(t) .
\end{aligned}
$$

The superscript index $(u)$ describes the unrestricted model using all available variables.

Whether the summation index $\tau$ when incorporating the second time series starts at $\tau=0$ or $\tau=1$ depends on the data used. On the one hand, to at least maintain the temporal order between the possible cause and effect one should start with $\tau=1$. Thus one can assure that influences from the second series really come from the past. On the other hand, when measuring data there always is some averaging involved. Depending on the sampling rate and the time scale on which a potential information transfer might occur one could argue to include $\tau=0$. As an example, regard a climate time series of mean monthly temperatures. Processes influenced by the temperature can easily be assumed to work on time scales of weeks or days. In this case one should consider also employing the lag $\tau=0$ values.

To determine the optimum model order $\Omega$ before calculating Granger causality, there are several possibilities. The most common ones are the Akaike information criterion (AIC) [Aka69] and the Bayesian information criterion (BIC) [Sch78]. Both functions compare the computational effort of a given model with its performance according to

$$
\operatorname{AIC}(\Omega)=\log (\operatorname{det} \Sigma)+\frac{2 n_{v a r}^{2} \Omega}{N}
$$

for the Akaike criterion and

$$
\operatorname{BIC}(\Omega)=\log (\operatorname{det} \Sigma)+\log (N) \frac{n_{v a r}^{2} \Omega}{N},
$$

for the Bayesian variant. Here, $\Sigma$ is the covariance matrix of the residuals $\varepsilon$ from the multivariate regression, $n_{v a r}$ is the number of variables used, $\Omega$ is the model order, and $N$ is the length of the data series regarded. The minimum of the AIC and BIC values gives the model order for the best compromise between cost and efficiency. Which of these methods should be chosen to determine the best model order depends on several questions [ADP14] like whether the best prediction (AIC) or finding the correct model (BIC) is more important.

After having fitted the respective AR-models the comparison of the variances of the error 
terms $\varepsilon_{1}^{(r)}=x_{1}(t)-x_{1}^{(r)}(t)$ and $\varepsilon_{2}^{(r)}=x_{2}(t)-x_{2}^{(r)}(t)$ with $\varepsilon_{1}^{(u)}$ and $\varepsilon_{2}^{(u)}$, respectively, gives the information about the predominant coupling direction. If, for example, $\operatorname{Var}\left(\varepsilon_{2}^{(u)}\right)<\operatorname{Var}\left(\varepsilon_{2}^{(r)}\right)$ and $\operatorname{Var}\left(\varepsilon_{1}^{(u)}\right) \approx \operatorname{Var}\left(\varepsilon_{1}^{(r)}\right)$ holds true, the influence from the process $X_{1}$ on the process $X_{2}$ would be greater than the other way around. In this case $X_{1}$ Granger causes $X_{2}$.

To test the results from Granger's method for significance the so-called Granger-Sargent (GS) test [HSPVB07]

$$
G S_{X_{1} \rightarrow X_{2}}=\frac{\sum_{i}\left(\varepsilon_{2}^{(r)}\right)^{2}-\sum_{i}\left(\varepsilon_{2}^{(u)}\right)^{2}}{\sum_{i}\left(\varepsilon_{2}^{(u)}\right)^{2}} \frac{N-\left(n_{v a r}+1\right) \Omega}{\Omega} .
$$

can be used. The $G S$ test statistics follow an $F$-distribution with $\Omega$ and $N-\left(n_{v a r}+1\right) \Omega$ degrees of freedom allowing for an easy determination of the critical values belonging to the desired significance level.

\subsubsection{Conditional Granger causality}

The classical Granger causality is only suited for bivariate systems and thus cannot detect indirect couplings. For example the method could indicate a coupling between subsystems $X_{1}$ and $X_{2}$ while in reality both systems are driven by a third one $X_{3}$. If this third system is known and can be measured, the classical Granger causality can easily be extended to a multivariate tool according to [Gew84, ZF09], usually known by the term conditional Granger causality. Therefore, an AR-model using all available variables is fitted for each variable. Additionally, another AR-model using all variables but the one $\left(X_{k}\right)$, whose causal influence we are interested in, is determined. For a system with $n_{\text {var }}$ variables and $j, k \in\left\{1, \ldots, n_{\text {var }}\right\}$ the equations

$$
\begin{aligned}
& x_{k j}^{(r)}(t)=\sum_{i=1 ; i \neq k}^{n_{\text {var }}} \sum_{\tau=0 ; 1}^{\Omega} a_{i j}^{(r)}(\tau) x_{i}(t-\tau)+\epsilon_{k j}^{(r)}(t), \\
& x_{j}^{(u)}(t)=\sum_{i=1}^{n_{\text {var }}} \sum_{\tau=0 ; 1}^{\Omega} a_{i j}^{(u)}(\tau) x_{i}(t-\tau)+\epsilon_{j}^{(u)}(t)
\end{aligned}
$$

let us determine the influence from $X_{k}$ to $X_{j}$ conditioned on $\left\{X_{i} ; i \notin\{j, k\}\right\}$ via the term

$$
F_{X_{k} \rightarrow X_{j} \mid\left\{X_{i} ; i \notin\{j, k\}\right\}}^{(c)}=\log \frac{\operatorname{Var}\left(\varepsilon_{k j}^{(r)}\right)}{\operatorname{Var}\left(\varepsilon_{j}^{(u)}\right)} .
$$


Now, if the system $X_{k}$ would give no new information about the system $X_{j}$, the variances of the error terms should be about equal and thus $F_{X_{k} \rightarrow X_{j} \mid\left\{X_{i} ; i \neq j, k\right\}}^{(c)} \approx 0$ should hold true and there actually would not be an influence from $X_{k}$ to $X_{j}$ in the sense of Granger causality, even though the classical method might have found one.

To allow for the detection of nonlinear couplings for at least a subset of all possible cases, without dramatically increasing the computational effort needed for the estimation of Granger causality, one can simply add a quadratic term to the AR-model. The resulting equations are

$$
x_{k j}^{(r, q)}(t)=\sum_{i=1 ; i \neq k}^{n_{\text {var }}} \sum_{\tau=0 ; 1}^{\Omega} a_{i j}^{(r)}(\tau, t) x_{i}(t-\tau)+a_{i j}^{(r, q)}(\tau, t)\left(x_{i}(t-\tau)\right)^{2}+\epsilon_{k j}^{(r)}(t)
$$

and

$$
x_{j}^{(u, q)}(t)=\sum_{i=1}^{n_{\text {var }}} \sum_{\tau=0 ; 1}^{\Omega} a_{i j}^{(u)}(\tau, t) x_{i}(t-\tau)+a_{i j}^{(u, q)}(\tau, t)\left(x_{i}(t-\tau)\right)^{2}+\epsilon_{j}^{(u)}(t) .
$$

The superscript $q$ describes the adding of the quadratic terms. To further decrease the computational needs, the regarded time lags for the quadratic terms can be restricted to only a few or even just one value of $\tau$.

\subsubsection{Partial Granger causality}

One problem remaining with conditional Granger causality is its inability to account for latent variables, i.e. variables we do not know about. An approach to at least partially account for such variables is given by the partial Granger causality [ $\left.\mathrm{GSK}^{+} 08\right]$, an extension to the conditional Granger causality. The method is based on the assumption that influences of latent variables can be detected via the prediction errors of the model used, if the latent variables represent stationary processes. The noise terms $\epsilon_{k j}^{(r)}(t)$ and $\epsilon_{j}^{(u)}(t)$ from the models could under this assumption be decomposed into

$$
\epsilon_{k j}^{(r)}(t)=\epsilon_{k j}^{(r), \text { model }}(t)+\epsilon_{k j}^{(r), \text { latent }}(t)
$$

and

$$
\epsilon_{j}^{(u)}(t)=\epsilon_{j}^{(u), \text { model }}(t)+\epsilon_{j}^{(u), \text { latent }}(t)
$$

We are now interested in correlations between the two error terms $\varepsilon_{k j}^{(r)}$ and $\varepsilon_{j}^{(u)}$. Therefore, a variant of the well-known partial correlation is used. Hence, we regard the covariance matrices $\Sigma_{k j}^{(r)}$ and $\Sigma_{j}^{(u)}$ of the error terms for the restricted and the unrestricted model, respectively, 
when determining the influence of system $X_{k}$ onto system $X_{j}$ conditioned on all known systems $\left\{X_{i} ; i \notin\{j, k\}\right\}$. After eliminating the column and the row in $\Sigma_{j}^{(u)}$ containing the system $X_{k}$ to obtain $\Sigma_{k j}^{(u)}$ the covariance matrices are separated into four block matrices, giving

$$
\Sigma_{k j}^{(r)}=\left[\begin{array}{c|c}
\operatorname{Var}\left(\varepsilon_{k j}^{(r)}\right) & \operatorname{Cov}\left(\varepsilon_{k j}^{(r)},\left\{\varepsilon_{k i}^{(r)} ; i \notin\{j, k\}\right\}\right) \\
\hline \operatorname{Cov}\left(\left\{\varepsilon_{k i}^{(r)} ; i \notin\{j, k\}\right\}, \varepsilon_{k j}^{(r)}\right) & \operatorname{Cov}\left(\left\{\varepsilon_{k i}^{(r)} ; i \notin\{j, k\}\right\}\right)
\end{array}\right]=\left[\begin{array}{c|c}
\Sigma_{k j, 11}^{(r)} & \Sigma_{k j, 12}^{(r)} \\
\hline \Sigma_{k j, 21}^{(r)} & \Sigma_{k j, 22}^{(r)}
\end{array}\right]
$$

and

$$
\Sigma_{k j}^{(u)}=\left[\begin{array}{c|c}
\operatorname{Var}\left(\varepsilon_{j}^{(u)}\right) & \operatorname{Cov}\left(\varepsilon_{j}^{(u)},\left\{\varepsilon_{i}^{(u)} ; i \notin\{j, k\}\right\}\right) \\
\hline \operatorname{Cov}\left(\left\{\varepsilon_{i}^{(u)} ; i \notin\{j, k\}\right\}, \varepsilon_{j}^{(u)}\right) & \operatorname{Cov}\left(\left\{\varepsilon_{i}^{(u)} ; i \notin\{j, k\}\right\}\right)
\end{array}\right]=\left[\begin{array}{c|c}
\Sigma_{k j, 11}^{(u)} & \Sigma_{k j, 12}^{(u)} \\
\hline \Sigma_{k j, 21}^{(u)} & \Sigma_{k j, 22}^{(u)}
\end{array}\right]
$$

Following the partial correlation further yields the corrected variances of the error terms

$$
R_{k j}^{(r)}=\Sigma_{k j, 11}^{(r)}-\Sigma_{k j, 12}^{(r)}\left(\Sigma_{k j, 22}^{(r)}\right)^{-1} \Sigma_{k j, 21}^{(r)}
$$

and

$$
R_{k j}^{(u)}=\Sigma_{k j, 11}^{(u)}-\Sigma_{k j, 12}^{(u)}\left(\Sigma_{k j, 22}^{(u)}\right)^{-1} \Sigma_{k j, 21}^{(u)}
$$

with again $i, j, k \in\left\{1, \ldots, n_{v a r}\right\}$ and $n_{v a r}$ being the number of variables. Analogous to the conditional Granger causality, we can then define

$$
F_{X_{k} \rightarrow X_{j} \mid\left\{X_{i} ; i \notin\{j, k\}\right\}}^{(p)}=\log \frac{\operatorname{det}\left(R_{k j}^{(r)}\right)}{\operatorname{det}\left(R_{k j}^{(u)}\right)} .
$$

Using this measure $F_{X_{k} \rightarrow X_{j} \mid\left\{X_{i} ; i \notin\{j, k\}\right\}}^{(p)}$ it is now possible to quantify the influence of system $X_{k}$ onto $X_{j}$ conditioned on the remaining known variables $\left\{X_{i} ; i \notin\{j, k\}\right\}$ and other latent variables as long as they stem from stationary processes. The method of partial Granger causality has proved to be superior to the classical and conditional versions on numerous test cases and applications to neuronal data $\left[\mathrm{GSK}^{+} 08\right]$.

\subsubsection{Ensemble Granger causality (EGC)}

The extension of Granger causality for time-dependent analyses during transients using the ensemble approach further broadens the already vast field of possible applications of this versatile coupling measure. To expand the above presented versions of Granger causality using the ensemble approach, just the way in which the parameters for AR-models are computed, has to 
be changed. For a system with $n_{v a r}$ variables and $j, k \in\left\{1, \ldots, n_{v a r}\right\}$ the equations

$$
\begin{aligned}
x_{k j}^{(r),(m)}(t) & =\sum_{i=1 ; i \neq k}^{n_{\text {var }}} \sum_{\tau=0 ; 1}^{\Omega} a_{i j}^{(r),(m)}(\tau, t) x_{i}^{(m)}(t-\tau)+\epsilon_{k j}^{(r),(m)}(t), \\
x_{j}^{(u),(m)}(t) & =\sum_{i=1}^{n_{\text {var }}} \sum_{\tau=0 ; 1}^{\Omega} a_{i j}^{(u),(m)}(\tau, t) x_{i}^{(m)}(t-\tau)+\epsilon_{j}^{(u),(m)}(t)
\end{aligned}
$$

let us determine the influence from $X_{k}$ to $X_{j}$ conditioned on $\left\{X_{i} ; i \notin\{j, k\}\right\}$. The only differences to equations (2.1) and (2.2) lie in the way the regression is performed and the meaning of the time index $t$. Here, the estimation of the parameters $a^{(r),(m)}$ and $a^{(u),(m)}$ is carried out across the ensemble and $t$ means a fixed time point instead of the average over all time points, i.e. $a^{(r),(m)}$ and $a^{(u),(m)}$ are now time-dependent. The starting value of $\tau$ with either $\tau=0$ or $\tau=1$ depends on whether internal or external influences are regarded. Since the coupling direction is identified using only the error terms from the model, the same index as before can be utilised

$$
F_{X_{k} \rightarrow X_{j} \mid\left\{X_{i} ; i \notin\{j, k\}\right\}}^{(c),(m)}(t)=\log \frac{\operatorname{Var}\left(\varepsilon_{k j}^{(r),(m)}(t)\right)}{\operatorname{Var}\left(\varepsilon_{j}^{(u),(m)}(t)\right)},
$$

which is now of course also time dependent.

This approach can also be extended by using the nonlinear terms as presented in subsection 2.3.2. The resulting equations are

$$
x_{k j}^{(r, q),(m)}(t)=\sum_{i=1 ; i \neq k}^{n_{\text {var }}} \sum_{\tau=0 ; 1}^{\Omega} a_{i j}^{(r),(m)}(\tau, t) x_{i}^{(m)}(t-\tau)+a_{i j}^{(r, q),(m)}(\tau, t)\left(x_{i}^{(m)}(t-\tau)\right)^{2}+\epsilon_{k j}^{(r),(m)}(t)
$$

and

$$
x_{j}^{(u, q),(m)}(t)=\sum_{i=1}^{n_{\text {var }}} \sum_{\tau=0 ; 1}^{\Omega} a_{i j}^{(u),(m)}(\tau, t) x_{i}^{(m)}(t-\tau)+a_{i j}^{(u, q),(m)}(\tau, t)\left(x_{i}^{(m)}(t-\tau)\right)^{2}+\epsilon_{j}^{(u),(m)}(t) .
$$

The superscript $q$ describes again the adding of the quadratic terms. The time resolution achieved by the ensemble Granger causality is of the same level as the choice of the model order $\Omega$. For the remainder of this work only the ensemble partial Granger causality will be used. Whenever the talk is about Granger causality from now on, actually this method is meant. The code for this version of Granger causality for MATLAB (MATLAB and Statistics Toolbox Release 2012a, The MathWorks, Inc., Natick, Massachusetts, United States) can be found at http://tocsy.pik-potsdam.de/coupling.php. 


\subsection{Symbolic coupling traces}

\subsubsection{Classic symbolic coupling traces}

The symbolic coupling traces (SCT) were introduced by Wessel et al. [WSR ${ }^{+} 09$ ] and are an extension of a bivariate joint symbolic dynamics method $\left[\mathrm{BWH}^{+} 02\right]$ which was developed to characterise and interpret the complex and highly nonlinear interactions between heart rate and systolic blood pressure. For both methods a dynamical system represented by two onedimensional time series $x(t)$ and $y(t)$ is considered, which are then transformed into coarse grained symbolic time series $s_{x}(t)$ and $s_{y}(t)$ according to

$$
s_{z}(t)= \begin{cases}1, & z(t) \leq z(t+\vartheta) \\ 0, & z(t)>z(t+\vartheta)\end{cases}
$$

The time lag $\vartheta$ is usually set to $\vartheta=1$ but can also be chosen as another number of time steps in order to accommodate a priori knowledge about the time scales on which the couplings act. These symbol series in turn are used to construct series of words $w_{z}(t)$ where each word contains $l$ successive symbols (see table 2.1). Because of the binary alphabet in this case, this gives $d=2^{l}$ different possibilities of words. Larger values of $\vartheta$ work like an averaging process across the area defined by $\vartheta$ and $l$.

$$
\begin{aligned}
& \begin{array}{lllllllllll}
x(t)= & \ldots & 8 & 6 & 9 & 11 & 12 & 8 & 13 & 5 & \ldots
\end{array} \\
& y(t)=\begin{array}{llllllllll}
\frac{11}{3} & 7 & 2 & 5 & 11 & 10 & 6 & \ldots
\end{array} \\
& s_{x}(t)=\quad \ldots \quad 0 \quad 11 \quad \begin{array}{llllllll}
1 & 1 & 0 & 1 & 0 & \ldots
\end{array}
\end{aligned}
$$

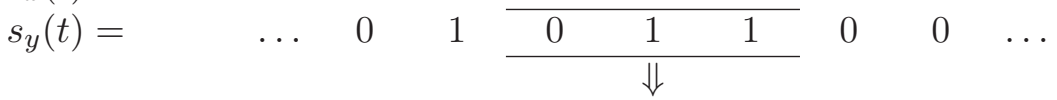

$$
\begin{aligned}
& w_{x}(t)=\quad \ldots \quad 011 \quad 111 \quad 110 \quad 101 \quad 010 \quad \ldots
\end{aligned}
$$

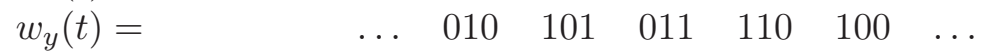

Table 2.1: This scheme shows how to transform time series $x(t)$ and $y(t)$ into word sequences $w_{x}(t)$ and $w_{y}(t)$ with $l=3$ via the symbol series $s_{x}(t)$ and $s_{y}(t)$, respectively.

From the word sequences generated in this way for time series $x(t)$ and $y(t)$, a bivariate word distribution can now be estimated as

$$
\Pi_{i j}=P\left(w_{x}(t)=W_{i}, w_{y}(t)=W_{j}\right)
$$

Here, $W_{i}$ and $W_{j}$ denote certain words out of the whole vocabulary of $d=2^{l}$ different words and $\Pi_{i j}$ is the joint probability of words $W_{i}$ and $W_{j}$ appearing at the same time $t$ in the word series $w_{x}$ and $w_{y}$, estimated over all values of $t$. To later be able to determine the coupling 
direction and the occurring lags, a time lag $\tau$ between the two word sequences $w_{x}$ and $w_{y}$ is introduced, resulting in the matrix

$$
(\Pi(\tau))_{i j}=P\left(w_{x}(t)=W_{i}, w_{y}(t+\tau)=W_{j}\right)
$$

One way to characterise this matrix could be to regard the joint Shannon entropy [Sha48] for each lag $\tau$. However, studies in [WSR ${ }^{+}$09] showed, that using Shannon entropy does not clearly reveal the correct time lags. Instead, the results improve a lot, when regarding only the difference between the occurrences of symmetric (e.g. $\left.w_{x}(t)=w_{y}(t+\tau)\right)$ and diametric words (e.g. $w_{x}(t)=$ '111' and $w_{y}(t+\tau)=$ '000'). The symmetric word frequency is represented by

$$
T(\tau)=\operatorname{Tr}(\Pi(\tau))=\sum_{i=j}(\Pi(\tau))_{i j}
$$

and the diametric word frequency by

$$
\bar{T}(\tau)=\sum_{i=1, \ldots, d ; j=d+1-i}(\Pi(\tau))_{i j}
$$

where $\operatorname{Tr}(\Pi(\tau))$ is the trace of the matrix $\Pi(\tau)$ and $d=2^{l}$ is the number of the possible different words. The difference $\Delta T=T-\bar{T}$ has proved to be an effective parameter to identify the coupling structure of bivariate systems. To assess the significance of the results thus obtained, an empiric test based on a simulation with bivariate white noise for different signal lengths has been developed $\left[\mathrm{SRM}^{+} 10\right]$. For the significance level $\alpha=0.01$ the critical values of $\Delta T$ are given as

$$
\Delta T_{\text {crit }}(N)= \pm 2.7005 \cdot N^{-0.5179}
$$

where $N$ is the number of data points regarded. Now, the coupling direction can be determined via the occurring time lags $\tau$ where $\Delta T$ is significant. The coupling strength is related to $|\Delta T|$ and $\operatorname{sgn}(\Delta T)$ tells us whether symmetric or diametric behaviour is dominant. Further insight into the systems in question might be gained by looking at the results of the SCT when using the absolute value of the time series as input.

Although the SCT are only a bivariate method, the conclusions drawn by using them are well suited to build descriptive models of the systems regarded. An extension for three or more systems is unfeasible, because of the curse of dimensionality [Bel57]. The occupation numbers in the resulting $n$-dimensional word distribution matrix for $n$ systems would get too small too fast. 


\subsubsection{Permutation symbolic coupling traces}

In 2002 Bandt and Pompe introduced a complexity measure for time series, termed permutation entropy, which combines concepts from entropy and symbolic dynamics [BP02]. This measure proved to be simple yet robust while taking the temporal order of the values in a given time series into account, a point usually neglected by other complexity measures like Shannon [Sha48] or Kolmogorov-Sinai entropy [KS03]. Permutation entropy essentially measures information based on the occurrence of permutation patterns built from the ranks of the values in a time series. In this thesis, the idea by Bandt and Pompe is modified to apply it to the symbolic coupling traces. Instead of the binary alphabet and the following words sequences, permutation patterns $\pi_{j}$ of length $l(j=1, \ldots, l !)$ are used. At first, sections of the time series $x(t)$ and $y(t)$ are converted into rank sequences $r_{x_{t}}$ and $r_{y_{t}}$ of length $l$, which in turn are matched with one of the possible permutation patterns $\pi_{j}$ (compare eq. (2.5) and fig. 2.3). If we encounter equal values in one rank sequence, there are different possibilities [8]. Here, the ranks are assigned according to the temporal order in which the equal values occur.
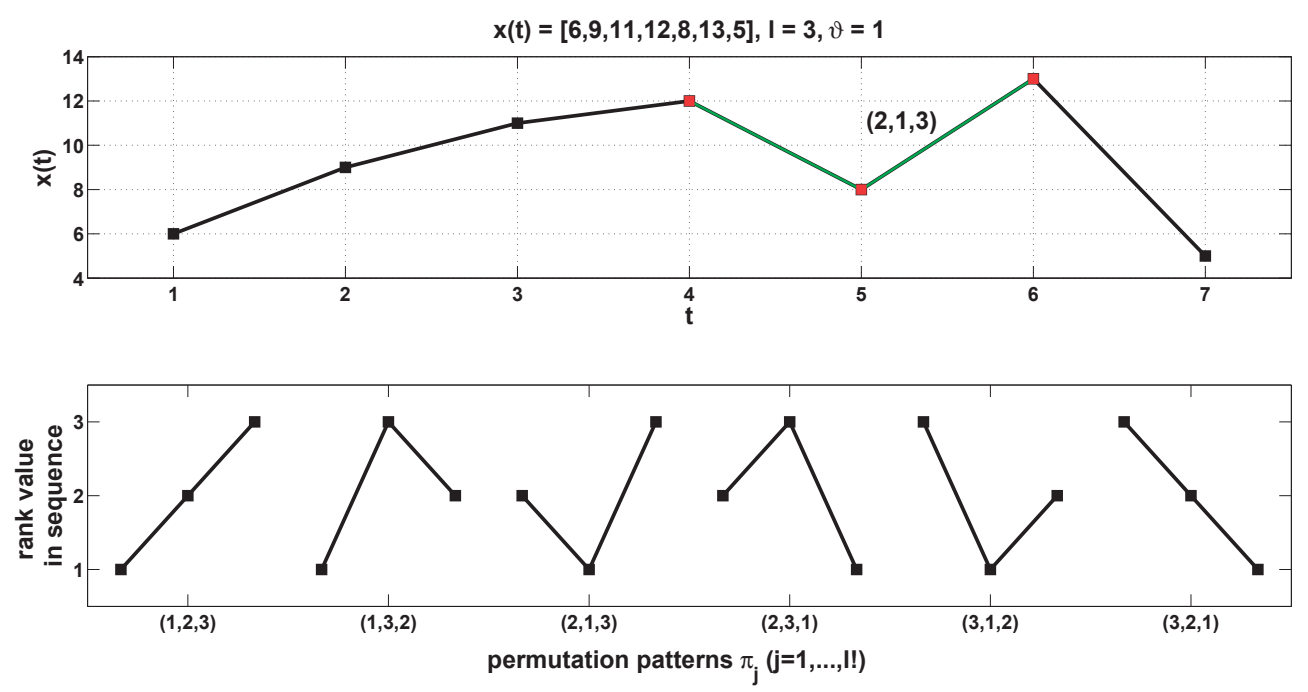

Figure 2.3: This figure shows an example of how to build the rank sequences from a given time series $x(t)$ with $\vartheta=1$ and $l=3$. Below, the different possible permutation patterns are presented. 


$$
\begin{aligned}
x(t), x(t+\vartheta), \ldots, x(t+(l-1) \vartheta) & \rightarrow r_{x_{t}}(1), \ldots, r_{x_{t}}(l) \rightarrow \pi_{j} \\
y(t), y(t+\vartheta), \ldots, y(t+(l-1) \vartheta) & \rightarrow r_{y_{t}}(1), \ldots, r_{y_{t}}(l) \rightarrow \pi_{j}
\end{aligned}
$$

Following the steps from the SCT, we estimate the probability distribution of the permutation patterns. To characterise the coupling structure again a time lag $\tau$ between the two time series regarded is introduced. As entropy measures also do not give satisfying results when characterising the probability distribution matrices, the same approach via the symmetric and diametric patterns is used and $\Delta T_{P}=T_{P}-\bar{T}_{P}$ is defined analogously to the classical SCT (see equations (2.3) and (2.4)). The subscript $P$ here just refers to the permutation approach. The difference between the classical and the permutation approach lies in the number of possible patterns regarded, which is higher in the permutation pattern case. This leads to lower occupation numbers for each combination, resulting in the best case in fewer falsely positive recognised coupling lags. Here also, the use of the absolute value of the time series might lead to further results.

\subsubsection{Ensemble symbolic coupling traces (ESCT and PESCT)}

In this subsection, the tools of symbolic coupling traces and permutation symbolic coupling traces will be extended using the ensemble approach. This opens the field of symbolic dynamics to be employed for coupling analysis of transient and nonstationary events.

Since the ensemble approach for the symbolic coupling traces takes only hold after the word sequences $w_{x}^{(m)}(t)$ and $w_{y}^{(m)}(t)$ or $r_{x t}^{(m)}$ and $r_{y_{t}}^{(m)}$ in the case of the permutation approach have been built for the whole ensemble (index $m$ ), the following steps are the same for both methods and the sequences $w^{(m)}$ and $r^{(m)}$ are used synonymously.

When estimating the probability distribution of the word occurrences, the histogram is now computed over the whole ensemble resulting in the time dependent matrix

$$
\left(\Pi^{(m)}(t, \tau)\right)_{i j}=P\left(w_{x}^{(m)}(t)=W_{i}, w_{y}^{(m)}(t+\tau)=W_{j}\right) .
$$

The index $m$ here stands for averaging across the ensemble and $t$ represents a fixed point in time. In the end, the symmetric and diametric word frequencies are again given by

$$
T^{(m)}(t, \tau)=\operatorname{Tr}\left(\Pi^{(m)}(t, \tau)\right)=\sum_{i=j}\left(\Pi^{(m)}(t, \tau)\right)_{i j}
$$


and

$$
\bar{T}^{(m)}(t, \tau)=\sum_{i=1, \ldots, d ; j=d+1-i}\left(\Pi^{(m)}(t, \tau)\right)_{i j}
$$

Via $\Delta T^{(m)}(t, \tau)=T^{(m)}(t, \tau)-\bar{T}^{(m)}(t, \tau)$ the coupling structure can be determined as before. In this case the same empirical approach to assess the significance of the results should hold true. The choice of the word length determines the expected time resolution of these two methods. The code for these two methods for MATLAB (MATLAB and Statistics Toolbox Release 2012a, The MathWorks, Inc., Natick, Massachusetts, United States) can be found at http://tocsy.pik-potsdam.de/coupling.php. 


\section{Applications to models}

In this chapter, the methods developed in chapter 2 are tested on different theoretical models to assess their performance potential and their limitations. The huge class of data-driven ARmodels is commonly used to simulate and predict real-world systems. Therefore, the coupling measures will be applied to multivariate AR-models with unidirectional and feedback coupling. To test their performance in nonlinear systems, coupled chaotic maps and NAARX-models will be employed. Additionally, the measures' robustness against noise, their performance for smaller amounts of data, and the dependence on coupling strength will be evaluated.

To evaluate the significance of the results delivered by the different methods, two approaches are chosen. For Granger causality the built-in F-test is used and the obtained values for $F_{X_{k} \rightarrow X_{j} \mid\left\{X_{i} ; i \notin\{j, k\}\right\}}^{(p)}$ are multiplied by one (significant) or zero (not significant). For the two symbolic measures first the empiric test from $\left[\mathrm{SRM}^{+} 10\right]$ is used and additionally, since during the tests still a lot of spurious couplings were found, only the results with an absolute value above the $(1-\alpha)$-quantile are used. That same test is also applied for the correlation and the mutual information measures.

The model order $\Omega$ for the Granger causality is usually also taken as the maximum lag for the other measures. It is chosen as a compromise between the results of the AIC and the BIC, and taking a reasonably small value to allow for shorter data series, while still being large enough to encompass the a priori knowledge about the maximum lags appearing in the models.

\subsection{Analysis}

\subsubsection{AR-models}

The first class of models to be analysed is the class of AR-models. These are often employed to describe certain time-varying processes in different fields like economics, climate, and medicine, because of certain features AR-models offer. Using AR-models it is possible to choose the parameters in a way that the spectral density of the model approximates any given continuous spectral density with arbitrary precision (pp. 148-149 in [KN06]). The consistency of the parameters of such a model over time is a necessary condition for fitting the parameters by means of time averaging. However, in this case we are interested in time-dependent parameters.

A time-variant AR-process of order $\Omega$ is given by

$$
x(t)=\sum_{\tau=1}^{\omega} a(\tau, t) x(t-\tau)+\epsilon(t) .
$$


In this univariate case, values of $x$ only depend on the past of $x$ itself and an innovative term $\epsilon$, usually in the form of Gaussian white noise charaterised by an expected value of zero and a certain variance depicting the strength of the influence of the stochastically independent innovative term. The multivariate version of an AR-process with $n_{v a r}$ variables is given by

$$
x_{j}(t)=\sum_{i=1}^{n_{v a r}} \sum_{\tau=1}^{\omega} a_{i j}(\tau, t) x_{i}(t-\tau)+\epsilon_{j}(t), \quad j \in\left\{1, \ldots, n_{v a r}\right\} .
$$

The normally distributed $\epsilon_{j}$ are independent for each variable.

\section{AR-model with univariate and feedback coupling}

To test the methods presented in chapter 2 we regard two different scenarios of AR-models. The first one consists of five variables displaying univariate (from $x_{4}$ to $x_{5}$ ) and bivariate (feedback between $x_{1}$ and $x_{2}$ ) coupling as well as a common driver $\left(x_{3}\right)$. The coupling structure is timedependent, dividing the regarded time span into four epochs with different time lags, coupling strengths, and influence effects (symmetric or diametric). The standard deviation of the white noise innovative term $\epsilon$ is $\sigma=0.01$. All model parameters can be found in table 3.1. The structure is also given in figure 3.3 (a). The realisations has been generated using normally distributed initial values for the variables and then running the equations for 10000 iterations from which only the last $N=1000$ data points have been used for the analysis. The ensemble consists of $M=1000$ realisations, aligned with the beginnings and endings of each epoch (see table 3.1). The significance level for this test has been set to $\alpha=0.05$ for all methods. The model order has been determined by the AIC-method $(\Omega=10)$ and the BIC-method suggested $(\Omega=18)$. According to the strategy of preferring a small model order and taking into account a priori knowledge, the model order for Granger causality and the maximum lag for the other method has been set to $\Omega=10$.

In figure 3.1 the significant results from the five regarded methods for the coupling between $x_{1}$ and $x_{2}$ are shown. The coupling structure is correctly identified for all epochs by all measures, except the ensemble mutual information (see figure 3.1 (e)), although also the other measures except Granger causality show spurious couplings at some lags and time points as well as some gaps when the coupling strength is reduced in the fourth epoch. The significant results for the univariate coupling between $x_{4}$ and $x_{5}$ are shown in figure 3.2. Here, Granger causality (figure 3.2 (a)) does not identify the change in the coupling structure in epoch three, because there is no change in the coupling strength. Although the other measures all identify the correct structure, the best results are given by the permutation symbolic coupling traces (figure 3.2 (b)). 


\begin{tabular}{|c|c|c|c|c|c|c|c|c|c|c|}
\hline \multirow[b]{2}{*}{ couplings } & \multicolumn{2}{|c|}{$\begin{array}{c}\text { epoch } \mathbf{1} \\
t=1, \ldots, 199\end{array}$} & \multicolumn{2}{|c|}{$\begin{array}{c}\text { epoch 2 } \\
t=200, \ldots, 299\end{array}$} & \multicolumn{2}{|c|}{$\begin{array}{c}\text { epoch } 3 \\
t=300, \ldots, 350\end{array}$} & \multicolumn{2}{|c|}{$\begin{array}{c}\text { epoch } 4 \\
t=351, \ldots, 699\end{array}$} & \multicolumn{2}{|c|}{$\begin{array}{c}\text { epoch } 5 \\
t=700, \ldots, 1000\end{array}$} \\
\hline & $\tau$ & $a_{i j}(\tau, t)$ & $\tau$ & $a_{i j}(\tau, t)$ & $\tau$ & $a_{i j}(\tau, t)$ & $\tau$ & $a_{i j}(\tau, t)$ & $\tau$ & $a_{i j}(\tau, t)$ \\
\hline$x_{1}$ & 1 & 0.3 & 1 & 0.3 & 1 & 0.3 & 1 & 0.3 & 1 & 0.3 \\
\hline$x_{2}$ & 1 & 0.3 & 1 & 0.3 & 1 & 0.3 & 1 & 0.3 & 1 & 0.3 \\
\hline$x_{3}$ & 1 & 0.4 & 1 & 0.4 & 1 & 0.4 & 1 & 0.4 & 1 & 0.4 \\
\hline$x_{4}$ & 1 & 0.3 & 1 & 0.3 & 1 & 0.3 & 1 & 0.3 & 1 & 0.3 \\
\hline$x_{5}$ & 1 & 0.3 & 1 & 0.3 & 1 & 0.3 & 1 & 0.3 & 1 & 0.3 \\
\hline$x_{1} \rightarrow x_{2}$ & 2 & -0.7 & 5 & -0.5 & 5 & -0.5 & 5 & -0.5 & 5 & -0.5 \\
\hline$x_{2} \rightarrow x_{1}$ & 1 & 0.7 & 1 & 0.7 & 1 & 0.7 & 1 & 0.7 & 3 & 0.6 \\
\hline$x_{3} \rightarrow x_{1}, x_{2}, x_{4}, x_{5}$ & 1 & 0.3 & 1 & 0.3 & 1 & 0.3 & 1 & 0.3 & 1 & 0.3 \\
\hline$x_{4} \rightarrow x_{5}$ & 3 & 0.7 & 3 & 0.7 & 2 & -0.7 & 3 & 0.7 & 3 & 0.7 \\
\hline
\end{tabular}

Table 3.1: The table shows the time lags and coupling strength parameters for a time-dependent ARmodel with five variables, displaying univariate and feedback coupling, as well as a common driver. The model equation for $x_{1}$ during the fifth epoch is e.g. given by $x_{1}(t)=0.3 x_{1}(t-$ $1)+0.6 x_{2}(t-3)+0.3 x_{3}(t-1)+\epsilon_{1}(t)$.

Since the coupling from $x_{3}$ to all the other variables in the model is comparatively weak, it is only correctly identified by Granger causality and the ESCT. Cross-correlation and mutual information show some additional spurious couplings while the PESCT finds no couplings at all. The common driver effects (identified couplings between variables that are uncoupled) are only shown by the cross-correlation. Omitting the $x_{3}$ from the pool of available data to compute the coupling structure has no effect on all measures, since ESCT, PESCT, EXCORR, and EMI only work on a bivariate basis, and Granger causality is corrected via the partial Granger causality extension.

The full resulting coupling structure for all measures is given in figure 3.3 (b) - (f). 


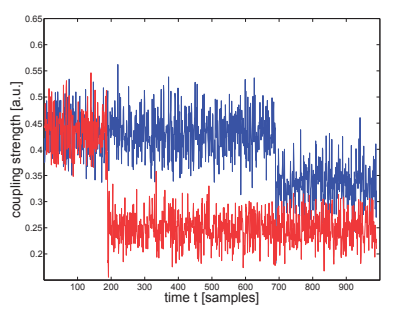

(a) ECGC

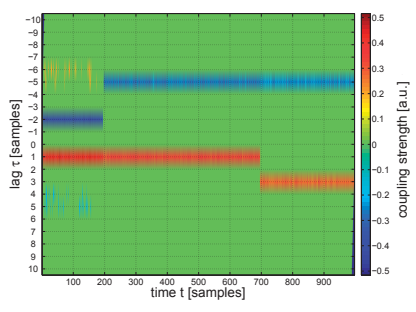

(b) ESCT

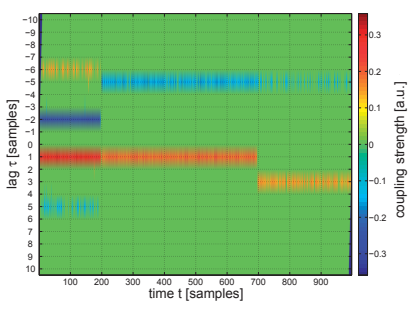

(c) PESCT

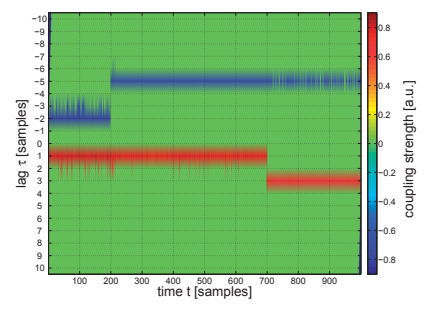

(d) EXCORR

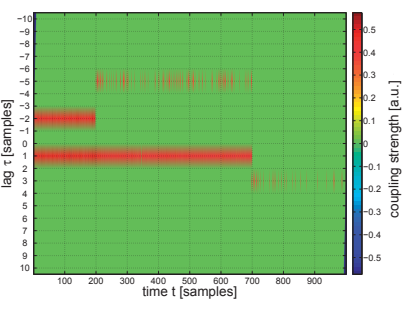

(e) EMI

Figure 3.1: This figure shows the significant results of the regarded methods for the coupling structure of the AR-model given in table 3.1. In (a) the red colour depicts the coupling from $x_{1}$ to $x_{2}$, for blue it is vice versa. In (b) - (e) red stands for symmetric coupling and blue for diametric. 


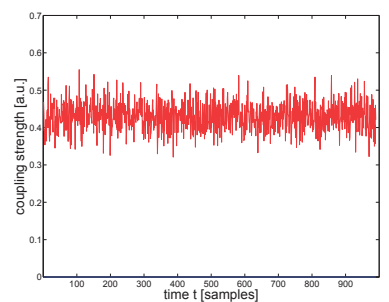

(a) ECGC

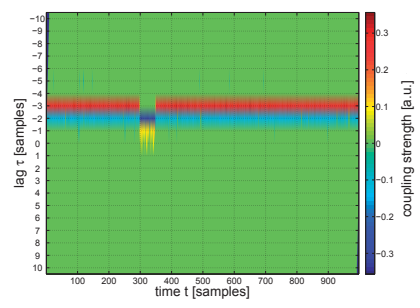

(b) ESCT

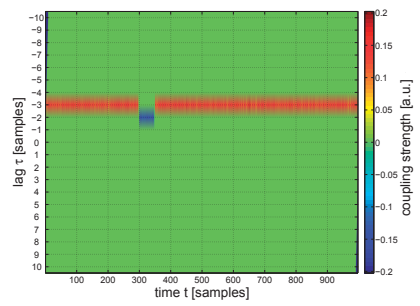

(c) PESCT

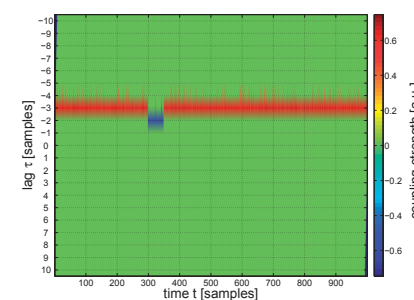

(d) EXCORR

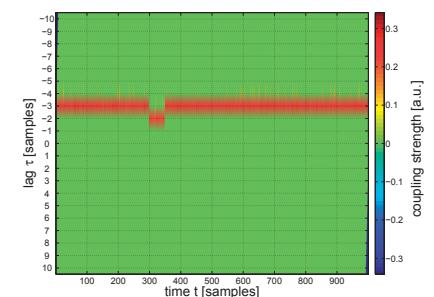

(e) EMI

Figure 3.2: This figure shows the significant results of the regarded methods for the coupling structure of the AR-model given in table 3.1. In (a) the red colour depicts the coupling from $x_{4}$ to $x_{5}$. In (b) - (e) red stands for symmetric coupling and blue for diametric.

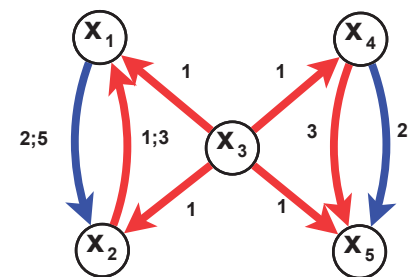

(a) original

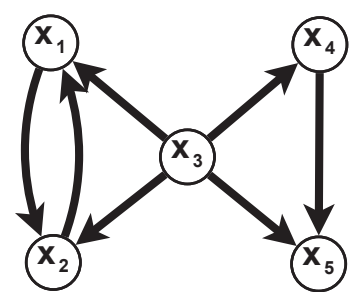

(b) ECGC

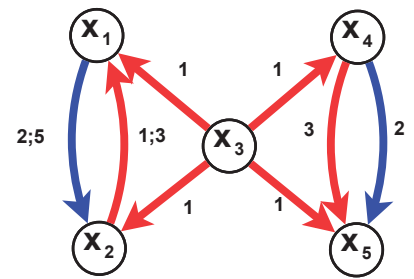

(c) $\mathrm{ESCT}$

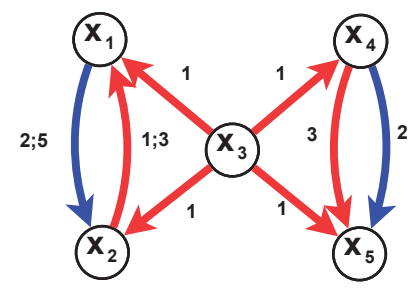

(d) PESCT

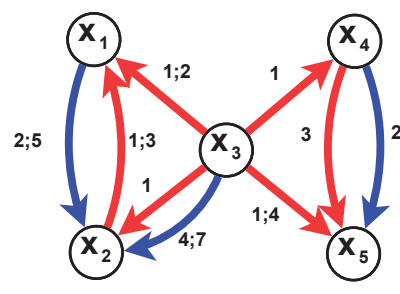

(e) EXCORR

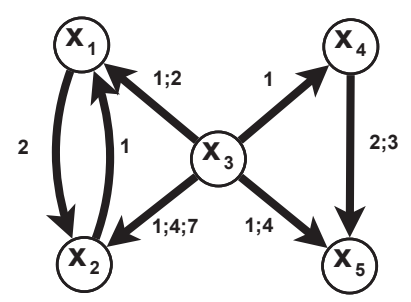

(f) EMI

Figure 3.3: This figure shows the original (a) and the resulting ((b) - (f)) coupling structures from the regarded methods for the AR-model given in table 3.1. The colours of the arrows depict symmetric (red) and diametric (blue) coupling. For results from methods not able to distinguish between these, there are black arrows. The numbers show the occurring time lags. 


\section{AR-model with multiple lag coupling}

The second model is a bivariate AR-process incorporating univariate coupling over multiple time lags for one variable. The coupling structure is given in figure 3.5 and the model parameters in table 3.2. The standard deviation of the white noise innovative term $\epsilon$ is $\sigma=0.01$, the length of the regarded time series is again $N=1000$ and $M=1000$ realisations have been used. The significance level has been set to $\alpha=0.05$ and the model order has been determined as $\Omega=10$ via the BIC-method while the AIC-result recommended $\Omega=1$.

\begin{tabular}{ccccccc} 
& \multicolumn{2}{c}{ epoch 1 } & \multicolumn{2}{c}{ epoch 2 } & \multicolumn{2}{c}{ epoch 3 } \\
& \multicolumn{2}{c}{$t=1, \ldots, 199$} & \multicolumn{2}{c}{$t=200, \ldots, 699$} & \multicolumn{2}{c}{$t=700, \ldots, 1000$} \\
couplings & $\tau$ & $a_{i j}(\tau, t)$ & $\tau$ & $a_{i j}(\tau, t)$ & $\tau$ & $a_{i j}(\tau, t)$ \\
\hline$x_{1}$ & $1 ; 2 ; 3$ & $0.5 ;-0.3 ; 0.2$ & $1 ; 2 ; 3$ & $0.5 ;-0.3 ; 0.2$ & $1 ; 2 ; 3$ & $0.5 ;-0.3 ; 0.2$ \\
$x_{2}$ & $1 ; 2 ; 3$ & $0.2 ; 0.5 ;-0.4$ & $2 ; 3 ; 5$ & $0.5 ;-0.4 ; 0.2$ & $2 ; 3 ; 5$ & $0.5 ;-0.4 ; 0.2$ \\
\hline$x_{2} \rightarrow x_{1}$ & $4 ; 7$ & $0.7 ;-0.8$ & $4 ; 7$ & $0.7 ;-0.8$ & $2 ; 7$ & $0.7 ;-0.8$
\end{tabular}

Table 3.2: The table shows the time lags and coupling strength parameters for a time-dependent ARmodel with two variables, displaying univariate coupling across multiple time lags. The model equation for $x_{1}$ during the first epoch is e.g. given by $x_{1}(t)=0.5 x_{1}(t-1)-0.3 x_{1}(t-2)+$ $0.2 x_{1}(t-3)+0.7 x_{2}(t-4)-0.8 x_{2}(t-7)+\epsilon_{1}(t)$.

Again, all measures except the ensemble mutual information (figure 3.4 (e)) identify the correct coupling structure during the different epochs. The resulting overall coupling structure for the second example is shown in figure 3.5 (b) - (f). 


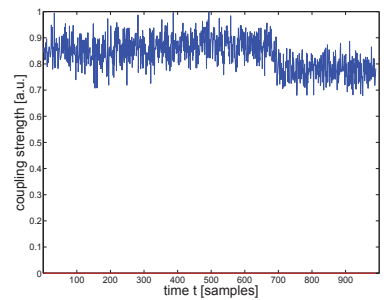

(a) ECGC

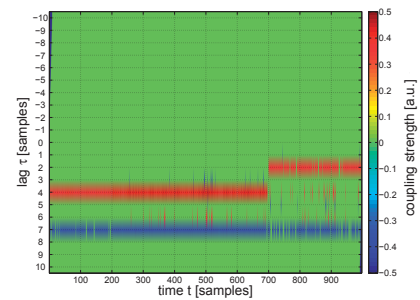

(b) ESCT

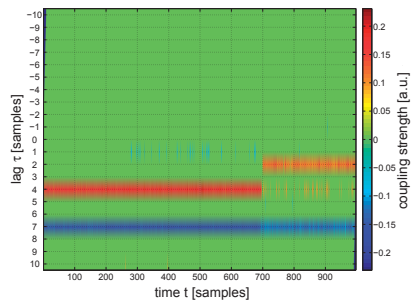

(c) PESCT

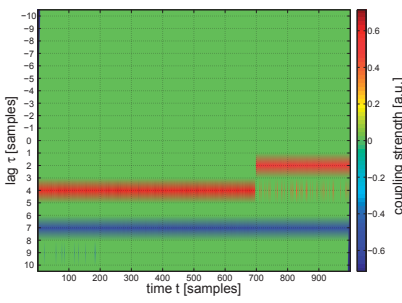

(d) EXCORR

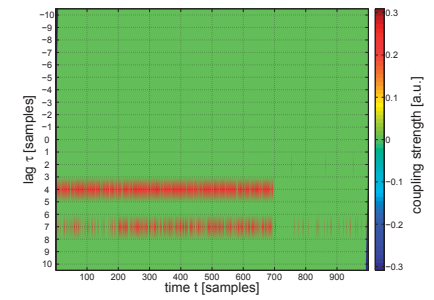

(e) EMI

Figure 3.4: This figure shows the significant results of the regarded methods for the coupling structure of the AR-model given in table 3.2. In (a) the blue colour depicts the coupling from $x_{2}$ to $x_{1}$. In (b) - (e) red represents symmetric coupling and blue diametric coupling.

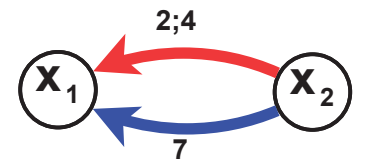

(a) original

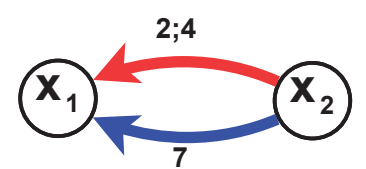

(d) PESCT
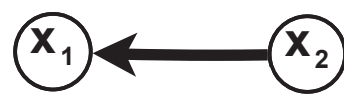

(b) ECGC

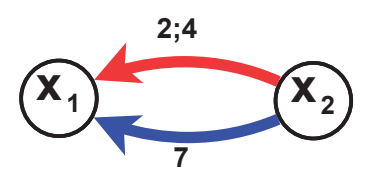

(e) EXCORR

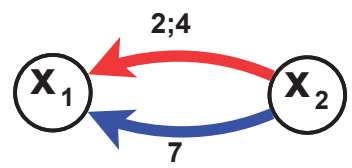

(c) ESCT

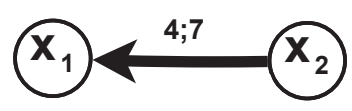

(f) EMI

Figure 3.5: This figure shows the original (a) and the resulting ((b) - (f)) coupling structures from the regarded methods for the AR-model given in table 3.2. The colours of the arrows depict symmetric (red) and diametric (blue) coupling. For results from methods not able to distinguish between these, there are black arrows. The numbers show the occurring time lags. 


\subsubsection{Nonlinear models}

Typically, most natural processes exhibit nonlinear behaviour like saturation, hysteresis, synchronisation, different regimes, or chaotic behaviour. Take e.g. the climate system or the baroreflex in the cardiovascular system. In order to check whether the regarded coupling measures also work for nonlinear systems three different examples are considered.

\section{Linearly coupled logistic maps}

The first model is the logistic map, originally derived to describe population dynamics [Ver38]. Consider the fraction $x(t)$ of the current population $y(t)$ and the maximum population $y_{\max }$. The time development can, in a simple case, be modeled by taking the reproduction and the death rate as proportional to the current values of $x(t)$ and $1-x(t)$ with proportionality factors $a_{\text {reprod }}$ and $a_{\text {death }}$, respectively. With $r=a_{\text {reprod }} a_{\text {death }}$ one obtains

$$
x(t)=r \cdot x(t-1) \cdot(1-x(t-1)) .
$$

This model exhibits different dynamic behaviour dependent on the choice of the parameter $r$. Here we regard the chaotic case for $r=4$.

A system of logistic maps with time-dependent coupling is described by the following equation.

$$
x_{j}(t)=a_{j j}\left(\tau_{j j}, t\right) \cdot r \cdot x_{j}\left(t-\tau_{j j}\right) \cdot\left(1-x_{j}\left(t-\tau_{j j}\right)\right)+\sum_{i \neq j}^{n_{v a r}} a_{i j}\left(\tau_{i j}, t\right) \cdot x_{i}\left(t-\tau_{i j}\right), \quad j \in\left\{1, \ldots, n_{v a r}\right\}
$$

The parameters for the case with linear coupling are given in table 3.3 and the coupling structure is also represented in figure 3.9 (a). As before, the data used for the analysis consists of $N=1000$

data points taken after a transient phase of 9000 data points and $M=1000$ realisations. Significance is set to $\alpha=0.05$ and a model order of $\Omega=12$ for the Granger causality has been used according to the BIC (AIC suggested $\Omega=1$ ). Figure 3.6 shows the resulting significant coupling structure between the variables $x_{1}$ and $x_{2}$. Although there is no coupling between these two variables in the second epoch, all measures, except Granger causality (figure 3.6 (e)), show the spurious coupling stemming from the coupling of the third variable $x_{3}$ to $x_{1}$ and $x_{2}$. However, in this case the coupling at $\operatorname{lag} \tau=0$ indicates a common driver. The lag $\tau=1$ coupling from $x_{1}$ to $x_{2}$ is not detected by any of the methods, except Granger causality. The coupling structure for $x_{1}$ and $x_{3}$ is shown in figure 3.7. The lag $\tau=5$ driving from $x_{3}$ to $x_{1}$ in the second and third epoch is correctly identified by all methods. However, all methods, except the mutual information (figure 3.7 (e)), show a spurious coupling at $\tau=8$. Even the partial Granger 


\begin{tabular}{ccccccc} 
& \multicolumn{2}{c}{ epoch 1 } & \multicolumn{2}{c}{ epoch 2 } & \multicolumn{2}{c}{ epoch 3 } \\
& $t=1, \ldots, 199$ & \multicolumn{2}{c}{$t=200, \ldots, 699$} & \multicolumn{2}{c}{$t=700, \ldots, 1000$} \\
couplings & $\tau_{i j}$ & $a_{i j}\left(\tau_{i j}, t\right)$ & $\tau_{i j}$ & $a_{i j}\left(\tau_{i j}, t\right)$ & $\tau_{i j}$ & $a_{i j}\left(\tau_{i j}, t\right)$ \\
\hline$x_{1}$ & 1 & 0.5 & 1 & 0.6 & 1 & 0.6 \\
$x_{2}$ & 1 & 0.6 & 1 & 0.6 & 1 & 0.4 \\
$x_{3}$ & 1 & 1 & 1 & 1 & 1 & 1 \\
\hline$x_{1} \rightarrow x_{2}$ & 0 & 0 & 0 & 0 & 1 & 0.3 \\
$x_{2} \rightarrow x_{1}$ & 3 & 0.5 & 0 & 0 & 0 & 0 \\
$x_{3} \rightarrow x_{1}$ & 0 & 0 & 5 & 0.4 & 1 & 0.3 \\
$x_{3} \rightarrow x_{2}$ & 5 & 0.4 & 1 & 0.4 & 5 & 0.3
\end{tabular}

Table 3.3: The table shows the time lags and coupling strength parameters for three logistic maps with time-dependent coupling. The model equation for $x_{1}$ during the first epoch is e.g. given by $x_{1}(t)=0.5 \cdot 4 \cdot x_{1}(t-1) \cdot\left(1-x_{1}(t-1)\right)+0.5 \cdot x_{2}(t-3)$.

(figure 3.7 (a)) causality shows a weak coupling there. This, of course, stems from the strong coupling between $x_{2}$ and $x_{3}(\tau=5)$ and between $x_{1}$ and $x_{2}(\tau=3)$. The connections between $x_{2}$ and $x_{3}$ are depicted in figure 3.8. The correct structure is comparatively well identified by all measures. The symbolic coupling traces and the permutation symbolic coupling traces even correctly show the decrease in coupling strength in the third epoch. Granger causality, however, here shows an increase in the coupling strength. In this case, the increase originates in the switched on coupling from $x_{1}$ to $x_{2}$, while $x_{1}$ is still strongly driven by $x_{3}$. The resulting overall coupling structure for all measures is shown in figure 3.9 (b) - (f). 


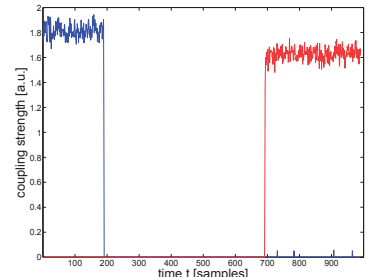

(a) ECGC

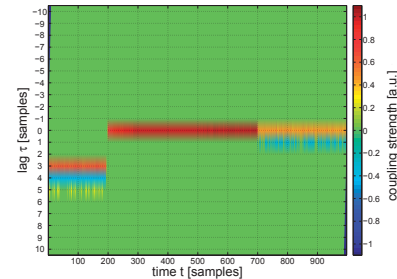

(b) ESCT

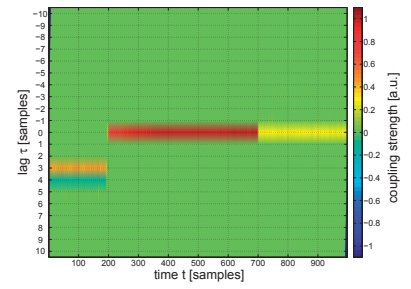

(c) PESCT

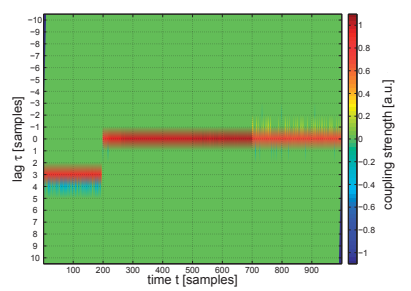

(d) EXCORR

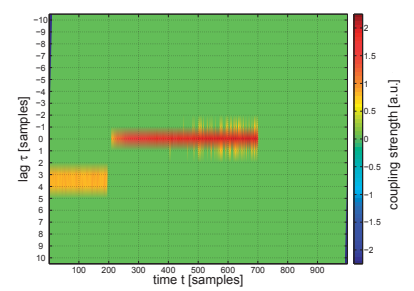

(e) EMI

Figure 3.6: This figure shows the significant results of the regarded methods for the coupling structure of the coupled logistic maps given in table 3.3. In (a) the blue colour depicts the coupling from $x_{2}$ to $x_{1}$, for the red colour it is vice versa. In (b) - (e) red represents symmetric coupling and blue diametric coupling.

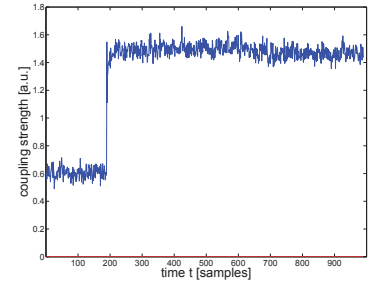

(a) ECGC

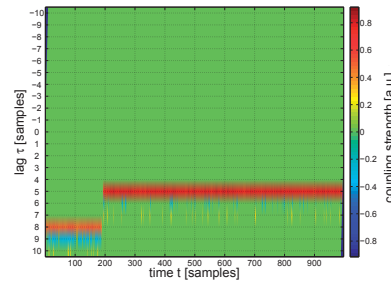

(b) ESCT

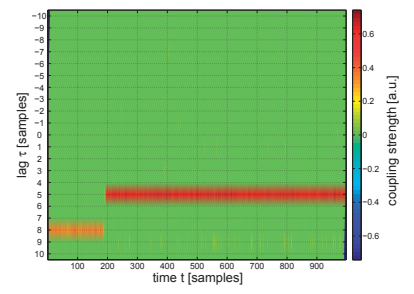

(c) PESCT

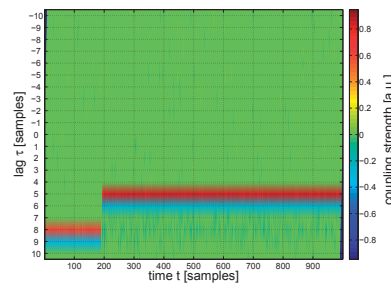

(d) EXCORR

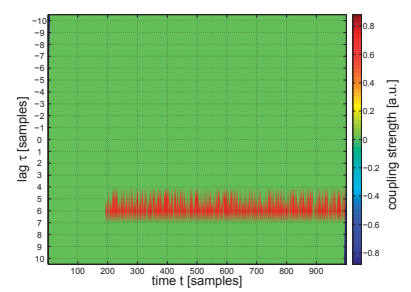

(e) EMI

Figure 3.7: This figure shows the significant results of the regarded methods for the coupling structure of the coupled logistic maps given in table 3.3. In (a) the blue colour depicts the coupling from $x_{3}$ to $x_{1}$. In (b) - (e) red represents symmetric coupling and blue diametric coupling. 


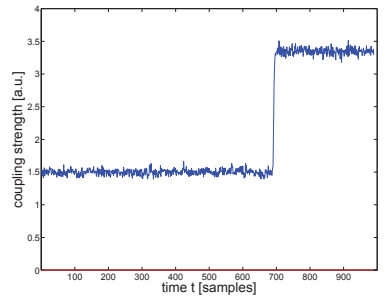

(a) ECGC

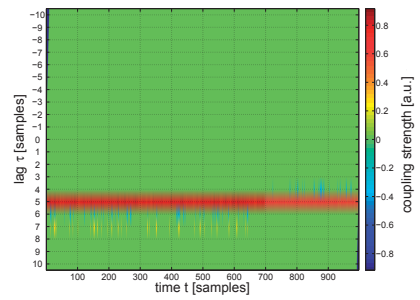

(b) ESCT

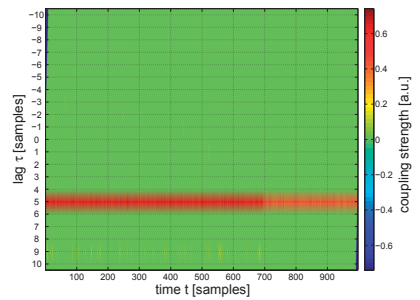

(c) PESCT

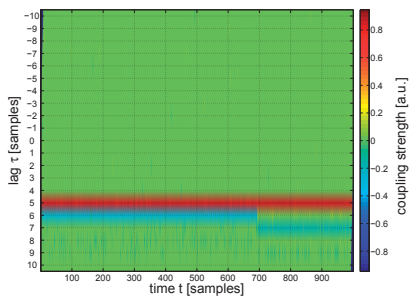

(d) EXCORR

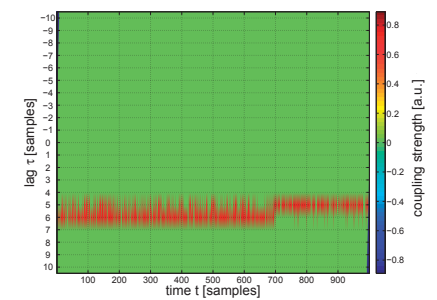

(e) EMI

Figure 3.8: This figure shows the significant results of the regarded methods for the coupling structure of the coupled logistic maps given in table 3.3. In (a) the blue colour depicts the coupling from $x_{3}$ to $x_{2}$. In (b) - (e) red represents symmetric coupling and blue diametric coupling.

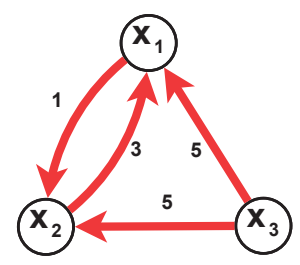

(a) original

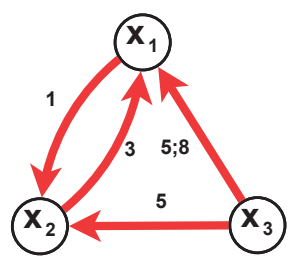

(d) PESCT

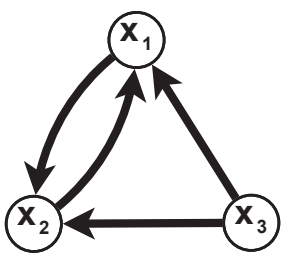

(b) ECGC

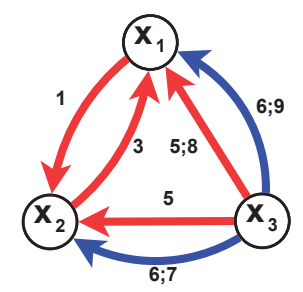

(e) EXCORR

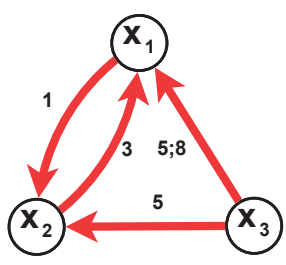

(c) ESCT

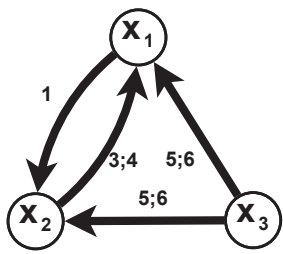

(f) EMI

Figure 3.9: This figure shows the original (a) and the resulting ((b) - (f)) coupling structures from the regarded methods for the linearly coupled logistic maps given in table 3.3. The colours of the arrows depict symmetric (red) and diametric (blue) coupling. For results from methods not able to distinguish between these, there are black arrows. The numbers show the occurring time lags. 


\section{Nonlinearly coupled logistic maps}

The second model in this subsection consists again of logistic maps. This time the coupling occurs via a quadratic term in the driving variable. The full parameters for the model are given in table 3.4 while the coupling structure is also depicted in figure 3.11 (a). Again $N=1000$ data points are considered for $M=1000$ realisations across to different coupling epochs. The model order for the Granger causality has been chosen as $\Omega=10$ as a compromise between $\Omega=8$ (AIC) and $\Omega=23$ (BIC) and the significance level has been set to $\alpha=0.1$. The higher value of $\alpha$ here was chosen due to the attenuated sensitivity of the regarded measures for nonlinear effects. At $\alpha=0.05$ the weaker coupling in the second epoch could not be detected by any of the methods except the ECGC. All regarded methods were able to correctly identify the coupling

\begin{tabular}{ccccc} 
& \multicolumn{2}{c}{ epoch 1 } & \multicolumn{2}{c}{ epoch 2 } \\
& \multicolumn{1}{c}{$t=1, \ldots, 499$} & \multicolumn{2}{c}{$t=500, \ldots, 1000$} \\
couplings & $\tau_{i j}$ & $a_{i j}\left(\tau_{i j}, t\right)$ & $\tau_{i j}$ & $a_{i j}\left(\tau_{i j}, t\right)$ \\
\hline$x_{1}$ & 1 & 1 & 1 & 1 \\
$x_{2}$ & 1 & 0.5 & 1 & 0.7 \\
\hline$x_{1, \tau_{1}} \cdot x_{1, \tau_{2}} \rightarrow x_{2}$ & $1 ; 5$ & 0.5 & $1 ; 5$ & 0.3
\end{tabular}

Table 3.4: The table shows the time lags and coupling strength parameters for two logistic maps with time-dependent coupling. The nonlinear coupling term depends on two different time lags, denoted here as $\tau_{1}$ and $\tau_{2}$. The model equation for $x_{2}$ during the first epoch is e.g. given by $x_{2}(t)=0.5 \cdot 4 \cdot x_{2}(t-1) \cdot\left(1-x_{2}(t-1)\right)+0.5 \cdot x_{1}(t-1) \cdot x_{1}(t-5)$.

structure (see figure 3.10), although the EXCORR- (figure 3.10 (d)) and the EMI-measure (figure 3.10 (e)) show results smeared over several lags and EMI is not able to recognise the coupling in the second epoch for the chosen significance level. The other three measures (figure 3.10 (a) - (c)) even reflect the correct relation of the coupling strengths for the two epochs. The results for all methods are summarised in figure 3.11 (b) - (f). 


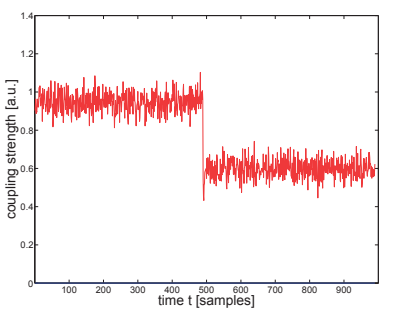

(a) ECGC

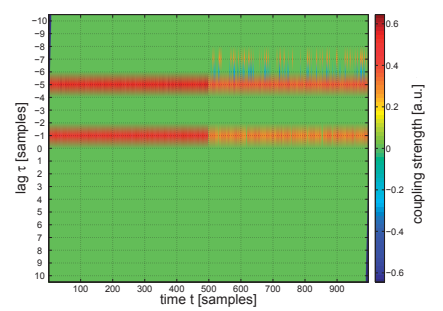

(b) ESCT

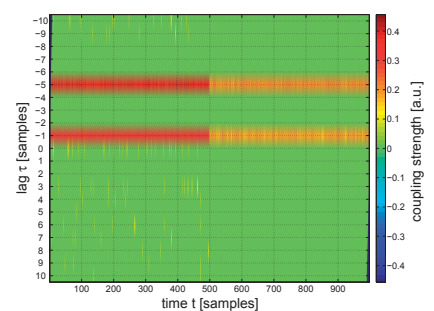

(c) PESCT

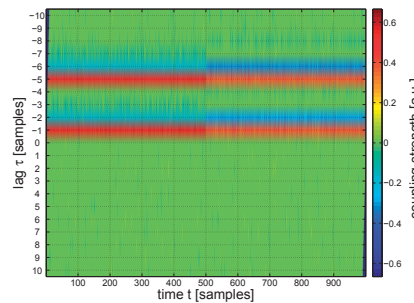

(d) EXCORR

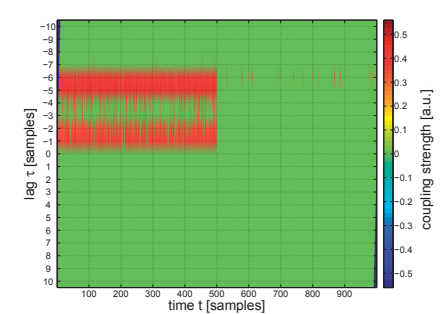

(e) EMI

Figure 3.10: This figure shows the significant results of the regarded methods for the coupling structure of the coupled logistic maps given in table 3.4. In (a) the red colour depicts the coupling from $x_{1}$ to $x_{2}$. In (b) - (e) red represents symmetric coupling and blue diametric coupling.

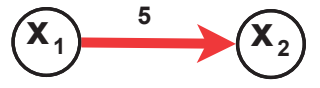

(a) original

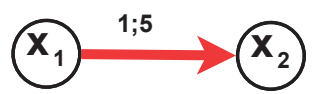

(d) PESCT

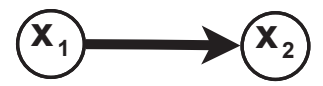

(b) ECGC

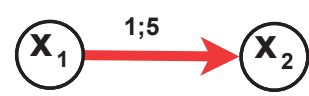

(e) EXCORR

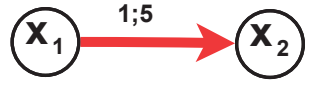

(c) $\mathrm{ESCT}$

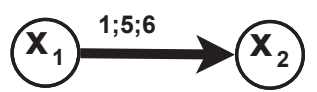

(f) EMI

Figure 3.11: This figure shows the original (a) and the resulting ((b) - (f)) coupling structures from the regarded methods for the nonlinearly coupled logistic maps given in table 3.4. The colours of the arrows depict symmetric (red) and diametric (blue) coupling. For results from methods not able to distinguish between these, there are black arrows. The numbers show the occurring time lags. 


\section{NAARX-model}

Nonlinear autoregressive additive models are used in a wide field of applications like medicine $\left[\mathrm{WMB}^{+}\right.$06], climate research [DBR00], and in theoretical [CT93, VK97] as well as practical [VK99, VKAK99, VSKM99] nonlinear optics. This model class represents a generalisation of AR-models, since the way future values of a process $X$ depend on past values can now be modeled by an arbitrary function $f$, while the additivity still allows for a separate analysis of different time delays. Nonlinear effects in AR-models are masked by employing preceding values. Thus it is difficult to interpret the resulting parameters as effect size because these rather represent the fitting process. Thus, NAAR-models are easier to interpret than multidimensional models. The time-dependent version of this class of models can be described in the following way,

$$
x(t)=\sum_{\tau=1}^{\omega} f(x(t-\tau), \tau, t)+\epsilon(t) .
$$

In the same way that AR-models can be extended for the multivariate case, the time-dependent NAARX-model describes a nonlinear additive autoregressive model with external inputs and is given by

$$
x_{j}(t)=\sum_{i=1}^{n_{\text {var }}} \sum_{\tau=1}^{\omega} f_{i j}\left(x_{i}(t-\tau), \tau, t\right)+\epsilon_{j}(t), \quad j \in\left\{1, \ldots, n_{\text {var }}\right\} .
$$

Equations (3.1) and (3.2) show the actual model used for testing the coupling analysis methods.

$$
\begin{aligned}
& x_{1}(t)=\left\{\begin{array}{lc}
a_{11}(\tau, t) x_{1}(t-\tau)+\delta(t)+\epsilon_{1}(t), & \left|x_{2}(t-\tau)\right| \leq 0.25 \\
a_{11}(\tau, t) x_{1}(t-\tau)-\delta(t)+\epsilon_{1}(t), & \text { otherwise }
\end{array}\right. \\
& x_{2}(t)=0.25 \cdot \epsilon_{2}(t)
\end{aligned}
$$

So, the second variable, or in this case, the external input is given by an independent Gaussian process with an expected value of zero and a variance of 0.25 . The remaining parameters of the model are shown in table 3.5 and the coupling structure is represented in figure 3.13 (a).

The model order for the Granger causality analysis has been chosen as $\Omega=13$ according to the BIC. The AIC would have suggested $\Omega=2$. The significance threshold is set to $\alpha=0.05$. Without further preprocessing only the mutual information is able to show the correct coupling 


\begin{tabular}{ccccccc} 
& \multicolumn{3}{c}{ epoch 1 } & \multicolumn{3}{c}{ epoch 2 } \\
& \multicolumn{3}{c}{$t=1, \ldots, 499$} & \multicolumn{3}{c}{$t=500, \ldots, 1000$} \\
couplings & $\tau$ & $a_{i j}(\tau, t)$ & $\delta$ & $\tau$ & $a_{i j}(\tau, t)$ & $\delta$ \\
\hline$x_{1}$ & 1 & 0.2 & - & 1 & 0.2 & - \\
\hline$x_{2} \rightarrow x_{1}$ & 1 & - & 0.05 & 3 & - & -0.1
\end{tabular}

Table 3.5: The table shows the time lags and coupling parameters for a NAARX-model with timedependent coupling.

structure (see figure $3.12(\mathrm{e})$ ). When adding the quadratic term in the Granger causality formalism as described in subsection 2.3.4, Granger causality also finds the right coupling direction (figure 3.12 (a)). Using the simple extension described in section 2.4, also the symbolic coupling traces and the permutation symbolic coupling traces reveal correct structures (figure 3.12 (b) and (c)). Instead of using the original time series as input, the entries of the second variable have been substituted with their absolute values. This of course increases, depending on the number of variables regarded, the amount of time and effort needed to identify couplings, but is still simple and has low computational costs. The cross-correlation shows no couplings at all at the chosen significance level.

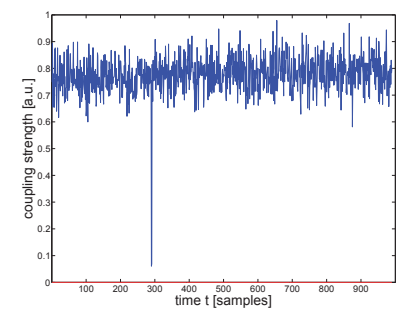

(a) ECGC

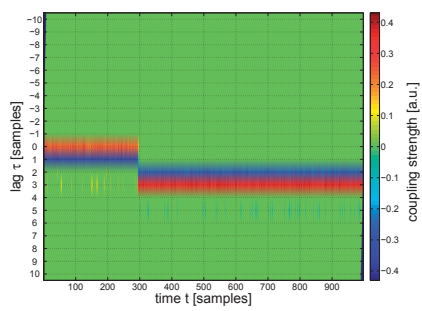

(b) $\mathrm{ESCT}$

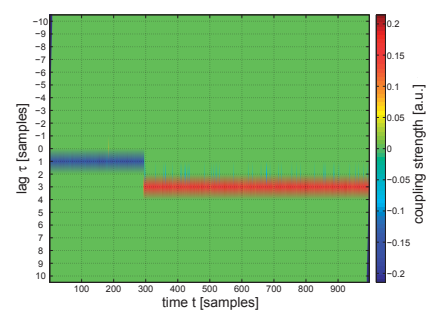

(c) PESCT

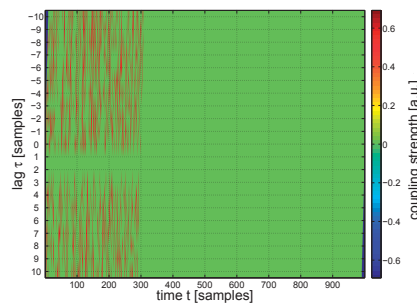

(d) EXCORR

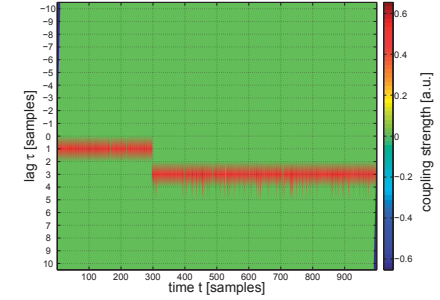

(e) EMI

Figure 3.12: This figure shows the significant results of the regarded methods for the coupling structure of the NAARX-model given in table 3.3. In (a) the blue colour depicts the coupling from $x_{2}$ to $x_{1}$. In (b) - (e) red represents symmetric coupling and blue diametric coupling. 
The resulting coupling structure for all measures, after their individual preprocessings, is represented in figure 3.13 (b) - (f).

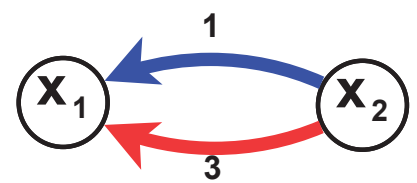

(a) original

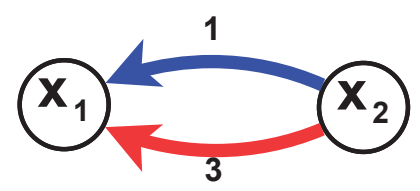

(d) PESCT

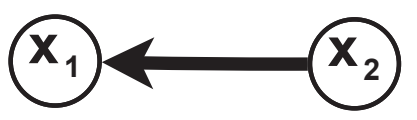

(b) ECGC

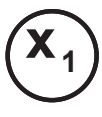

(e) EXCORR

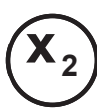

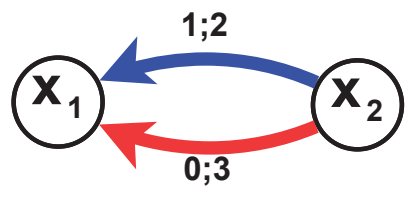

(c) ESCT

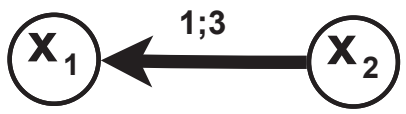

(f) EMI

Figure 3.13: This figure shows the original (a) and the resulting ((b) - (f)) coupling structures from the regarded methods for the NAARX-model given in table 3.5. The colours of the arrows depict symmetric (red) and diametric (blue) coupling. For results from methods not able to distinguish between these, there are black arrows. The numbers show the occurring time lags.

\subsubsection{Influence of ensemble size, coupling strength, and noise}

To assess the robustness of the different coupling analysis tools, three different tests are performed on an AR-model with two variables. The model parameters are given in table 3.6.

All measures have been able to identify the correct coupling structure of this model (see subsection 3.1.1). To assess quality of the results for Granger causality are compared with a reference result (Granger causality for the system with no added noise and an ensemble size of $M=1000)$. The quality parameter is then computed as the percentage of matching results for each time point. For all other methods the results are checked against a reference matrix containing the actual couplings at each time point and lag. The quality parameter is the cross-correlation between the results and the reference matrix. So, in each case, a value of one represents the best possible outcome. The results of each method have been subject to a significance test with a significance level of $\alpha=0.05$. The first test deals with dependence

of the results on the size of the ensemble. It has been changed starting from $M=10$ up to $M=1000$ and the results are shown in figure 3.14. For Granger causality (figure 3.14 (a)) no meaningful results are obtained for an ensemble size smaller than $M=30$ because of the model order $\Omega=10$. 


\begin{tabular}{ccccccc} 
& \multicolumn{2}{c}{ epoch 1 } & \multicolumn{2}{c}{ epoch 2 } & \multicolumn{2}{c}{ epoch 3 } \\
& $t=1, \ldots, 199$ & \multicolumn{2}{c}{$t=200, \ldots, 699$} & \multicolumn{2}{c}{$t=700, \ldots, 1000$} \\
couplings & $\tau$ & $a_{i j}(\tau, t)$ & $\tau$ & $a_{i j}(\tau, t)$ & $\tau$ & $a_{i j}(\tau, t)$ \\
\hline$x_{1}$ & 1 & 0.3 & 1 & 0.3 & 1 & 0.3 \\
$x_{2}$ & 1 & 0.3 & 1 & 0.3 & 1 & 0.3 \\
\hline$x_{1} \rightarrow x_{2}$ & 2 & -0.7 & 5 & -0.7 & 5 & -0.7 \\
$x_{2} \rightarrow x_{1}$ & 1 & 0.7 & 1 & 0.7 & 3 & 0.7
\end{tabular}

Table 3.6: The table shows the time lags and coupling strength parameters for a time-dependent ARmodel with two variables, for testing the robustness of the regarded coupling methods. The model equation for $x_{1}$ during the first epoch is e.g. given by $x_{1}(t)=0.3 x_{1}(t-1)+0.7 x_{2}(t-$ 1) $\epsilon_{1}(t)$.

In the second test the influence of the coupling strength is checked. Here, coupling strength is defined as the ratio of parameters describing dependence of one variable on the second variable and on the past of the first variable itself (e.g. $\frac{a_{21}}{a_{11}}$ ). The results for this case are shown in figure 3.15 .

To evaluate the methods' performance under the influence of noise, additive noise with increasing amplitudes is added to the time series. The signal-to-noise-ratio is computed as the mean amplitude of the signal before adding the noise divided by the variance of the noise. The results are shown in figure 3.16 . 


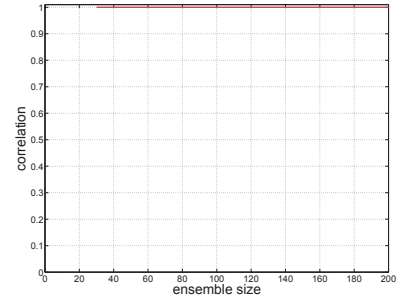

(a) ECGC

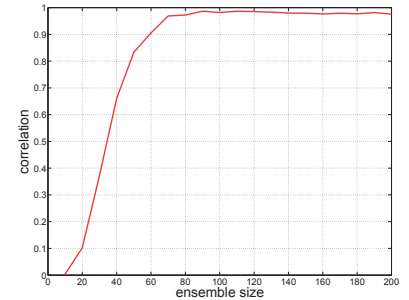

(b) ESCT

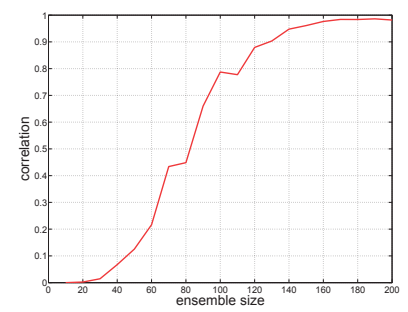

(c) PESCT

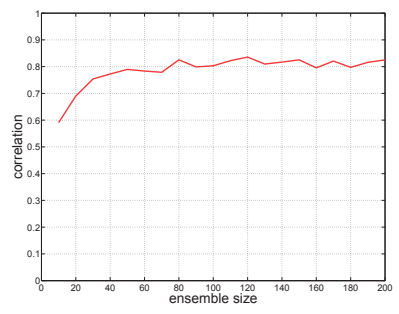

(d) EXCORR

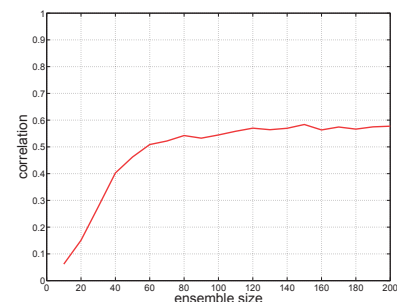

(e) EMI

Figure 3.14: This figure shows the quality of the results for each of the regarded coupling measures depending on the ensemble size.

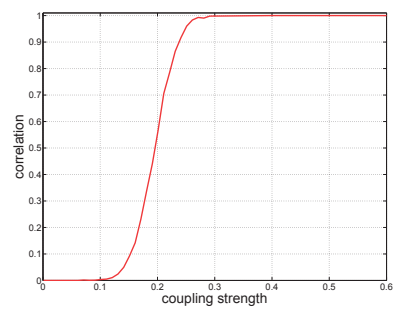

(a) ECGC

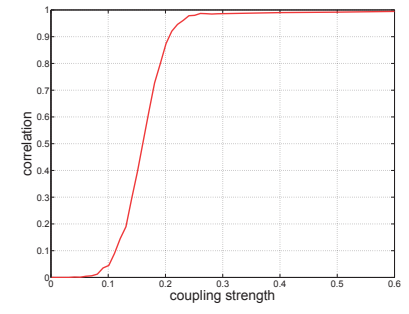

(b) ESCT

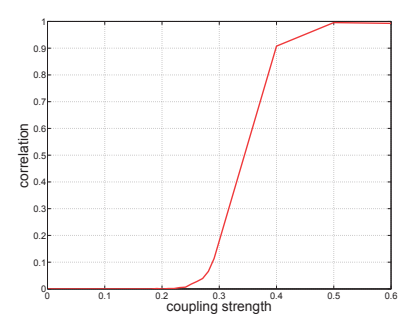

(c) PESCT

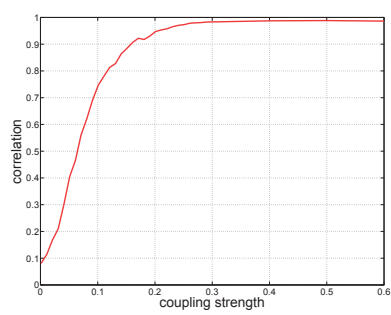

(d) EXCORR

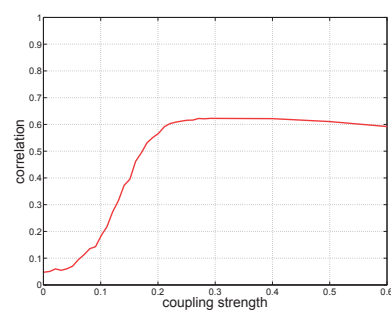

(e) EMI

Figure 3.15: This figure shows the quality of the results for each of the regarded coupling measures depending on the coupling strength. 


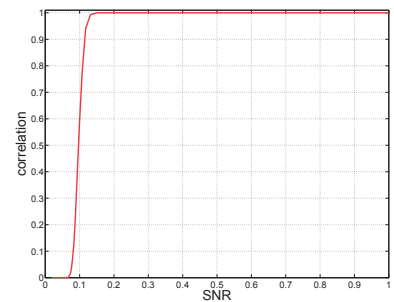

(a) ECGC

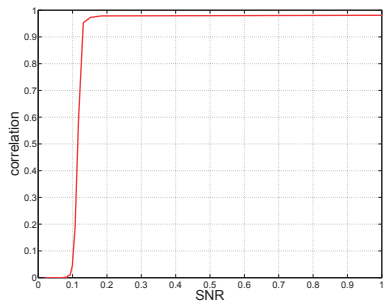

(b) ESCT

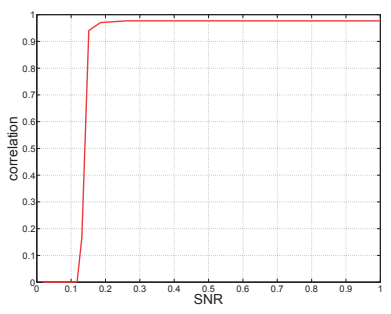

(c) PESCT

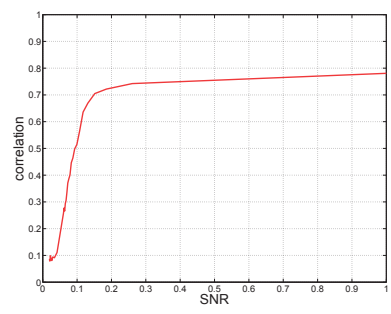

(d) EXCORR

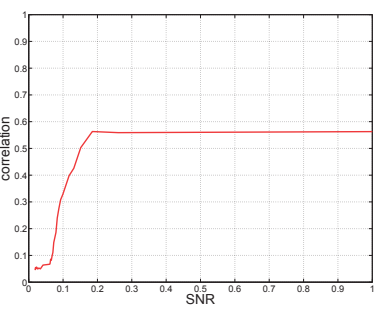

(e) EMI

Figure 3.16: This figure shows the quality of the results for each of the regarded coupling measures depending on the magnitude of additive noise.

\subsection{Discussion}

In the previous sections the methods of cross-correlation, mutual information, Granger causality, as well as two approaches of the symbolic coupling traces, all with an ensemble extension to allow for a time-resolved coupling analysis, have been tested on different theoretical models. The test on different AR-models showed all of the measures were able to reconstruct the original coupling structure in a mostly satisfying way. Only the ensemble mutual information showed some problems, e.g. for lower coupling strengths. Of course, this measure is the one requiring the biggest amount of data in order to adequately estimate the probability distributions. Another thing to keep in mind is the bivariate nature of the here regarded methods, except Granger causality, and thus one has to be careful with possible spurious couplings detected.

The second class of models, the measures were tested on, is characterised by its nonlinear nature. For systems with a simple additive coupling all methods again perform reasonably well. But here, even the partial Granger causality showed spurious coupling effects in coupling direction and coupling strength due to the nonlinear effects (compare figures 3.7 (a) and 3.8 (a), respectively). Again, the clearest results were delivered by Granger causality and the coupling traces. The coupling employed by the NAARX-model was only correctly identified by the mutual information. A simple extension to Granger causality and the symbolic methods 
enabled these to also find the correct couplings. So, depending on the behaviour of the system, the couplings in nonlinear systems might still display linear properties which can be detected by most coupling measures. Only when the coupling becomes purely nonlinear the methods fail without further extensions (except the mutual information). However, in most real-world applications at least partly linear couplings are very probable

The following tests depending on ensemble size, coupling strength, and noise were used to check the robustness of the measures' results. With an almost perfect reconstruction of the coupling direction for ensembles consisting of as little as 30 realisations, Granger causality gives the best performance here (figure 3.14 (a)). It is followed by the symbolic measures which reach a correlation of over 0.95 already below an ensemble size of $M=100$ (figures 3.14 (b) and $(\mathrm{c})$ ). Cross-correlation and mutual information also reach their peak performance relatively fast, but with much reduced quality (compare figures 3.14 (d) and (e)).

When regarding the dependence on coupling strength the best results in terms of sensitivity are obtained by the cross-correlation and the mutual information (figures 3.15 (d) and (e)). They are followed by the ensemble symbolic coupling traces and Granger causality (figures 3.15 (b) and (a)). If the coupling strength becomes to large, the two subsystems regarded essentially cannot be distinguished anymore and any coupling analysis is bound to fail. Here only the coupling direction identified by Granger causality can still be correctly derived for higher strength.

The addition of noise to the original signal was the final test performed. The best results here are obtained again by Granger causality and the two symbolic methods (figures 3.16 (a), (b), and (c)). Together with the mutual information (figure $3.16(\mathrm{e})$ ), whose performance in this case is worse than the other measures already at the beginning, deteriorating effects only occur for signal-to-noise-ratios below 0.2. Cross-correlation shows a constant reduction of quality with the rising noise level (figure $3.16(\mathrm{~d})$ ).

In summary, of the measures regarded here the best suited for an ensemble coupling analysis are the two symbolic measures and Granger causality. All three of them were able to identify the correct coupling directions and, in most cases, gave an adequate representation of the relative coupling strengths. Problems occur with nonlinear systems. But in some cases, these problems can be avoided by using e.g. a nonlinear model as the basis of Granger causality, or by using the absolute value of the regarded normalised time series. The first case, of course, may lead to larger amount of data needed to estimate the model parameters.

Due to its multivariate approach the ensemble partial Granger causality was the method best suited to exclude spurious couplings. But still the bivariate measures have a right to exist. One just has to be more careful when interpreting the results and look for example if certain lags could be explained by adding up lags from the other variables of the system. Of the two 
symbolic approaches, the permutation ensemble symbolic coupling traces are better qualified to avoid spurious couplings because of the finer coarse-graining. Of course the same also leads to a lower sensitivity when looking at lower coupling strengths.

In the end, which coupling measure to use, strongly depends on the system to analyse. However, the ensemble symbolic coupling traces are a good first choice requiring only small amounts of data, while giving information about the actual time lags present, about the relative coupling strengths, and about the effects of the influences (symmetric or diametric). 


\section{Application to data measured during an orthostatic test}

In this chapter applications to measured data from a real-world system and the corresponding results are presented, discussed and interpreted. The data stems from measurements of the cardiovascular system. In the cardiovascular system, transient behaviour occurs very often. These events may occur either due to the adaptive behaviour of the system throughout changes of states (e.g. orthostatic tests or change of sleep stages) or due to the recovery of the system after a severe disturbance (e.g., apnoea or arousals during sleep). These transient regime shifts contain important information about the underlying processes involved and the relations between state variables such as heart rate and blood pressure. In this section data from two studies will be analysed. The measured data encompasses an orthostatic test and thus belongs to the first group of adaptive behaviour. The data was taken from different subjects who performed the test once.

\subsection{Analysis}

The data set (see table 4.1) consists of 346 measurements on 195 female and 151 male subjects with ages ranging from 10 to 88 years $(45.5 \pm 16.1$ years for the women and $44.2 \pm 16.4$ years for the men) stemming from a study in $\left[\mathrm{BKO}^{+} 08\right]$. All subjects were measured in a supine position followed by an orthostatic test (in this case, simply getting up). After the test the measurement continued for a while in a standing position. The recorded variables comprise beat-to-beat-intervals, systolic and diastolic blood pressure, stroke volume (amount of blood pumped in one heart beat), and cardiac output (amount of blood pumped per minute; product of heart rate and stroke volume). The time of the orthostatic test was also marked in the files. For the analysis the ensembles have been built by aligning the measurements with the beginning of the orthostatic tests $(t=0)$ and regarding a hundred BBIs before and after the event, respectively. The averaged and normalised time series showing the typical behaviour (compare [NR07]) of the six regarded variables during the test are depicted in figure 4.1.

Due to the bigger amount of noise present in the measured data and the limited ensemble size, the significance level for the coupling analysis has been set to $\alpha=0.2$. For $\alpha=0.05$ no discernable structures have been present in the results. The model order has been chosen as $\Omega=10$ following the BIC (AIC suggested $\Omega=4$ ). Figure 4.2 exemplarily shows the results for the coupling analysis between BBI and SBP. 


$\begin{array}{cccc} & \text { younger }(\text { age } \leq 40) & \text { older }(\text { age }>40) & \text { total } \\ \text { female } & 84 & 111 & 195 \\ \text { male } & 74 & 77 & 151\end{array}$

Table 4.1: The table shows the number of available measurements with gender and age groups for the orthostatic test.

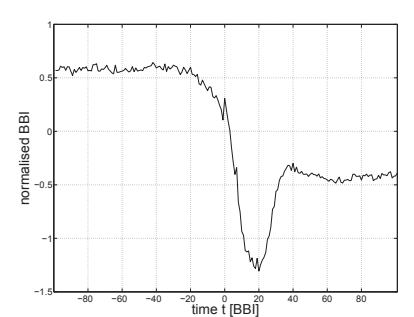

(a) BBI

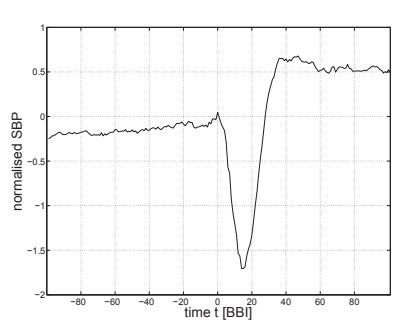

(b) SBP

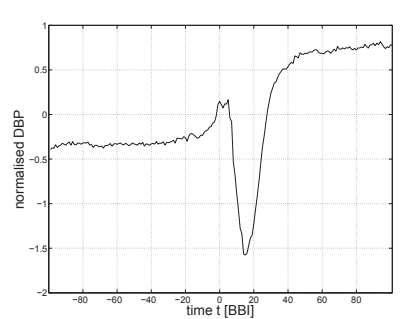

(c) DBP

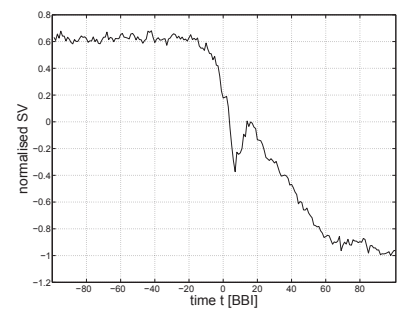

(d) SV

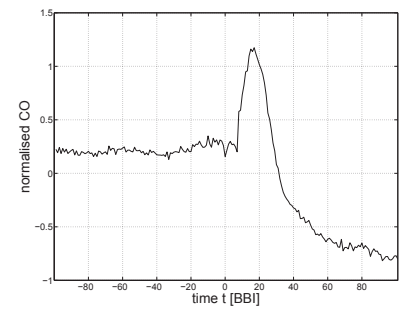

(e) $\mathrm{CO}$

Figure 4.1: This figure shows an example of the behaviour of the cardiovascular variables recorded in the study from $\left[\mathrm{BKO}^{+} 08\right]$ during an orthostatic test.

The results for all measures except the ESCT and the PESCT (see figure 4.2 (b) and (c)) are difficult to interpret. The stronger coarse graining, i.e the usage of fewer possible patterns, gives the ESCT an advantage in this case, because the coupling structure is easier discernible here than for the PESCT. Therefore, the ensemble symbolic coupling traces (see figure 4.2 (b)), showing the most promising results, will be used for the remaining analyses on this data set. The coupling structure for all variables according to the ESCT results is represented in figure 4.3.

The effect of the orthostatic test on the connection between all variables can be clearly seen as either a change in the relative coupling strength or the appearance of new significant lags. 


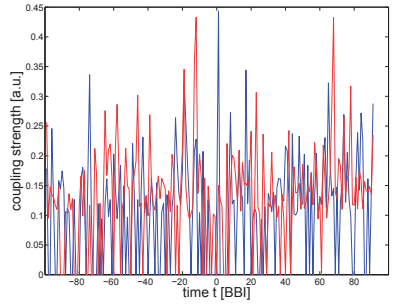

(a) ECGC

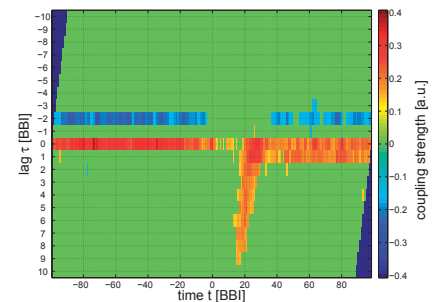

(b) $\mathrm{ESCT}$

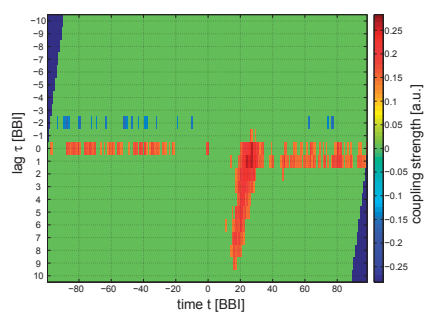

(c) PESCT

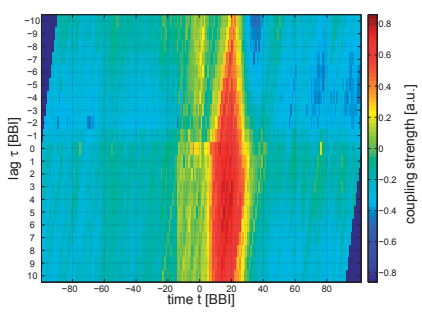

(d) EXCORR

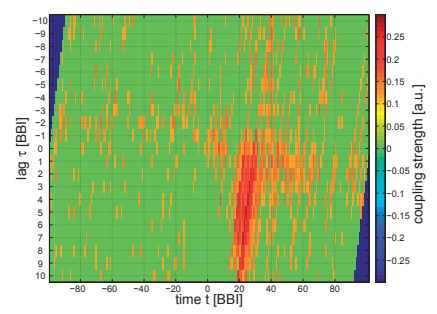

(e) EMI

Figure 4.2: This figure shows influences between BBI and SBP before, during, and after an orthostatic test.

In figure 4.3 (a) a diametric influence from the BBI to the SBP at $\tau=-2$ can be seen, as well as a symmetric behaviour at $\tau=0$. With the beginning of the event, the stationary structure is disrupted and several lags $(\tau>0)$ appear. Around 40 heartbeats after the event the original structure is mostly restored, showing only some lingering effects. Almost the same behaviour can be found in figure 4.3 (b), only here the structure is not restored even after the full 100 BBIs regarded. The same holds true for the connection between the BBI and the stroke volume (figure 4.3 (c)), although here the event causes just a disruption in the structure without new time lags appearing. In the coupling structure between BBIs and $\mathrm{CO}$ no discernible effect of the orthostatic test can be made out (figure 4.3 (c)). Figure 4.3 (e) shows an almost symmetric outburst of significant time lags around the event. Otherwise there is only a stationary coupling at $\tau=0$. The relationship between SBP and SV and CO is again mostly characterised by a break in the stationary structure (figures 4.3 (f) and $(\mathrm{g})$ ). In figure $4.3(\mathrm{~h})$ the ESCT show a reversal of the direction in the symmetric coupling between DBP and SV after the event $(\tau=-1$ to $\tau=1)$ via a short diametric coupling at $\tau=0$. The change of the coupling structure between DBP and CO, and between SV and CO is again mainly characterised by an increase of the relative coupling strength during the orthostatic test. Next, we want to look for changes in the coupling structure depending on gender and age. To retain a reasonable ensemble size the subjects are grouped according to an age below (younger) and above (older) 40 years. 


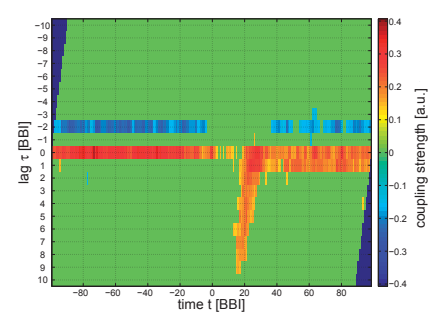

(a) BBI-SBP

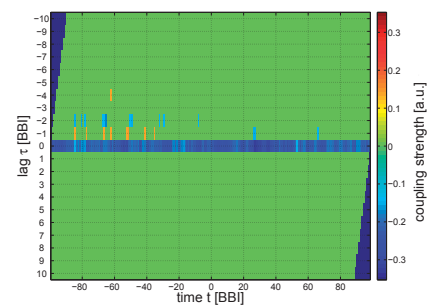

(d) BBI-CO

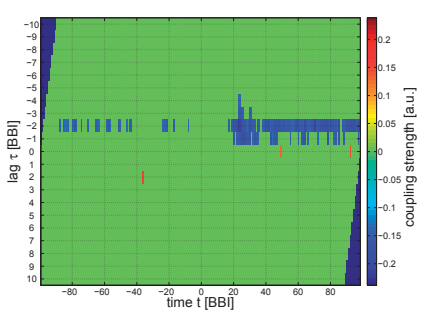

(g) SBP-CO

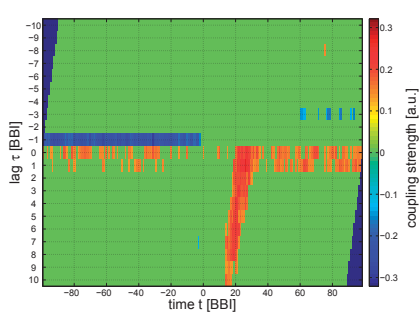

(b) BBI-DBP

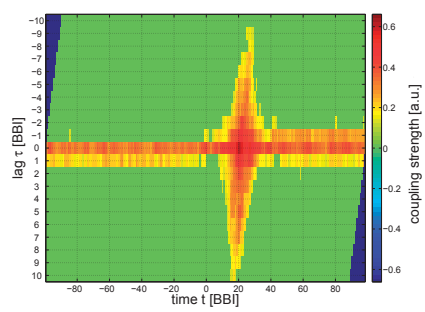

(e) SBP-DBP

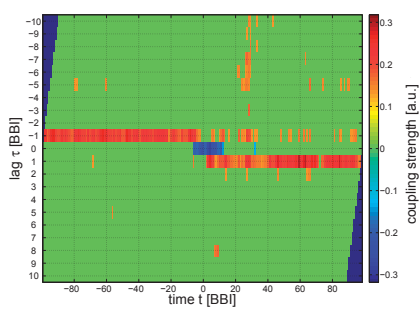

(h) DBP-SV

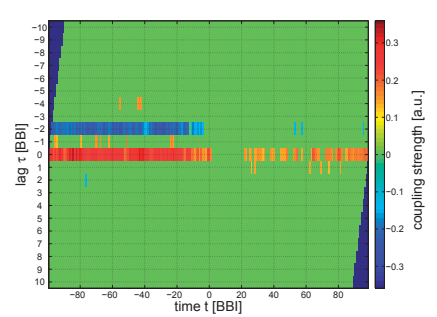

(c) BBI-SV

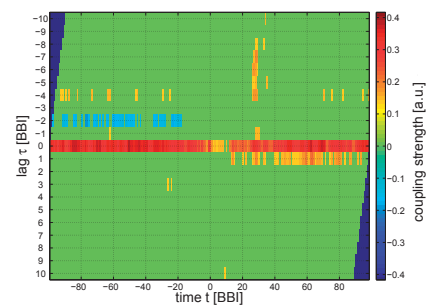

(f) SBP-SV

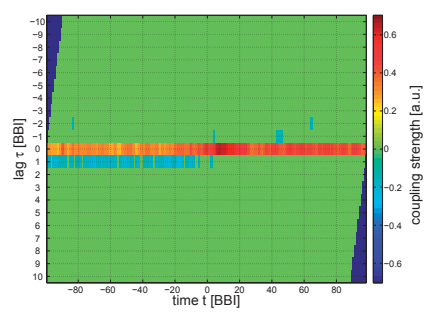

(j) SV-CO

Figure 4.3: This figure shows the resulting coupling structures from the ESCT analysis between the regarded cardiovascular variables during an orthostatic test. 
Due to the even smaller ensemble size $(\bar{M} \approx 86)$ the significance test is omitted this time. The results are shown in figures $4.4-4.7$. At a first glance, the reaction on the orthostatic test is in the main very similar. However, differences between the four groups can be detected in the relative coupling strengths. Generally, a reduced strength in the older subjects can be seen. The most prominent differences occur in the effect of the orthostatic test on the connection between the diastolic blood pressure and the stroke volume in the male and the younger subjects as compared to the other groups. 


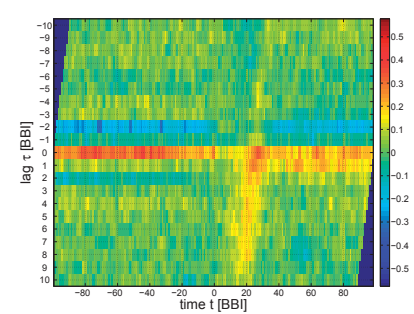

(a) BBI-SBP

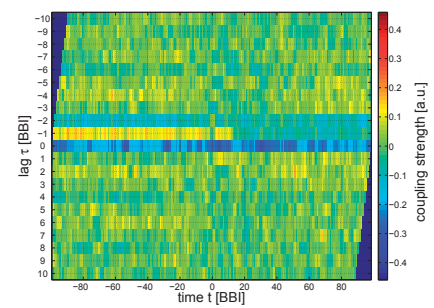

(d) BBI-CO

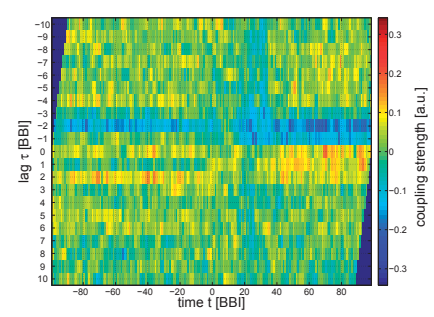

(g) SBP-CO

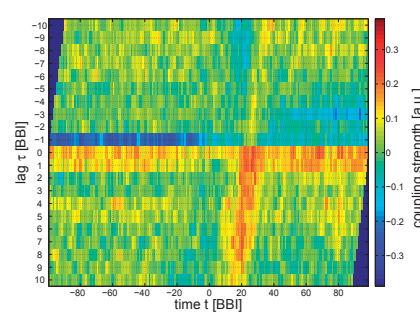

(b) BBI-DBP

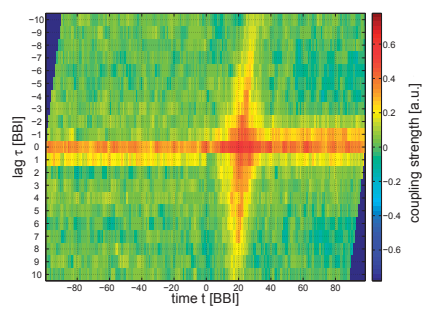

(e) SBP-DBP

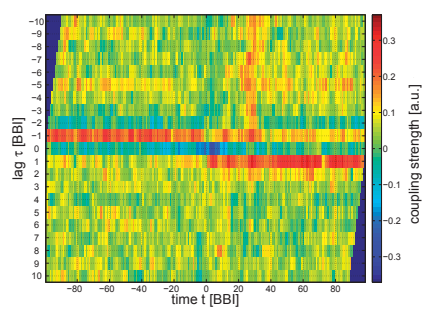

(h) DBP-SV

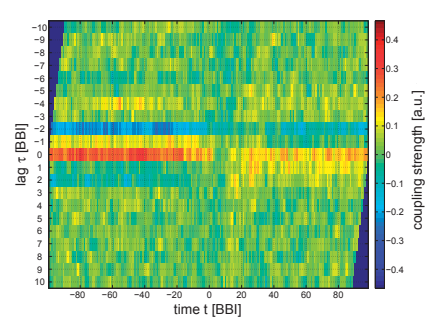

(c) BBI-SV

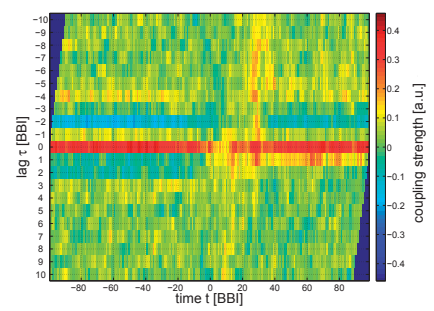

(f) SBP-SV

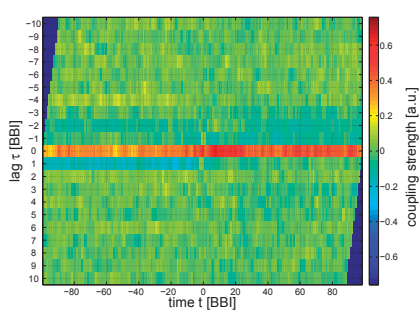

(j) SV-CO

Figure 4.4: This figure shows the ESCT results for the coupling structure between the cardiovascular variables regarded for the females group. 


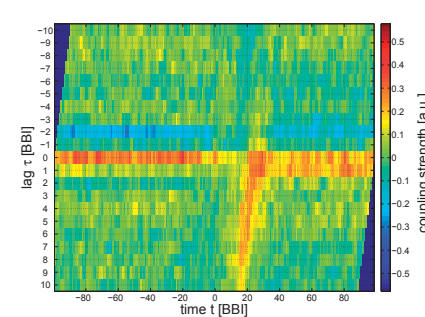

(a) BBI-SBP

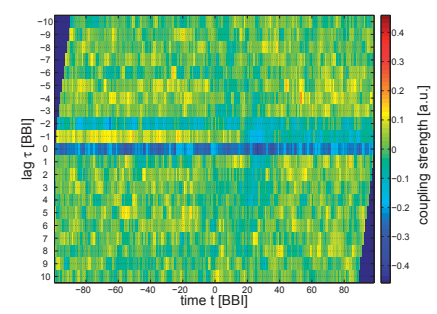

(d) BBI-CO

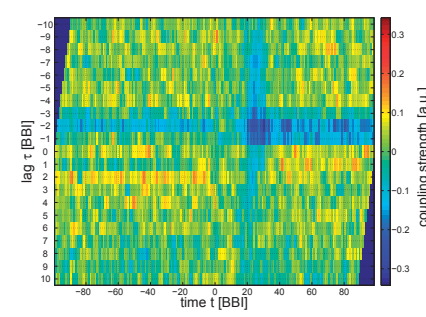

(g) SBP-CO

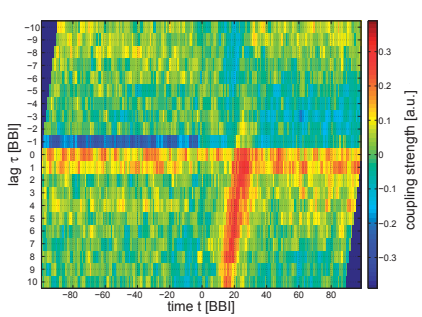

(b) BBI-DBP

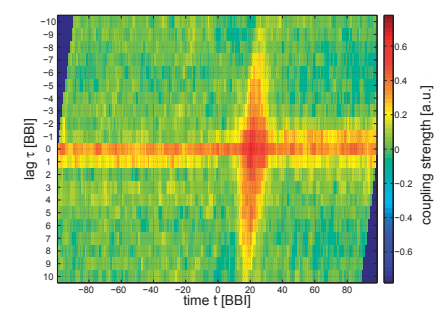

(e) SBP-DBP

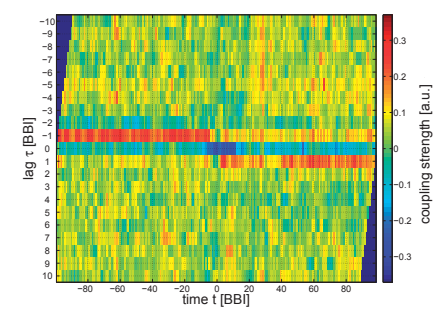

(h) DBP-SV

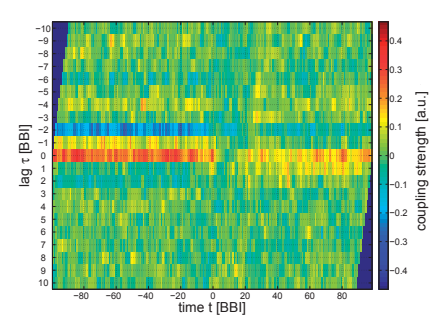

(c) BBI-SV

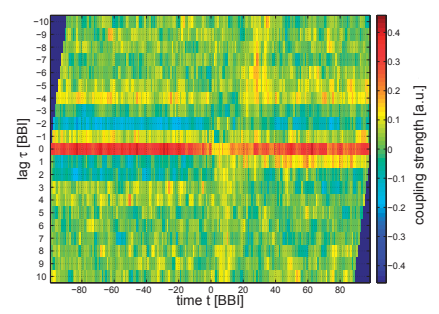

(f) SBP-SV

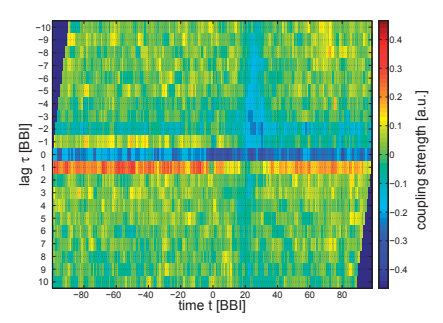

(i) DBP-CO

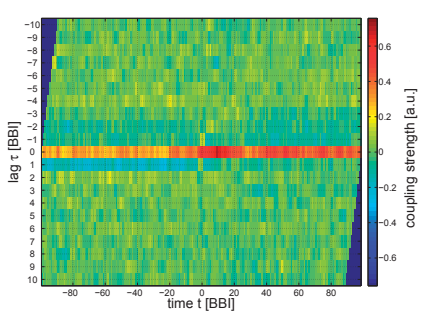

(j) SV-CO

Figure 4.5: This figure shows the ESCT results for the coupling structure between the cardiovascular variables regarded for the males group. 


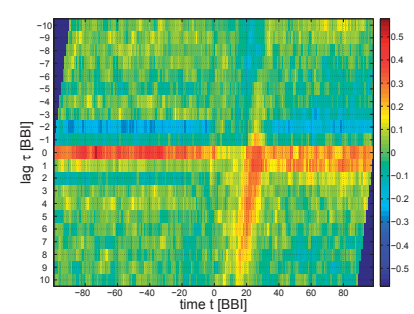

(a) BBI-SBP

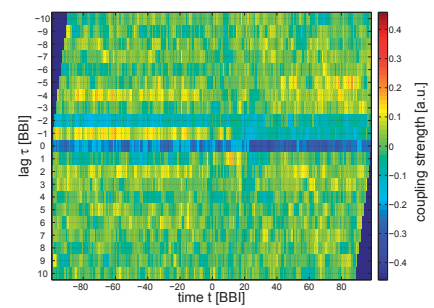

(d) BBI-CO

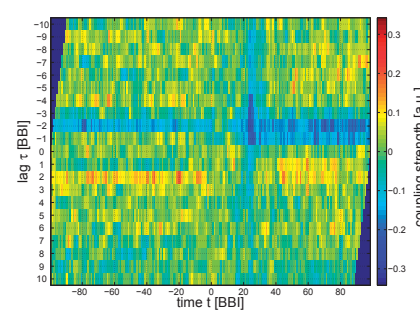

(g) SBP-CO

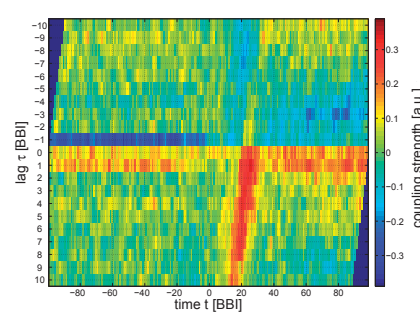

(b) BBI-DBP

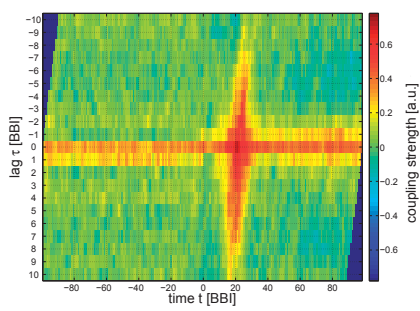

(e) SBP-DBP

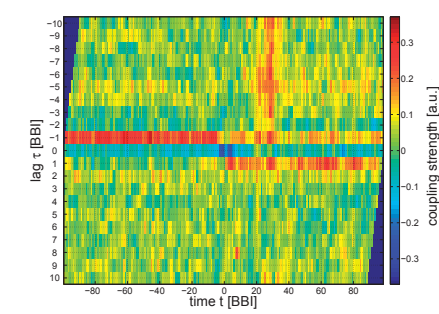

(h) DBP-SV

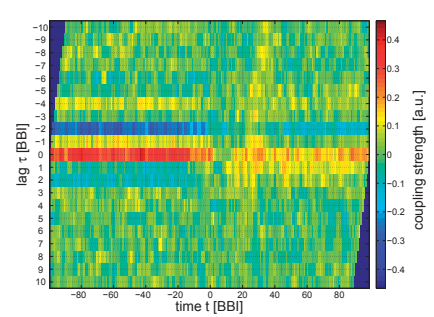

(c) BBI-SV

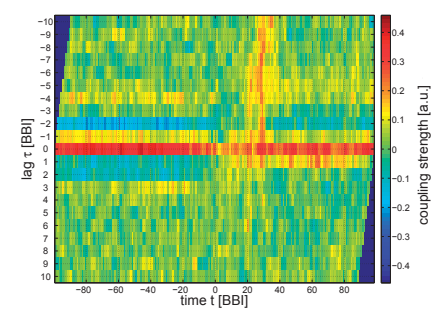

(f) SBP-SV

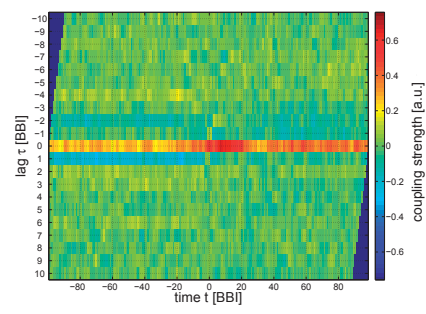

(j) SV-CO

Figure 4.6: This figure shows the ESCT results for the coupling structure between the cardiovascular variables regarded for the younger ages group. 


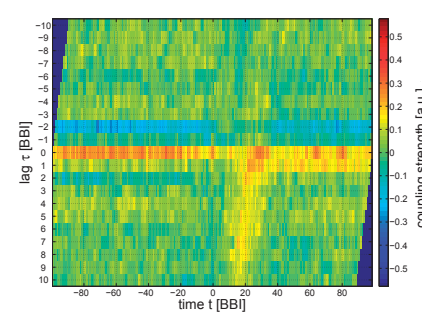

(a) BBI-SBP

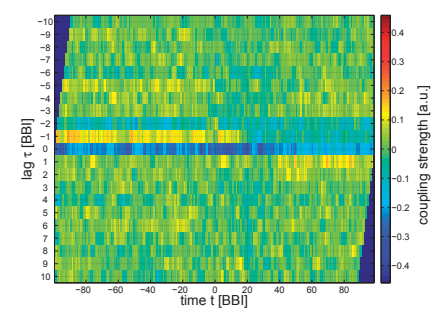

(d) BBI-CO

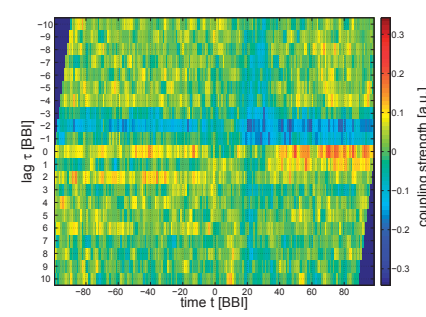

(g) SBP-CO

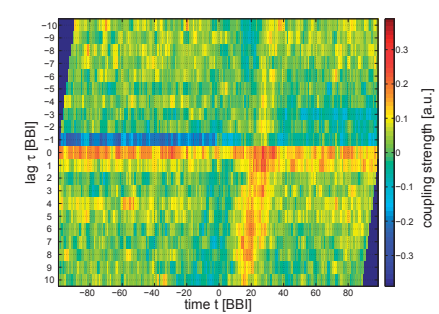

(b) BBI-DBP

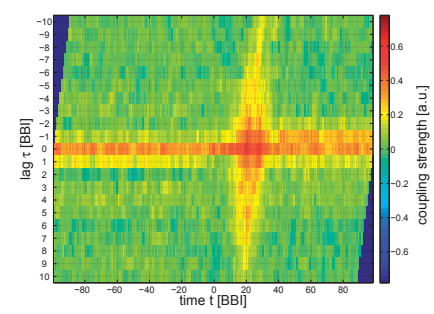

(e) SBP-DBP

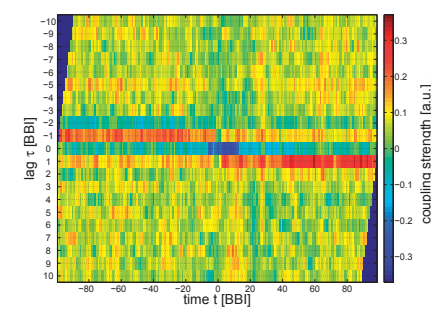

(h) DBP-SV

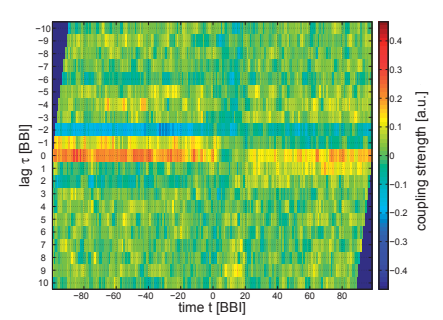

(c) BBI-SV

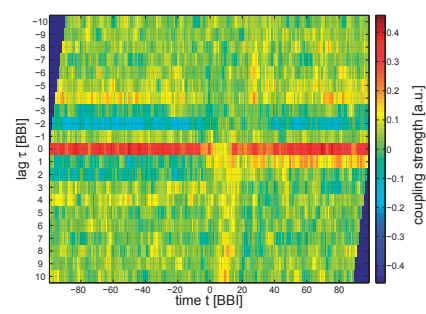

(f) SBP-SV

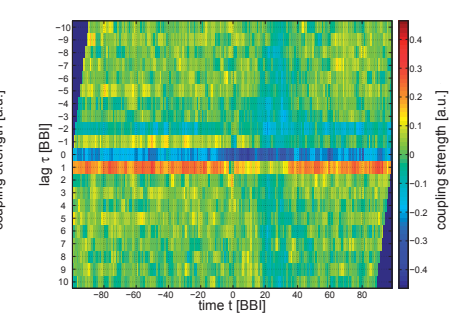

(i) DBP-CO

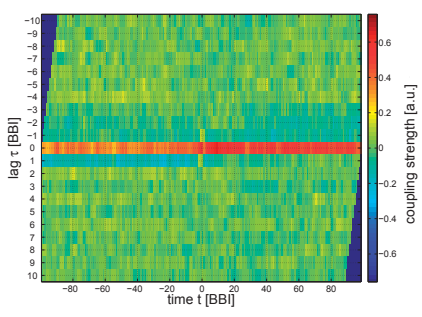

(j) SV-CO

Figure 4.7: This figure shows the ESCT results for the coupling structure between the cardiovascular variables regarded for the older ages group. 
4 Application to data measured during an orthostatic test

\subsection{Discussion}

During an orthostatic manoeuvre, the change from a supine to a standing position, blood is redistributed and the cardiovascular system has to cope with the effects. The blood rushing into the legs leads to a reduction of central venous pressure and thus to a decrease in stroke volume and systolic blood pressure. This again results into the activation of several regulative mechanisms like vasoconstriction, the contraction of arterial and venous blood vessels, a rise of the heart rate, and the release of certain hormones. The aim of these actions is the increase of the total peripheral resistance and the available blood volume in order to normalise the blood pressure levels [HW03].

The coupling analysis of the BBI and blood pressure data recorded during the orthostatic test (see figure 4.3 (a), (b), and (e)) confirms the predominating picture about cardiovascular shortterm regulation. The significant connections found in the stationary part of the data are from diastolic to systolic blood pressure (symmetric, $\tau=1$ ), depicting the Frank-Starling mechanism [Lev10, Sta18], the dependence of the ejected blood volume during the systole on the blood filling of the heart after the previous diastole, and from beat-to-beat intervals to systolic blood pressure (diametric, $\tau=-2$ ), illustrating the sympatho-vagal feedback via vasoconstriction and vasodilation due to respiratory movements $\left[\mathrm{WSR}^{+}\right.$09]. This depicts an anti-Starling effect, where an increase in heart rate also leads to an increase in diastolic and systolic blood pressure and vice versa. With just the regular Starling mechanism, an increase in heart rate would lead to a decrease in stroke volume, since the filling time of the ventricles would reduced, and thus to a decrease in blood pressure. This effect, however, seems to be smaller than the influence of the total peripheral resistance, which is why only the latter effect is observed here. The lag $\tau=0$ connections between diastolic blood pressure and beat-to-beat intervals (diametric) and between beat-to-beat intervals and systolic blood pressure (symmetric) represent mechanically induced arterial pressure fluctuations due to respiratory movement $\left[\mathrm{WSR}^{+} 09\right]$ while the fluctuations in the coupling strength hint at the respiratory sinus arrhythmia, i.e. the change in heart rate when breathing in or out.

The coupling structure found in the nonstationary part during the orthostatic manoeuvre beginning at $t=0$ further corroborates the opinions about the short-term regulation. The growing symmetric influence of blood pressure on the beat-to-beat intervals can be identified with the sympathetic baroreflex trying to compensate the drop in blood pressure. The gap in the $\tau=-2$ interaction between beat-to-beat intervals and the systolic blood pressure can be explained by the attenuated respiratory sinus arrhythmia and the strong dominance of the blood pressure drop at this time. The fluctuations in the coupling strength show an adapting process between blood pressure and heart rate, as the minima and maxima in the $\tau=0$ and $\tau=1$ region are alternating, indicating the presence of fast vagal and slower sympathetic controls. 
An interesting result is found in the relationship between the diastolic blood pressure and the stroke volume (figure $4.3(\mathrm{~h})$ ). Before the event the symmetric influence of the DBP on the SV at $\tau=-1$ again depicts the well-known Frank-Starling law of the heart [Lev10, Sta18], telling us that the blood volume ejected from the heart during one heart beat depends on the diastolic pressure during the previous cycle. After an initial decrease in SV, about ten heart beats after the event, the SV increases slightly (diametric effect at $\tau=0$ ) before dropping further. This temporal rise of SV, which cannot be found in the other variables regarded together with the $\tau=0$ lag hints at other variables influencing the SV. One of these is most probably the contractility, i.e. the intrinsic ability of the heart to contract, which increases e.g. with an increase in heart rate and sympathetic activation [Lev10]. This effect attenuates the effects of the drastic fall in diastolic pressure, but only for a short time. While the other variables regarded here are almost back to normal values after about 40 BBIs following the orthostatic test, the SV values stay very low. This might be due to a refractory period in the contractility increasing effects regulated via hormone releases.

An often used model of the cardiovascular system on a beat-to-beat basis, the DeBoer-model, was introduced in [DKS87]. It is able to quantitatively describe power- and cross-spectra of heart rate and blood pressure variability, incorporating meachnisms like the baroreflex as well as respiratory influences. Apart from simulating subjects at rest it is also suited for simulating data that would be measured after the application of vasoconstricting drugs. A comparison of the coupling analysis results gotten from the measured data with the DeBoer-model with a simulated orthostatic test shows, that there are more mechanisms at work than already incorporated in the model. The drop in systolic blood pressure only leads to a short disruption in the stationary coupling structure in the model, without the drastic effects on the other variables. However, the average time of the disruption in the model and the outburst in the measured data lies in both cases around 30 to 40 heart beats. In the model, after that amount of time the system is back to the normal state, while the measured data shows longer-lasting effects. There are already models specialising in the transient behaviour of cardiovascular variables during an orthostatic test. In [HSKM02] a continuous hemodynamic model is introduced that already shows promising results at least in the response of the heart rate during head-up-tilt. Of course, here one has to keep in mind the differences between orthostatic tests performed by simply standing up (as in the data used in this work) and tests employing a tilt table. The head-up-tilt in the latter case would show even more drastic results since the attenuating effect of muscle contraction when standing up falls away.

The analysis of the different groups, showed differences in the coupling structures depending on gender and age. Mainly because of the smaller ensemble sizes, the differences between the groups are not significant. Therefore, a purely qualitative interpretation of the differences 
will be given here. In general the older group displays a weaker effect in the coupling structure during the event (compare e.g. figures 4.6-4.7 (a), (b), and (e)). After the orthostatic test event there are different effects depending on the variables regarded. For the older group there is a stronger lingering effect in the relationship between systolic and diastolic blood pressure than in the younger one (figures 4.6-4.7 (e)) and between the stroke volume and the cardiac output (figures 4.6-4.7 (j)) while the other pairings of variables show a weaker reaction. This hints at a higher stiffness of the blood vessles with older age and thus at a reduced adaptability to blood pressure changes. Regarding gender effects, the clearest differences can be found looking at figures 4.4-4.5 (b) and (h). In the first case, men show a stronger reaction in the symmetric influence of DBP on BBIs directly following the orthostatic test, while in the second case the men show a weaker coupling from SV to DBP compared to the other groups.

The weaker reaction following the orthostatic test and the stronger lingering effects for the older subjects confirm studies reporting a reduced adaptability and variability in cardiovascular variables for higher ages $\left[\mathrm{BKO}^{+} 08, \mathrm{BOL}^{+} 07, \mathrm{BWB}^{+} 03, \mathrm{KLY}^{+} 99\right.$, OS03]. Studies analysing the effect of orthostatic tests with regard to gender differences reported a higher frequency of orthostatic dysfunction in women. However, there are often conflicting results, making it difficult to formulate conclusive interpretations $\left[\mathrm{BKO}^{+} 08\right]$. Following the assumption of a higher chance of orthostatic dysfunction in women, the stronger effect in figures 4.4-4.5 (b) for men might hint at a better adaptability to drop in blood pressure. Also the weaker coupling from DBP on SV for the younger group points at faster adaptive mechanism which is lost with higher age. The similarity between the coupling structures from the older and the female group also hints at the of vanishing gender differences with higher ages, confirming previous studies $\left[\mathrm{BWB}^{+} 03, \mathrm{KLY}^{+} 99\right]$. A distinction between younger / older female / male groups further corrobates this finding, although this encompasses a further reduction in ensemble size and thus gives even less meaningful results.

The application of the ESCT is able to disclose and confirm stationary as well as nonstationary effects of the orthostatic test onto short-term cardiovascular control. This allows for a more accurate analysis of the mechanisms involved. Studies on bigger groups, separated by healthy and sick subjects, BMI, or other factors, will give even deeper insights on the functions and interactions of cardiovascular variables during such a test. 


\section{Application to data measured during sleep arousals}

In this chapter a data set comprising two measurement nights from one subject displaying a large number of arousals is analysed. This case belongs to the second group of recoveries after disturbances and the ensemble is built up from several repetitions of one event from one subject.

\subsection{Analysis}

The data to be analysed (see table 5.1 in this subsection stems from a study [PRG ${ }^{+} 12$ ], where 27 patients suffering from obstructive sleep apnoea syndrome were measured for two nights. The measurement comprises an ECG, an electroencephalogram, an electrooculogram, various respiratory signals, electromyograms from chin and legs, as well as a continuous blood pressure measurement. Sleep stages have been scored according to the standard AASM manual [IAICQ07]. From the ECG the R-peaks were detected to build the BBI series and systolic and diastolic blood pressure have been extracted. The other signals have been preprocessed by determining mean and variance during the BBIs. One of the subjects showed a particularly high occurrence of arousals during the two nights. The typical characteristics of these arousals in the regarded cardiovascular variables can be seen in figure 5.1 where the normalised time series averaged across the ensemble are shown.

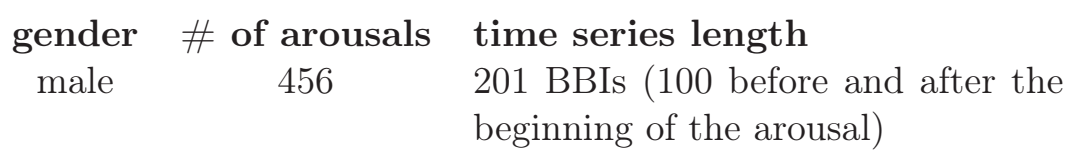

Table 5.1: The table shows information about the sleep data measurement.

The arousals have been marked in the BBI series and their beginning $(t=0)$ has been used to align the ensemble members. Due to the restricted ensemble size $(M=456)$ and natural noise, the significance level has been set to $\alpha=0.2$ (as before there was no discernible structure in the results for $\alpha=0.05$ ) while the model order was chosen as $\Omega=10$ following the BIC. The suggested model order according to the AIC was $\Omega=6$. As in the previous subsection, the clearest results are given by the ESCT-method. In figure 5.2 the resulting significant coupling structures according to the ESCT are shown between the variables of BBI, SBP, DBP, as well 


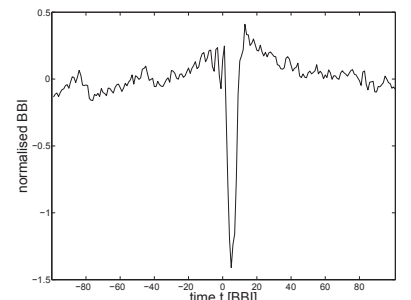

(a) BBI

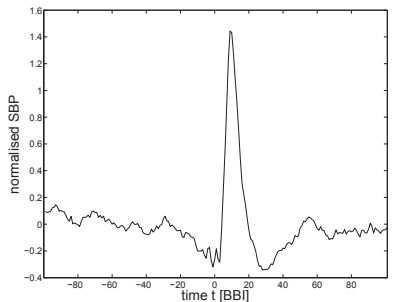

(b) SBP

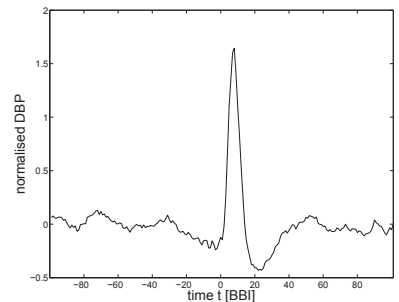

(c) DBP

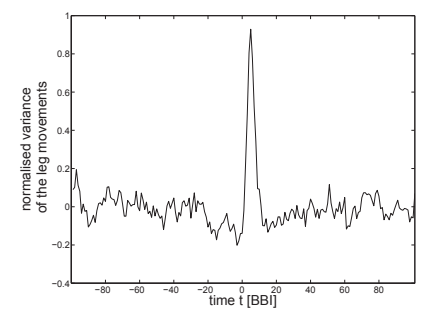

(d) Leg movement

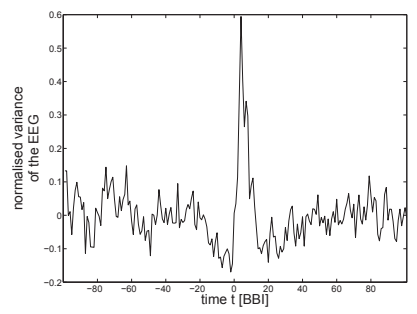

(e) EEG

Figure 5.1: This figure shows an example of the typical form of arousals in the BBI-series (a) frequently occurring in one subject from the study described in $\left[\mathrm{PRG}^{+} 12\right]$ during the measurement nights. The effect of the arousals in the other variables regarded (b)-(e) is also shown.

as the activity, i.e. the windowed variance during one beat-to-beat interval, of the left leg and the EEG. The used EEG-channel was the lead from electrode positions O2 (right occipetal lobe) and A1 (left ear lobe). Again, 100 BBIs before and after the alignment point have been regarded in the analysis, but for a better representation figures 5.2 (d)-(f) are shown only with ten BBIs before and after the event.

Here the known stationary structure between BBI, SBP and DBP is disrupted, as in the orthostatic test, during the arousal (compare figures 5.2 (a)-(c)). The $\tau=-2$ diametric influence from the BBI onto the SBP is getting stronger and spreading to larger lags. After about ten heartbeats the influence changes into symmetric behaviour, before, after about 20 heartbeats, the original structure is restored. The same effect can be regarded between the BBI and the DBP series. The coupling between the DBP and the SBP series shows a stationary $\tau=-1$ influence from the DBP to the SBP. The effects of the events show an almost symmetric distribution across several lags $(\tau<0$ and $\tau>0)$. In the other three figures 5.2 (d)-(f) no coupling could be discerned before the event. Only during and directly after the event a short occurrence of significant lags can be seen. At first a diametric influence from the variance of the leg movement onto the beat-to-beat intervals can be seen which is reversed into a symmetric influence 


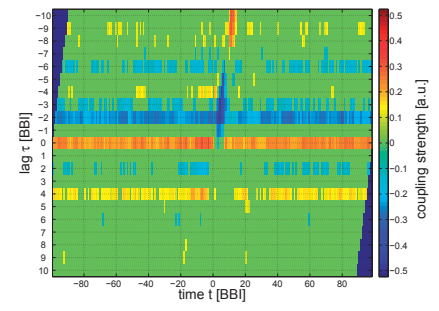

(a) BBI-SBP

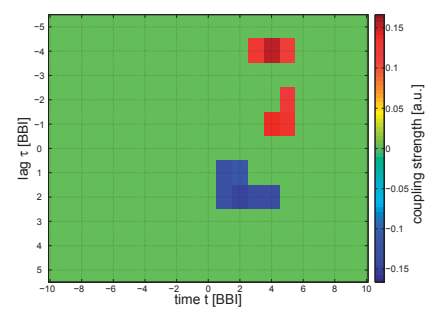

(d) BBI-Leg movement

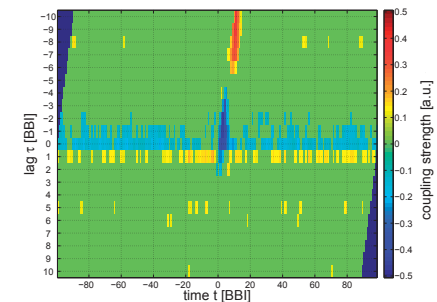

(b) BBI-DBP

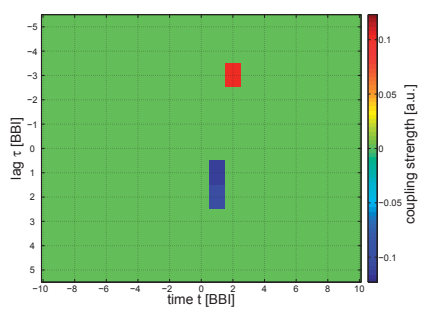

(e) BBI-EEG

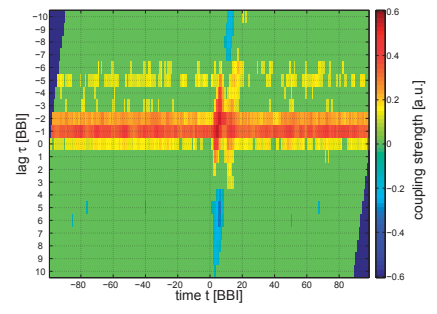

(c) DBP-SBP

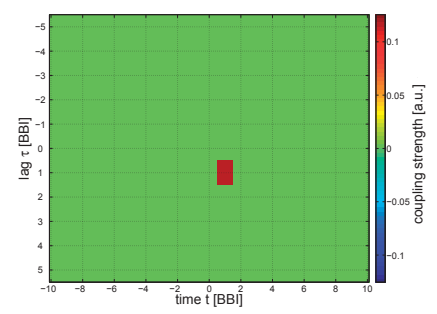

(f) Leg-Movement-EEG

Figure 5.2: This figure shows the ESCT coupling structure between the regarded variables during the arousals frequently occurring in one subject from the study described in $\left[\mathrm{PRG}^{+} 12\right]$ during the measurement nights.

from the BBI onto the leg movements after three to four heart beats. A similar pattern can be discerned when looking at the relationship between the BBIs and the variance of the EEG. Figure 5.2 (f) only shows a short effect from the EEG onto the leg movements.

\subsection{Discussion}

The results delivered by the ESCT-method for the polysomnographic data with the arousals at first also confirm the results mentioned above and from [3], [WSR $\left.{ }^{+} 09\right]$ about the short-term cardiovascular regulation between heart rate and blood pressure. Again the effects originating in the sympatho-vagal feedback (figure $5.2(\mathrm{a})$, diametric $\tau=-2$ ), the Frank-Starling mechanism (figure $5.2(\mathrm{c})$, symmetric $\tau=-1$ ), and in the mechanical influence of the respiratory movements (figure $5.2(\mathrm{a})-(\mathrm{c}), \tau=0$ ) are clearly visible. With the beginning of the arousals at $t=0$ the heart rate increases at first, followed by an increase in the blood pressure (diametric effect in figures 5.2 (a) and (b)). About five heart beats later the heart rate goes down again while the blood pressure is still rising (symmetric effect). This corresponds to the baroreflex, which regulates sympathetic and parasympathetic activity depending on the current blood pressure and thus influences the heart rate. Via figures 5.2 (d)-(f) further conjectures about the cause 
of the arousals can be made. Approximately two heart beats before the length of the BBIs shortens, a rise in the strength of the leg movements and in the brain activity can be seen (diametric effect). But again, the heart rate goes down before the activity in the leg movements and the EEG normalises. Figure 5.2 (f) shows that the increased leg movement is triggered by the rise in the brain activity with a time lag of $\tau=1$ (symmetric effect). Thus, we can see that the arousal is triggered in the brain leading first to increased leg movement via motor activation and then due to sympathetic activation to a rise in heart rate, which again induces the blood pressure fluctuations.

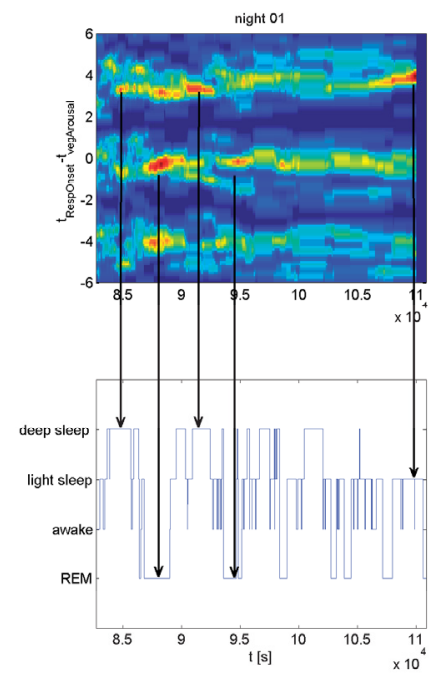

(a)
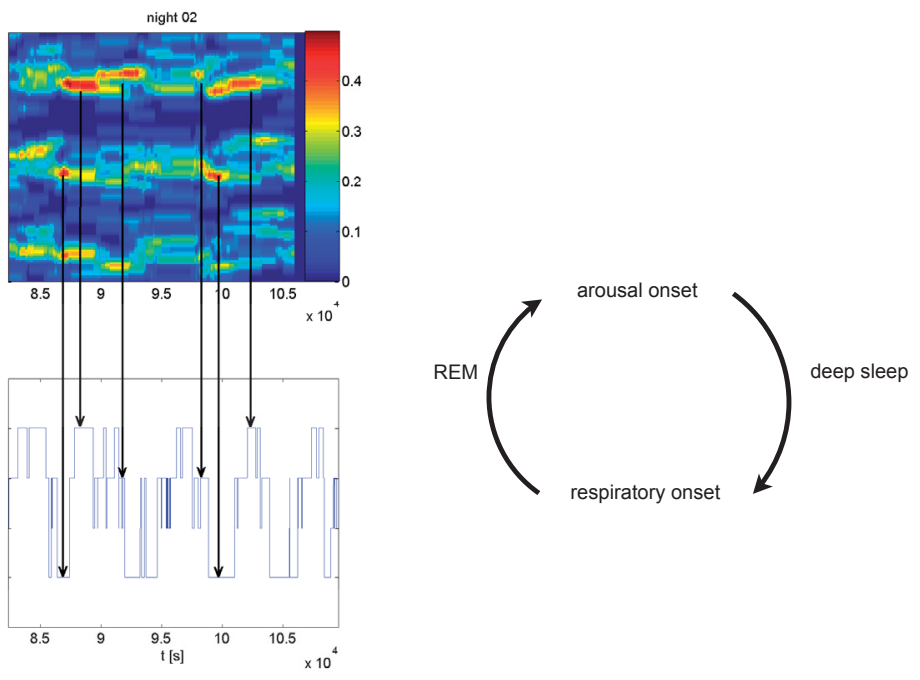

(b)

Figure 5.3: In (a) the Coordigram between respiratory and arousal onsets during the different sleep stages in one subject from the study described in $\left[\mathrm{PRG}^{+} 12\right]$ during the two measurement nights is shown. The diagram in (b) summarises the information inferred from the coordigram.

Another tool, the so-called coordigram, allowing for a coupling analysis with a very high temporal resolution was introduced in [6]. It has been used to analyse the relationship between the heart beats and the onsets of the respiratory cycles. The coordigram's ability to work with very few time points allows for an analysis of the differences of the arousals' effects during different sleep stages. In this special case not the interrelation between the heart beats and the onsets of the respiration, but the coordination between the beginnings of the respiratory cycles and the onsets of the vegetative arousals has been analysed. In [4] it was found, that the direction of that interrelation is dependent on the sleep stage. During deep sleep the 
arousals seem to control the beginning of the following respiratory movement (figure 5.3). The time distance between arousal onset and the beginning of the expiration is too long for an autonomic reaction, suggesting instead a control of the onset of the inspiration. This in turn would result in a time delay between the onsets of one heart beat. This effect shows similarities to the trigger effect of single heart beats onto the beginning of the respiratory cycle described in [Ras87]. This hints at a connection between the cardiac centre and the respiratory generators in the brain stem. During the rapid-eye-movement (REM) sleep the direction of the interaction is reversed (figure 5.3). This time the time delay suggests a relation between the onset of the expiratory phase and the arousal beginning, which might be due to a change of the respiration generating process. During REM-sleep higher brain regions dominate the respiratory rhythm [Rem05]. So in this case the former interrelationship is destroyed or overlapped since there is no connection between the cardiac centre and the higher brain areas. In conclusion, the coordigram analysis enhances the results gotten via the ESCT. 


\section{Conclusion \& Outlook}

In this work, a group of well-known and often applied coupling measures has been extended to allow for a time-resolved coupling analysis with a very high temporal resolution. The extension is based on the usage of repeated events to build an ensemble over which the regarded measures are to be estimated. The time resolution to be achieved with this approach is only dependent on the measures themselves and goes up to a time span comprising only a few time points of the measured data. This enables us to analyse transient events e.g. in climate, financial, or cardiovascular time series. The only requirement for the ensemble approach to work is the repeated occurence of such events, either measured in different subjects or as repetitions in one subject.

\section{Methods}

The tools regarded in this thesis are the ensemble cross correlation (subsection 2.2.3), the ensemble lagged mutual information (subsection 2.2.3), the ensemble partial Granger causality (subsection 2.3.4), and the ensemble symbolic coupling traces and permutation symbolic coupling traces (subsection 2.4.3). They were chosen to represent the majority of fields modern coupling measures stem from, namely classical methods, information theory, model-based tools, and symbolic dynamics. For each method the basics of how they work as well as the ideas behind have been given and explained before they were extended using the ensemble approach. The code for the EGC, the PESCT, and the ESCT for MATLAB (MATLAB and Statistics Toolbox Release 2012a, The MathWorks, Inc., Natick, Massachusetts, United States) can be found at http://tocsy.pik-potsdam.de/coupling.php.

\section{Models}

All measures introduced and extended in this work have been extensively tested on several theoretical models to assess their performance on linear (subsection 3.1.1) and nonlinear systems (subsection 3.1.2), their behaviour in the presence of noise, for different ensemble sizes, and for different coupling strengths (subsection 3.1.3). The results showed that the SCT methods are suitable for a lot of applications, due to their model-free approach, their robustness against noise, and their low requirements in terms of the length of the data series. A drawback of these methods is their bivariate nature. Another flexible approach is given by the Granger causality, which can be customised by using models best suited to the data in question. There are already a lot of extensions [AMS04, CRFD04, HSPVB07, IOLK08a, IOLK08b, FNC08, 
Rie09, $\mathrm{RSM}^{+}$08, $\mathrm{RSS}^{+}$10] allowing for multivariate and nonlinear analyses. But to apply the most suitable version of Granger causality one has to have some a priori knowledge about the system at hand. With more data available refined versions of the lagged mutual information [VP08], [9] are also an option because of their inherent ability to cope with highly nonlinear systems and couplings. Multivariate extensions exist and usually no further a priori knowledge about the data is necessary.

\section{Orthostatic test and sleep arousals}

In chapters 4 and 5 two examples of transient events in the cardiovascular system have been analysed. The results show interesting consequences of these events for the regarded variables and help us to get a deeper insight into the system and the cardiovascular control mechanisms. The different tests and the application to measured data proved the ESCT method to be an effective low-cost tool for a first assessment of the existing coupling structure.

In the case of the orthostatic test (chapter 4) it was possible to identify the stationary coupling structure between BBIs, systolic and diastolic blood pressure, stroke volume, and cardiac output before the event. The couplings show the sympatho-vagal feedback, the baroreflex, and the Frank-Starling mechanism while fluctuations in the coupling strengths show for example the effects of the respiratory sinus arrhythmia. During and after the event the different behaviours during the nonstationary part can clearly be seen. The results confirm predominant opinions about the processes during an orthostatic manoeuvre by showing mechanic and hormonal effects which are usually not visible by employing standard methods. Comparisons by gender and age were limited by the amount of data available but still go according with the commonly accepted view of a reduced adaptability with higher ages as shown in stonger lingering effects of the manoeuvre. Also vanishing gender differences with higher age can be confirmed, with men showing a higher adaptability at younger ages than women.

The findings for the sleep arousal data (chapter 5) again corroborate existing results for the stationary couplings and show the known short-term cardiovascular regulation mechanisms. The nonstationary part allows speculation about the chain of events during the course of the arousals. Apart from the baroreflex, which regulates sympathetic and parasympathetic activity depending on the current blood pressure, being clearly visible, we can see the faster motor activation followed by the sympathetic activation leading to the increases in leg movements and heart rate. These are triggered in the brain [4]. With the help of the coordigram a reversal of the connection between arousal beginnings and respiratory onsets in deep sleep as opposed to REM sleep has been found. This allows further assumptions being drawn about a connection between the cardiac centre and the respiratory generators $[4,6]$. 
6 Conclusion \& Outlook

\section{Outlook}

Of course, this work is far from exhaustive concerning the field of coupling analyses as there are many other coupling measures not regarded here. From these especially the ones stemming from information theory [VP08], [9] show promise at identifying actual causalities via information transfer, while excluding spurious couplings mediated by other variables present. However, these usually require very large amounts of data and are thus not always suitable for an ensemble analysis on real-life data, where the number of available realisations is often limited.

Future work should comprise the extension and testing of other coupling measures to enable the analysis of transient events in areas where the ESCT and linear Granger causality fail. The biggest problems lying here in multidimensional nonlinear systems. Furthermore, the ensemble approach requires the repetition of the events of interest. So, the application to bigger data sets would offer more flexibility in building the ensembles and thus allow for comparisons depending on e.g. BMI, subjects with and without certain diseases, and other factors. Also algorithms for automatic group building could then be employed. However, the ensemble approach is not always possible to realise. In this case a windowed analysis might still be the method of choice and tools like the coordigram [6] can offer new and interesting approaches.

In the end, the ensemble coupling analysis methods offer an effective, easily applicable way to learn a systems reaction under very short but extreme events, allowing for a better understanding of the system's inner structure. This, in turn, can be used to devise new ways to cope with e.g. financial shocks, climate changes, and cardiovascular diseases, and thus to help to treat and solve current and future problems from many different fields. 


\title{
List of Abbreviations
}

\author{
AIC .......... Akaike information criterion \\ AR .......... autoregressive \\ BBI .......... beat-to-beat interval \\ BIC .......... Bayesian information criterion \\ BPV ......... blood pressure variability \\ DBP ......... diastolic blood pressure \\ CO $\ldots \ldots \ldots \ldots$ cardiac output \\ ECG $\ldots \ldots \ldots$ electrocardiogram \\ EEG ......... electroencephalogram \\ EGC ......... ensemble Granger causality \\ EJT $\ldots \ldots \ldots$ ejection time
}

ESCT $\ldots . . \ldots$ ensemble symbolic coupling traces

EMI ......... ensemble mutual information

EXCORR .... ensemble cross-correlation

HRV ......... heart rate variability

NAAR ........ nonlinear additive autoregressive

NAARX ...... nonlinear additive autoregressive with external input

PESCT ...... permutation ensemble symbolic coupling traces

SBP systolic blood pressure

SCT $\ldots \ldots \ldots$ symbolic coupling traces

SV $\ldots \ldots \ldots$...... stroke volume 


\section{List of Frequently used mathematical symbols}

$\alpha$ significance level

$\operatorname{Cov}(\cdot, \cdot) \ldots \ldots$. covariance

$\epsilon \ldots \ldots \ldots \ldots \ldots$ independent white noise

$\varepsilon \ldots \ldots \ldots \ldots$ error term, residuals

$M, m \ldots \ldots \ldots$ \# ensemble members, ensemble index (might refer to the whole ensemble)

$N \ldots \ldots \ldots \ldots$ number of time points in data series

$P, p \ldots \ldots \ldots \ldots$ probability, probability distribution

$s(t) \ldots \ldots \ldots \ldots$ symbol series

$\sigma, \Sigma \ldots \ldots \ldots \ldots$ standard deviation, covariance matrix

$\operatorname{sgn} \ldots \ldots \ldots \ldots$ signum function

$t, \tau \ldots \ldots \ldots \ldots$ time (usually refers to the whole vector, e.g. $t=1, \ldots, N$ ), time lag

$\operatorname{Var}(\cdot) \ldots \ldots \ldots$. variance

$W, w(t) \ldots \ldots .$. word, word series

$X, Y, Z \ldots \ldots$ systems or processes

$x(t), y(t), z(t) \ldots$ time series

$\omega, \Omega \ldots \ldots \ldots \ldots$ orders for models and Granger causality 


\section{List of Figures}

2.1 Time vs. ensemble average . . . . . . . . . . . . . . . . . . . . . . . . . . . 19

2.2 Graph for time resolved coupling measures . . . . . . . . . . . . . . . . . 20

2.3 Permutation patterns . . . . . . . . . . . . . . . . . . . . . . . . . 31

3.1 Coupling structure results for $x_{1}, x_{2}$ of the first AR-process $\ldots \ldots \ldots$. . . . 37

3.2 Coupling structure results for $x_{4}, x_{5}$ of the first AR-process $\ldots \ldots \ldots$

3.3 Coupling structure first AR-process . . . . . . . . . . . . . . . . 38

3.4 Coupling structure results for $x_{1}, x_{2}$ of the second AR-process . . . . . . . . 40

3.5 Coupling structure second AR-process . . . . . . . . . . . . . . . 40

3.6 Coupling structure results for $x_{1}, x_{2}$ of linearly coupled logistic maps . . . . . . 43

3.7 Coupling structure results for $x_{1}, x_{3}$ of linearly coupled logistic maps . . . . . 43

3.8 Coupling structure results for $x_{2}, x_{3}$ of linearly coupled logistic maps . . . . . . 44

3.9 Coupling structure of the linearly coupled logistic maps . . . . . . . . . . . 44

3.10 Coupling structure results for $x_{1}, x_{2}$ of nonlinearly coupled logistic maps . . . . 46

3.11 Coupling structure of the nonlinearly coupled logistic maps . . . . . . . . . 46

3.12 Coupling structure results for $x_{1}, x_{2}$ of the NAARX-model . . . . . . . . 48

3.13 Coupling structure NAARX-model . . . . . . . . . . . . . . . . 49

3.14 Quality of the results depending on the ensemble size . . . . . . . . . . . 51

3.15 Quality of the results depending on the coupling strength . . . . . . . . . 51

3.16 Quality of the results depending on the magnitude of additive noise . . . . . . 52

4.1 Cardiovascular variables during orthostatic test f . . . . . . . . . 56

4.2 Coupling structure BBI-SBP orthostatic test . . . . . . . . . . . . 57

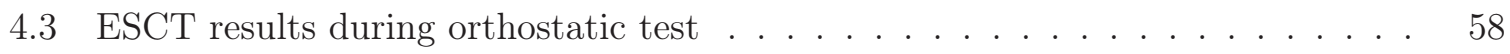

4.4 ESCT results during orthostatic test for the female subjects . . . . . . . . . 60

4.5 ESCT results during orthostatic test for the male subjects . . . . . . . . . . . 61

4.6 ESCT results during orthostatic test for the younger subjects . . . . . . . . . 62

4.7 ESCT results during orthostatic test for the older subjects . . . . . . . . . . . 63

5.1 Arousal in the the regarded variables . . . . . . . . . . . . . . . 68

5.2 ESCT results during arousals . . . . . . . . . . . . . . . . . . . 69

5.3 Coordigram arousals . . . . . . . . . . . . . . . . . . . 70 


\section{List of Tables}

2.1 Building SCT . . . . . . . . . . . . . . . . . . . . . 29

3.1 AR-model with five variables . . . . . . . . . . . . . . 36

3.2 AR-model with multiple lags . . . . . . . . . . . . . . . . . 39

3.3 Linearly coupled logistic maps . . . . . . . . . . . . . . . . . . . . . . . . . . . . . 42

3.4 Nonlinearly coupled logistic maps . . . . . . . . . . . . . . . . . . 45

3.5 NAARX-model . . . . . . . . . . . . . . . . . . . . . . . 48

3.6 AR-model with two variables to test for robustness . . . . . . . . . . 50

4.1 Orthostatic test data . . . . . . . . . . . . . . . . 56

5.1 Sleep arousals data . . . . . . . . . . . . . . . . . . 67 


\section{List of Publications}

[1] Andreas Müller, Hendrik Bonnemeier, Hagen Malberg, Jürgen Kurths, and Niels Wessel. Age-dependent changes in the manifestations of gender-related differences in the cardiovascular regulation. In 8th Conference of the European Study Group on Cardiovascular Oscillations (ESGCO), pages 147-148. IEEE, 2014.

[2] Andreas Müller, Maik Riedl, Jan F. Kraemer, Thomas Penzel, Jürgen Kurths, and Niels Wessel. Symbolic Coupling Traces during arousals. In 48th annual conference of the German Society for Biomedical Engineering (BMT 2014), page 214, 2014.

[3] Andreas Müller, Maik Riedl, Thomas Penzel, Hendrik Bonnemeier, Jürgen Kurths, and Niels Wessel. Coupling analysis of transient cardiovascular dynamics. Biomedizinische Technik. Biomedical Engineering, 58(2):131-139, 2013.

[4] Andreas Müller, Maik Riedl, Thomas Penzel, Jürgen Kurths, and Niels Wessel. Kardiorespiratorische Koordination und Ensemble-Kopplungsspuren zur ereignisbasierten Charakterisierung kardiovaskulärer Interaktionen während des Schlafes. Somnologie - Schlafforschung und Schlafmedizin, 18(4):243-251, 2014.

[5] Andreas Müller, Maik Riedl, Niels Wessel, Jürgen Kurths, and Thomas Penzel. Methoden zur Analyse kardiorespiratorischer und kardiovaskulärer Kopplungen. Somnologie Schlafforschung und Schlafmedizin, 16(1):24-31, March 2012.

[6] Maik Riedl, Andreas Müller, Jan F. Kraemer, Thomas Penzel, Jürgen Kurths, and Niels Wessel. Cardio-Respiratory Coordination Increases during Sleep Apnea. PloS one, 9(4):e93866, January 2014.

[7] Maik Riedl, Andreas Müller, Jan F. Kraemer, Thomas Penzel, Jürgen Kurths, and Niels Wessel. Increased cardio-respiratory coordination in sleep apnoeas. In 48th annual conference of the German Society for Biomedical Engineering (BMT 2014), page 215, 2014.

[8] Maik Riedl, Andreas Müller, and Niels Wessel. Practical considerations of permutation entropy. The European Physical Journal Special Topics, 222(2):249-262, June 2013.

[9] Jakob Runge, Maik Riedl, Andreas Müller, Holger Stepan, Niels Wessel, and Jürgen Kurths. Quantifying the causal strength of multivariate cardiovascular couplings with momentary information transfer. In 8th Conference of the European Study Group on Cardiovascular Oscillations (ESGCO), pages 149-150, 2014.

[10] Niels Wessel, Maik Riedl, Jan F. Kraemer, Andreas Müller, Thomas Penzel, and Jürgen Kurths. Synchronisation and coupling analysis: applied cardiovascular physics in sleep medicine. In Annual International Conference of the IEEE Engineering in Medicine and Biology Society. IEEE Engineering in Medicine and Biology Society. Conference, volume 2013, pages 6567-70, January 2013. 


\section{Bibliography}

[ADP14] K. Aho, D. Derryberry, and T. Peterson. Model selection for ecologists: The worldviews of AIC and BIC. Ecology, 95(March):631-636, 2014.

[AGLE99] J. Arnhold, P. Grassberger, K. Lehnertz, and C. E. Elger. A robust method for detecting interdependences: application to intracranially recorded EEG. Physica D: Nonlinear Phenomena, 134(4):419-430, December 1999.

$\left[\mathrm{AGU}^{+} 81\right] \quad$ S. Akselrod, D. Gordon, F. Ubel, D. Shannon, A. Berger, and R. Cohen. Power spectrum analysis of heart rate fluctuation: a quantitative probe of beat-to-beat cardiovascular control. Science, 213(4504):220-222, July 1981.

$\left[\mathrm{AJI}^{+} 02\right]$ A. A. Armoundas, K. Ju, N. Iyengar, J. K. Kanters, P. J. Saul, R. J. Cohen, and K. H. Chon. A Stochastic Nonlinear Autoregressive Algorithm Reflects Nonlinear Dynamics of Heart-Rate Fluctuations. Annals of Biomedical Engineering, 30(2):192-201, February 2002.

[AK11] R. G. Andrzejak and T. Kreuz. Characterizing unidirectional couplings between point processes and flows. EPL (Europhysics Letters), 96(5):50012, December 2011.

[Aka69] H. Akaike. Fitting autoregressive models for prediction. Annals of the Institute of Statistical Mathematics, 21(1):243-247, December 1969.

[ALD06] R. G. Andrzejak, A. Ledberg, and G. Deco. Detecting event-related timedependent directional couplings. New Journal of Physics, 8(6):6-6, January 2006.

[AMS04] N. Ancona, D. Marinazzo, and S. Stramaglia. Radial basis function approach to nonlinear Granger causality of time series. Physical Review E: Statistical Physics, Plasmas, Fluids, and Related Interdisciplinary Topics, 70(5):1-7, November 2004.

[Bak70] D. W. Baker. Pulsed Ultrasonic Doppler Blood-Flow Sensing. IEEE Transactions on Sonics and Ultrasonics, 17(3):170-184, July 1970.

[BBSK02] V. Belozeroff, R. B. Berry, C. S. H. Sassoon, and M. C. K. Khoo. Effects of CPAP therapy on cardiovascular variability in obstructive sleep apnea: a closedloop analysis. American journal of physiology. Heart and circulatory physiology, 282(1):H110-21, January 2002.

$\left[\mathrm{BCB}^{+} 94\right]$ G. Baselli, S. Cerutti, F. Badilini, L. Biancardi, A. Porta, M. Pagani, F. Lombardi, O. Rimoldi, R. Furlan, and A. Malliani. Model for the assessment of heart period and arterial pressure variability interactions and of respiration influences. Medical ES biological engineering $\& 3$ computing, 32(2):143-52, March 1994.

[Bel57] R. E. Bellman. Dynamic Programming. Princeton University Press, 1957. 
[Bir31] G. D. Birkhoff. Proof of the Ergodic Theorem. Proceedings of the National Academy of Sciences of the United States of America, 17(3):656-660, 1931.

$\left[\mathrm{BKO}^{+} 08\right] \quad$ M. Barantke, T. Krauss, J. Ortak, W. Lieb, M. Reppel, C. Burgdorf, P. P. Pramstaller, H. Schunkert, and H. Bonnemeier. Effects of gender and aging on differential autonomic responses to orthostatic maneuvers. Journal of Cardiovascular Electrophysiology, 19(12):1296-303, December 2008.

$\left[\mathrm{BOL}^{+} 07\right] \quad$ M. Barantke, J. Ortak, W. Lieb, I. K. Wilke, H. Schunkert, and H. Bonnemeier. Effects of aging on reflex autonomic nervous response induced by orthostatic maneuvers. Pacing and Clinical Electrophysiology: PACE, 30 Suppl 1(January):S198202, January 2007.

[BP02] C. Bandt and B. Pompe. Permutation entropy: A natural complexity measure for time series. Physical Review Letters, 88(17):1-4, April 2002.

[BPRP03] B. P. Bezruchko, V. Ponomarenko, M. G. Rosenblum, and A. S. Pikovsky. Characterizing direction of coupling from experimental observations. Chaos: An Interdisciplinary Journal of Nonlinear Science, 13(1):179, 2003.

[BS01] L. A. Baccalá and K. Sameshima. Partial directed coherence: a new concept in neural structure determination. Biological Cybernetics, 84(6):463-74, June 2001.

$\left[\mathrm{BWB}^{+} 03\right]$ H. Bonnemeier, U. K. H. Wiegand, A. Brandes, N. Kluge, H. A. Katus, G. Richardt, and J. Potratz. Circadian profile of cardiac autonomic nervous modulation in healthy subjects: differing effects of aging and gender on heart rate variability. Journal of Cardiovascular Electrophysiology, 14(8):791-9, August 2003 .

$\left[\mathrm{BWH}^{+} 02\right] \quad$ M. Baumert, T. Walther, J. Hopfe, H. Stepan, R. Faber, and A. Voss. Joint symbolic dynamic analysis of beat-to-beat interactions of heart rate and systolic blood pressure in normal pregnancy. Medical \& Biological Engineering \& Computing, 40(2):241-5, March 2002.

[CA09] D. Chicharro and R. G. Andrzejak. Reliable detection of directional couplings using rank statistics. Physical Review E: Statistical Physics, Plasmas, Fluids, and Related Interdisciplinary Topics, 80(2):1-5, August 2009.

[CB96] S. Cavalcanti and E. Belardinelli. Modeling of cardiovascular variability using a differential delay equation. IEEE transactions on bio-medical engineering, 43(10):982-9, October 1996.

[CFB $\left.{ }^{+} 93\right] \quad$ P. J. Counihan, L. Fei, Y. Bashir, T. G. Farrell, G. A. Haywood, and W. J. McKenna. Assessment of heart rate variability in hypertrophic cardiomyopathy. Association with clinical and prognostic features. Circulation, 88(4):1682-1690, October 1993. 
$\left[\mathrm{CMT}^{+} 97\right]$ K. H. Chon, R. Mukkamala, K. Toska, T. J. Mullen, A. A. Armoundas, and R. J. Cohen. Linear and nonlinear system identification of autonomic heart-rate modulation. IEEE Engineering in Medicine and Biology Magazine, 16(5):96-105, 1997.

$\left[\mathrm{CRA}^{+} 14\right]$ S. Camargo, M. Riedl, C. Anteneodo, J. Kurths, T. Penzel, and N. Wessel. Sleep apnea-hypopnea quantification by cardiovascular data analysis. PloS one, 9(9):e107581, January 2014.

$\left[\mathrm{CRF}^{+}\right.$03] L. Cimponeriu, M. G. Rosenblum, T. Fieseler, J. Dammers, M. Schiek, M. Majtanik, P. Morosan, A. Bezerianos, and P. A. Tass. Inferring asymmetric relations between interacting neuronal oscillators. Progress of Theoretical Physics Supplement, 150(150):22-36, 2003.

[CRFD04] Y. Chen, G. Rangarajan, J. Feng, and M. Ding. Analyzing multiple nonlinear time series with extended Granger causality. Physics Letters A, 324(1):26-35, April 2004.

[CT93] R. Chen and R. S. Tsay. Nonlinear additive ARX models. Journal of the American Statistical Association, 1993.

[CT02] M. A. Cohen and J. A. Taylor. Short-term cardiovascular oscillations in man: measuring and modelling the physiologies. The Journal of Physiology, 542(3):669683, June 2002.

[CTK78] E. Callaway, P. Tueting, and S. H. Koslow, editors. Event-Related Brain Potentials in Man. Academic Press, Inc., New York, 1978.

[DB92] C. M. Dougherty and R. L. Burr. Comparison of heart rate variability in survivors and nonsurvivors of sudden cardiac arrest. The American journal of cardiology, 70(4):441-8, August 1992.

[DBR00] A. N. Dalgleish, G. S. Boulton, and E. Renshaw. The ice age cycle and the deglaciations: An application of nonlinear regression modelling. Quaternary Science Reviews, 19:687-697, 2000.

[DKS87] R. W. DeBoer, J. M. Karemaker, and J. Strackee. Hemodynamic fluctuations and baroreflex sensitivity in humans: a beat-to-beat model. The American journal of physiology, 253(3 Pt 2):H680-9, September 1987.

[DLK01] M. Dhamala, Y. C. Lai, and E. J. Kostelich. Analyses of transient chaotic time series. Physical review. E, Statistical, nonlinear, and soft matter physics, 64:056207, 2001.

[Ein03] W. Einthoven. Die galvanometrische Registrierung des menschlichen Elektrokardiogramms, zugleich eine Beurteilung der Anwendung des Capillarelektrometers in der Physiologie. Pflügers Archiv: European Journal of Physiology, 99:472-480, 1903. 
$\left[\right.$ ELS $\left.^{+} 00\right] \quad$ A. Effern, K. Lehnertz, T. Schreiber, T. Grunwald, P. David, and C. E. Elger. Nonlinear denoising of transient signals with application to event-related potentials. Physica D: Nonlinear Phenomena, 140:257-266, 2000.

$\left[F^{+} 12\right] \quad$ J. H. Feldhoff, R. V. Donner, J. F. Donges, N. Marwan, and J. Kurths. Geometric detection of coupling directions by means of inter-system recurrence networks. Physics Letters A, 376(46):3504-3513, October 2012.

[FNC08] L. Faes, G. Nollo, and K. H. Chon. Assessment of Granger causality by nonlinear model identification: application to short-term cardiovascular variability. Annals of Biomedical Engineering, 36(3):381-95, March 2008.

[FNP11] L. Faes, G. Nollo, and A. Porta. Information-based detection of nonlinear Granger causality in multivariate processes via a nonuniform embedding technique. Physical Review E: Statistical Physics, Plasmas, Fluids, and Related Interdisciplinary Topics, 83(5):1-15, May 2011.

[FNP12] L. Faes, G. Nollo, and A. Porta. Non-uniform multivariate embedding to assess the information transfer in cardiovascular and cardiorespiratory variability series. Computers in Biology and Medicine, 42(3):290-297, March 2012.

[FP07] S. Frenzel and B. Pompe. Partial mutual information for coupling analysis of multivariate time series. Physical Review Letters, 99(20):1-4, November 2007.

[FPN08] L. Faes, A. Porta, and G. Nollo. Mutual nonlinear prediction as a tool to evaluate coupling strength and directionality in bivariate time series: Comparison among different strategies based on $\mathrm{k}$ nearest neighbors. Physical Review E: Statistical Physics, Plasmas, Fluids, and Related Interdisciplinary Topics, 78(2):1-11, August 2008.

[FST97] R. H. Fagard, J. A. Staessen, and L. Thijs. Prediction of Cardiac Structure and Function by Repeated Clinic and Ambulatory Blood Pressure. Hypertension, 29(1):22-29, January 1997.

[Gal86] F. Galton. Regression towards mediocrity in hereditary stature. Journal of the Anthropological Institute of Great Britain and Ireland, 15:246-263, 1886.

[GBEC95] I. Gussak, P. Bjerregaard, T. M. Egan, and B. R. Chaitman. ECG phenomenon called the J wave. History, pathophysiology, and clinical significance. Journal of electrocardiology, 28(1):49-58, January 1995.

[Gew84] J. F. Geweke. Measures of conditional linear dependence and feedback between time series. Journal of the American Statistical Association, 79(388):907-915, 1984 .

[GFPK84] R. F. Gillum, S. P. Fortmann, R. J. Prineas, and T. E. Kottke. International diagnostic criteria for acute myocardial infarction and acute stroke. American Heart Journal, 108(1):150-8, July 1984. 
[Gra63] C. W. J. Granger. Economic processes involving feedback. Information and Control, 6(1):28-48, March 1963.

[Gra69] C. W. J. Granger. Investigating causal relations by econometric models and crossspectral methods. Econometrica, 37(3):424-438, 1969.

[Gro59] F. S. Grodins. Integrative cardiovascular physiology: a mathematical synthesis of cardiac and blood vessel hemodynamics. The Quarterly review of biology, 34(2):93-116, June 1959.

[GRS $\left.{ }^{+} 11\right]$ A. Gapelyuk, M. Riedl, A. Suhrbier, J. F. Kraemer, G. Bretthauer, H. Malberg, J. Kurths, T. Penzel, and N. Wessel. Cardiovascular regulation in different sleep stages in the obstructive sleep apnea syndrome. Biomedizinische Technik. Biomedical Engineering, 56(4):207-13, August 2011.

$\left[\mathrm{GSK}^{+} 08\right] \quad$ S. Guo, A. K. Seth, K. M. Kendrick, C. Zhou, and J. Feng. Partial Granger causality-eliminating exogenous inputs and latent variables. Journal of Neuroscience Methods, 172(1):79-93, July 2008.

[HA10] Y. Hirata and K. Aihara. Identifying hidden common causes from bivariate time series: A method using recurrence plots. Physical Review E: Statistical Physics, Plasmas, Fluids, and Related Interdisciplinary Topics, 81(1):1-7, January 2010.

[Hal33] S. Hales. Statical Essays: Containing Haemastaticks: Or, an Account of Some Hydraulick and Hydrostatical Experiments Made on the Blood and Blood Vessels of Animals. W. Innys and R. Manby, London, 1733.

[Har28] W. Harvey. Exercitatio Anatomica de Motu Cordis et Sanguinis in Animalibus. William Fitzer, Frankfurt, 1628.

$\left[\mathrm{HKvdL}^{+} 94\right]$ S. H. Hohnloser, T. Klingenheben, A. van de Loo, E. Hablawetz, H. Just, and P. J. Schwartz. Reflex versus tonic vagal activity as a prognostic parameter in patients with sustained ventricular tachycardia or ventricular fibrillation. Circulation, 89(3):1068-1073, March 1994.

[HSKM02] T. Heldt, E. B. Shim, R. D. Kamm, and R. G. Mark. Computational modeling of cardiovascular response to orthostatic stress. Journal of Applied Physiology (Bethesda, Md.: 1985), 92(3):1239-54, March 2002.

[HSPVB07] K. Hlaváčková-Schindler, M. Paluš, M. Vejmelka, and J. Bhattacharya. Causality detection based on information-theoretic approaches in time series analysis. Physics Reports, 441(1):1-46, March 2007.

$\left[\mathrm{HTK}^{+} 11\right] \quad$ P. A. Heidenreich, J. G. Trogdon, O. A. Khavjou, J. Butler, K. Dracup, M. D. Ezekowitz, E. A. Finkelstein, Y. Hong, S. C. Johnston, A. Khera, D. M. LloydJones, S. A. Nelson, G. Nichol, D. Orenstein, P. W. F. Wilson, and Y. J. Woo. Forecasting the future of cardiovascular disease in the United States: a policy statement from the American Heart Association. Circulation, 123(8):933-44, March 2011. 
[HW03] J. Huppelsberg and K. Walter. Kurzlehrbuch Physiologie. Thieme, Stuttgart, 2003.

[IAICQ07] C. Iber, S. Ancoli-Israel, A. Chesson, and S. F. Quan. The AASM Manual for the Scoring of Sleep and Associated Events: Rules, Terminology and Technical Specifications. American Academy of Sleep Medicine, 1st editio edition, 2007.

[IOLK08a] K. Ishiguro, N. Otsu, M. Lungarella, and Y. Kuniyoshi. Comparison of nonlinear Granger causality extensions for low-dimensional systems. Physical Review E: Statistical Physics, Plasmas, Fluids, and Related Interdisciplinary Topics, 77(3):19, March 2008.

[IOLK08b] K. Ishiguro, N. Otsu, M. Lungarella, and Y. Kuniyoshi. Detecting direction of causal interactions between dynamically coupled signals. Physical Review E: Statistical Physics, Plasmas, Fluids, and Related Interdisciplinary Topics, 77(2):2-7, February 2008.

[JIO ${ }^{+99}$ W. T. Jellema, B. P. M. Imholz, H. Oosting, K. H. Wesseling, and J. J. Van Lieshout. Estimation of beat-to-beat changes in stroke volume from arterial pressure: A comparison of two pressure wave analysis techniques during head- up tilt testing in young, healthy men. Clinical Autonomic Research, 9:185-192, 1999.

[JT94] I. Jánosi and T. Tél. Time-series analysis of transient chaos, 1994.

[KES $\left.{ }^{+} 04\right] \quad$ M. A. Kramer, E. Edwards, M. Soltani, M. S. Berger, R. T. Knight, and A. J. Szeri. Synchronization measures of bursting data: Application to the electrocorticogramof an auditory event-related experiment. Physical Review E: Statistical Physics, Plasmas, Fluids, and Related Interdisciplinary Topics, 70(1):1-10, July 2004 .

[KHI03] J. W. Kantelhardt, S. Havlin, and P. C. Ivanov. Modeling transient correlations in heartbeat dynamics during sleep. Europhysics Letters (EPL), 62(2):147-153, April 2003.

[Kli97] R. Klinge. Das Elektrokardiogramm: Leitfaden für Ausbildung und Praxis. Thieme, 1997.

$\left[\mathrm{KLY}^{+} 99\right]$ T. B. J. Kuo, T. Lin, C. C. Yang, C. L. Li, C. F. Chen, and P. Chou. Effect of aging on gender differences in neural control of heart rate. The American journal of physiology, 277(6 Pt 2):H2233-9, December 1999.

[KN06] J.-P. Kreißand G. Neuhaus. Einführung in die Zeitreihenanalyse. Springer Berlin Heidelberg, Berlin, Heidelberg, 2006.

[KOA $\left.{ }^{+} 05\right]$ M. Kikuya, T. Ohkubo, K. Asayama, H. Metoki, T. Obara, S. Saito, J. Hashimoto, K. Totsune, H. Hoshi, H. Satoh, and Y. Imai. Ambulatory blood pressure and 10year risk of cardiovascular and noncardiovascular mortality: the Ohasama study. Hypertension, 45(2):240-5, February 2005. 
[KS03] H. Kantz and T. Schreiber. Nonlinear time series analysis. Cambridge University Press, 2003.

$\left[\mathrm{KSA}^{+} 04\right] \quad$ K. Kiyono, Z. Struzik, N. Aoyagi, S. Sakata, J. Hayano, and Y. Yamamoto. Critical Scale Invariance in a Healthy Human Heart Rate. Physical Review Letters, 93(17):178103, October 2004.

$\left[\mathrm{KST}^{+} 05\right] \quad$ K. Kotani, Z. Struzik, K. Takamasu, H. Stanley, and Y. Yamamoto. Model for complex heart rate dynamics in health and diseases. Physical Review E, 72(4):041904, October 2005.

$\left[\mathrm{KTR}^{+} 08\right] \quad$ C. Komalapriya, M. Thiel, M. C. Romano, N. Marwan, U. Schwarz, and J. Kurths. Reconstruction of a system's dynamics from short trajectories. Physical Review E - Statistical, Nonlinear, and Soft Matter Physics, 78:1-11, 2008.

[Kuu04] T. Kuusela. Stochastic heart-rate model can reveal pathologic cardiac dynamics. Physical Review E, 69(3):031916, March 2004.

[LB01] R. S. T. Leung and T. D. Bradley. State of the Art Sleep Apnea and Cardiovascular Disease. American journal of respiratory and critical care medicine, 164:2147$2165,2001$.

[Leh11] K. Lehnertz. Assessing directed interactions from neurophysiological signals-an overview. Physiological Measurement, 32(11):1715-24, November 2011.

[Lev10] J. R. Levick. An Introduction to Cardiovascular Physiology. Hodder Arnold, 5th edition, 2010.

[Lew20] T. Lewis. The mechanism and graphic registration of the heart beat. Shaw \& Sons, London, 1920.

[LIKO07] M. Lungarella, K. Ishiguro, Y. Kuniyoshi, and N. Otsu. Methods for quantifying the causal structure of bivariate time series. International Journal of Bifurcation and Chaos, 17(3):903, 2007.

[LMAV99] M. Le Van Quyen, J. Martinerie, C. Adam, and F. J. Varela. Nonlinear analyses of interictal EEG map the brain interdependences in human focal epilepsy. Physica D: Nonlinear Phenomena, 127(3-4):250-266, March 1999.

[Lom00] F. Lombardi. Chaos Theory, Heart Rate Variability, and Arrhythmic Mortality. Circulation, 101(1):8-10, January 2000.

[LPK07] M. Lungarella, A. Pitti, and Y. Kuniyoshi. Information transfer at multiple scales. Physical Review E: Statistical Physics, Plasmas, Fluids, and Related Interdisciplinary Topics, 76(5):1-10, November 2007.

[Lud45] C. F. W. Ludwig. Einige Bemerkungen zu Valentins Lehren vom Athmen und vom Blutkreislauf. Zeitschrift für rationelle Medicin, 3(147), 1845. 
[LV76] B. Lown and R. L. Verrier. Neural activity and ventricular fibrillation. The New England journal of medicine, 294(21):1165-70, May 1976.

[LW08] S. Leski and D. Wójcik. Inferring coupling strength from event-related dynamics. Physical Review E: Statistical Physics, Plasmas, Fluids, and Related Interdisciplinary Topics, 78(4):1-9, October 2008.

[Mal98] M. Malik. Heart rate variability. Current Opinion in Cardiology, 13(1), 1998.

[May76] S. Mayer. Studien zur Physiologie des Herzens und der Blutgefässe. 6. Abhandlung: Über spontane Blutdruckschwankungen. Sitzungsberichte Akademie der Wissenschaften in Wien. Mathematisch-naturwissenschaftliche Classe, Anatomie, 74:281-307, 1876.

[MBMK12] N. Malik, B. Bookhagen, N. Marwan, and J. Kurths. Analysis of spatial and temporal extreme monsoonal rainfall over South Asia using complex networks. Climate Dynamics, 39:971-987, 2012.

[MCPR03] R. Mrowka, L. Cimponeriu, A. Patzak, and M. G. Rosenblum. Directionality of coupling of physiological subsystems: age-related changes of cardiorespiratory interaction during different sleep stages in babies. American Journal of Physiology: Regulatory, Integrative and Comparative Physiology, 285(6):R1395-401, December 2003.

$\left[\mathrm{MFG}^{+} 83\right]$ G. Mancia, A. Ferrari, L. Gregorini, G. Parati, G. Pomidossi, G. Bertinieri, G. Grassi, M. di Rienzo, A. Pedotti, and A. Zanchetti. Blood pressure and heart rate variabilities in normotensive and hypertensive human beings. Circulation Research, 53(1):96-104, July 1983.

[Mil51] R. H. Miller. Sphygmomanometer, 1951.

[MKWL11] M. Martini, T. A. Kranz, T. Wagner, and K. Lehnertz. Inferring directional interactions from transient signals with symbolic transfer entropy. Physical Review E: Statistical Physics, Plasmas, Fluids, and Related Interdisciplinary Topics, 83(1):1-6, January 2011.

[MLCS11] D. Marinazzo, W. Liao, H. Chen, and S. Stramaglia. Nonlinear connectivity by Granger causality. NeuroImage, 58(2):330-8, September 2011.

$\left[\mathrm{MMB}^{+}\right.$06] P. A. Modesti, M. Morabito, I. Bertolozzi, L. Massetti, G. Panci, C. Lumachi, A. Giglio, G. Bilo, G. Caldara, L. Lonati, S. Orlandini, G. Maracchi, G. Mancia, G. F. Gensini, and G. Parati. Weather-related changes in 24-hour blood pressure profile: effects of age and implications for hypertension management. Hypertension, 47(2):155-61, February 2006.

$\left[\mathrm{MOP}^{+} 01\right]$ G. Mancia, S. Omboni, G. Parati, D. L. Clement, W. E. Haley, S. N. Rahman, and R. P. Hoogma. Twenty-four hour ambulatory blood pressure in the Hypertension Optimal Treatment (HOT) study. Journal of hypertension, 19(10):1755-63, October 2001. 
[MOR $\left.{ }^{+} 95\right] \quad$ G. Mancia, S. Omboni, A. Ravogli, G. Parati, and A. Zanchetti. Ambulatory blood pressure monitoring in the evaluation of antihypertensive treatment: additional information from a large data base. Blood pressure, 4(3):148-56, May 1995.

$\left[\mathrm{MPB}^{+} 07\right]$ G. Mancia, G. Parati, G. Bilo, A. Maronati, S. Omboni, H. Baurecht, M. Hennig, and A. Zanchetti. Assessment of long-term antihypertensive treatment by clinic and ambulatory blood pressure: data from the European Lacidipine Study on Atherosclerosis. Journal of hypertension, 25(5):1087-94, May 2007.

$\left[\mathrm{MPH}^{+} 01\right]$ G. Mancia, G. Parati, M. Hennig, B. Flatau, S. Omboni, F. Glavina, B. Costa, R. Scherz, G. Bond, and A. Zanchetti. Relation between blood pressure variability and carotid artery damage in hypertension: baseline data from the European Lacidipine Study on Atherosclerosis (ELSA). Journal of hypertension, 19(11):1981-9, November 2001.

[MPR00] R. Mrowka, A. Patzak, and M. G. Rosenblum. Quantitative analysis of cardiorespiratory synchronization in infants. International Journal of Bifurcation and Chaos, 10(11):2479-2488, 2000.

[MPS08a] D. Marinazzo, M. Pellicoro, and S. Stramaglia. Kernel-Granger causality and the analysis of dynamical networks. Physical Review E: Statistical Physics, Plasmas, Fluids, and Related Interdisciplinary Topics, 77(5):056215, May 2008.

[MPS08b] D. Marinazzo, M. Pellicoro, and S. Stramaglia. Kernel Method for Nonlinear Granger Causality. Physical Review Letters, 100(14):1-4, April 2008.

$\left[\mathrm{MSM}^{+} 07\right] \quad$ B. Musizza, A. Stefanovska, P. V. E. McClintock, M. Paluš, J. Petrovčič, S. Ribarič, and F. F. Bajrović. Interactions between cardiac, respiratory and EEG-delta oscillations in rats during anaesthesia. The Journal of Physiology, 580(Pt 1):31526, April 2007.

[MW97] S. Matsukawa and T. Wada. Vector autoregressive modeling for analyzing feedback regulation between heart rate and blood pressure. The American journal of physiology, 273(1 Pt 2):H478-86, July 1997.

$\left[\mathrm{MWH}^{+} 02\right]$ H. Malberg, N. Wessel, A. Hasart, K.-J. Osterziel, and A. Voss. Advanced analysis of spontaneous baroreflex sensitivity, blood pressure and heart rate variability in patients with dilated cardiomyopathy. Clinical science (London, England: 1979), 102(4):465-73, April 2002.

$\left[\mathrm{MWM}^{+} 02\right]$ N. Marwan, N. Wessel, U. Meyerfeldt, A. Schirdewan, and J. Kurths. Recurrenceplot-based measures of complexity and their application to heart-rate-variability data. Physical Review E, 66(2):026702, August 2002.

$\left[\mathrm{MZW}^{+} 13\right]$ N. Marwan, Y. Zou, N. Wessel, M. Riedl, and J. Kurths. Estimating coupling directions in the cardiorespiratory system using recurrence properties. Philosophical transactions. Series A, Mathematical, physical, and engineering sciences, 371(1997):20110624, 2013. 
[NFAP09] G. Nollo, L. Faes, R. Antolini, and A. Porta. Assessing causality in normal and impaired short-term cardiovascular regulation via nonlinear prediction methods. Philosophical Transactions of the Royal Society A: Mathematical, Physical, and Engineering Sciences, 367(1892):1423-40, April 2009.

$\left[\mathrm{NFP}^{+} 05\right] \quad$ G. Nollo, L. Faes, A. Porta, R. Antolini, and F. Ravelli. Exploring directionality in spontaneous heart period and systolic pressure variability interactions in humans: implications in the evaluation of baroreflex gain. American Journal of Physiology: Heart and Circulatory Physiology, 288(4):H1777-85, April 2005.

[NR07] J. E. Naschitz and I. Rosner. Orthostatic hypotension: framework of the syndrome. Postgraduate medical journal, 83(983):568-574, 2007.

[NVF02] E. Nagel, A. C. Van Rossum, and E. Fleck. Kardiovaskuläre Magnetresonanztomographie: Methodenverständnis und praktische Anwendung. Steinkopff Darmstadt, 2002 .

[OPK $\left.{ }^{+} 00\right] \quad$ M. S. Olufsen, C. S. Peskin, W. Y. Kim, E. M. Pedersen, A. Nadim, and J. Larsen. Numerical Simulation and Experimental Validation of Blood Flow in Arteries with Structured-Tree Outflow Conditions. Annals of Biomedical Engineering, 28(11):1281-1299, November 2000.

[OS03] H. Oxenham and N. Sharpe. Cardiovascular aging and heart failure. European journal of heart failure, 5(4):427-34, August 2003.

[Ott97] J. T. Ottesen. Modelling of the baroreflex-feedback mechanism with time-delay. Journal of Mathematical Biology, 36(1):41-63, November 1997.

[Pal96] M. Paluš. Coarse-grained entropy rates for characterization of complex time series. Physica D: Nonlinear Phenomena, 93(1-2):64-77, May 1996.

[Pal07] M. Paluš. From nonlinearity to causality: statistical testing and inference of physical mechanisms underlying complex dynamics. Contemporary Physics, 48(6):307348, November 2007.

$\left[\mathrm{PBR}^{+} 00\right]$ A. Porta, G. Baselli, O. Rimoldi, A. Malliani, and M. Pagani. Assessing baroreflex gain from spontaneous variability in conscious dogs: role of causality and respiration. American Journal of Physiology: Heart and Circulatory Physiology, 279:H2558-H2567, 2000.

[Pea95] K. Pearson. Notes on regression and inheritance in the case of two parents. Proceedings of the Royal Society of London, 58:240-242, 1895.

[Pen73] J. Penaz. Photoelectric measurement of blood pressure, volume and flow in the finger. In Digest of the 10th international conference on medical and biological engineering, page 104, 1973. 
[PF13] A. Porta and L. Faes. Assessing causality in brain dynamics and cardiovascular control. Philosophical transactions. Series A, Mathematical, physical, and engineering sciences, 371(1997):20120517, August 2013.

$\left[\mathrm{PFM}^{+} 07\right]$ A. Porta, L. Faes, M. Masé, G. D’Addio, G. D. Pinna, R. Maestri, N. Montano, R. Furlan, S. Guzzetti, G. Nollo, and A. Malliani. An integrated approach based on uniform quantization for the evaluation of complexity of short-term heart period variability: Application to $24 \mathrm{~h}$ Holter recordings in healthy and heart failure humans. Chaos (Woodbury, N.Y.), 17(1):015117, March 2007.

$\left[\mathrm{PFR}^{+}\right.$02] A. Porta, R. Furlan, O. Rimoldi, M. Pagani, A. Malliani, and P. van de Borne. Quantifying the strength of the linear causal coupling in closed loop interacting cardiovascular variability signals. Biological Cybernetics, 86(3):241-251, March 2002 .

[PKHv01] M. Paluš, V. Komárek, Z. Hrnčǐř, and K. Štěrbová. Synchronization as adjustment of information rates: Detection from bivariate time series. Physical Review E: Statistical Physics, Plasmas, Fluids, and Related Interdisciplinary Topics, 63(4):16, March 2001.

[PKP $\left.{ }^{+} 01\right] \quad$ M. Paluš, V. Komárek, T. Procházka, Z. Hrnčǐr, and K. Štěrbová. Synchronization and information flow in EEGs of epileptic patients. IEEE Engineering in Medicine and Biology Magazine: The quarterly magazine of the Engineering in Medicine 8 Biology Society, 20(5):65-71, 2001.

[PLG $\left.{ }^{+} 86\right] \quad$ M. Pagani, F. Lombardi, S. Guzzetti, O. Rimoldi, R. Furlan, P. Pizzinelli, G. Sandrone, G. Malfatto, S. Dell'Orto, E. Piccaluga, M. Turiel, G. Baselli, S. Cerutti, and A. Malliani. Power spectral analysis of heart rate and arterial pressure variabilities as a marker of sympatho-vagal interaction in man and conscious dog. Circulation research, 59(2):178-93, August 1986.

[PR11] B. Pompe and J. Runge. Momentary information transfer as a coupling measure of time series. Physical Review E, 83(5):051122, May 2011.

$\left[\mathrm{PRG}^{+}{ }^{12}\right]$ T. Penzel, M. Riedl, A. Gapelyuk, A. Suhrbier, G. Bretthauer, H. Malberg, C. Schöbel, I. Fietze, J. Heitmann, J. Kurths, and N. Wessel. Effect of CPAP therapy on daytime cardiovascular regulations in patients with obstructive sleep apnea. Computers in biology and medicine, 42(3):328-34, March 2012.

[PRK01] A. S. Pikovsky, M. G. Rosenblum, and J. Kurths. Synchronization A Universal Concept in Nonlinear Sciences. Cambridge University Press, 2001.

[PS03] M. Paluš and A. Stefanovska. Direction of coupling from phases of interacting oscillators: An information-theoretic approach. Physical Review E: Statistical Physics, Plasmas, Fluids, and Related Interdisciplinary Topics, 67(5):1-4, May 2003. 
[PSV04] M. Paluš, A. Stefanovska, and M. Veber. Causality between the amplitude and frequency of cardiac oscillations. Cardiovascular Engineering, 4(2):127-132, June 2004 .

[PV07] M. Paluš and M. Vejmelka. Directionality of coupling from bivariate time series: How to avoid false causalities and missed connections. Physical Review E: Statistical Physics, Plasmas, Fluids, and Related Interdisciplinary Topics, 75(5):1-14, May 2007.

[QAG00] R. Quian Quiroga, J. Arnhold, and P. Grassberger. Learning driver-response relationships from synchronization patterns. Physical Review E: Statistical Physics, Plasmas, Fluids, and Related Interdisciplinary Topics, 61(5 Pt A):5142-8, May 2000 .

[QCKH11] C. J. Quinn, T. P. Coleman, N. Kiyavash, and N. G. Hatsopoulos. Estimating the directed information to infer causal relationships in ensemble neural spike train recordings. Journal of computational neuroscience, 30(1):17-44, February 2011.

[QKKG02] R. Quian Quiroga, A. Kraskov, T. Kreuz, and P. Grassberger. Performance of different synchronization measures in real data: A case study on electroencephalographic signals. Physical Review E: Statistical Physics, Plasmas, Fluids, and Related Interdisciplinary Topics, 65(4):1-14, March 2002.

[Ras86] F. Raschke. The hierarchical order of cardio-vascular-respiratory coupling. In P. Grossman, K. H. L. Janssen, and D. Vaitl, editors, Cardiorespiratory and Cardiosomatic Psychophysiology. Plenum, New York, 1986.

[Ras87] F. Raschke. Coordination in the circulatory and respiratory systems. In L. Resing, U. an der Heiden, and M. C. Mackey, editors, Temporal Disorder in Human Oscillatory Systems, pages 152-158. Springer, Berlin, 1987.

$\left[\mathrm{RCB}^{+} 02\right]$ M. G. Rosenblum, L. Cimponeriu, A. Bezerianos, A. Patzak, and R. Mrowka. Identification of coupling direction: Application to cardiorespiratory interaction. Physical Review E: Statistical Physics, Plasmas, Fluids, and Related Interdisciplinary Topics, 65(4):1-11, March 2002.

$\left[\mathrm{RDR}^{+} 13\right] \quad$ A. Radebach, R. V. Donner, J. Runge, J. F. Donges, and J. Kurths. Disentangling different types of El Niño episodes by evolving climate network analysis. Physical Review E - Statistical, Nonlinear, and Soft Matter Physics, 88:1-19, 2013.

[Rem05] J. E. Remmers. A century of control of breathing. American Journal of Respiratory and Critical Care Medicine, 172(1):6-11, July 2005.

[RGAC14] R. Romero-Garcia, M. Atienza, and J. L. Cantero. Predictors of coupling between structural and functional cortical networks in normal aging. Human brain mapping, 35(6):2724-40, June 2014. 
[RGM $\left.{ }^{+} 13\right]$ G. M. Ramírez Ávila, A. Gapelyuk, N. Marwan, T. Walther, H. Stepan, J. Kurths, and N. Wessel. Classification of cardiovascular time series based on different coupling structures using recurrence networks analysis., 2013.

[RH82] F. Raschke and G. Hildebrandt. Coupling of the cardiorespiratory control system by modulation and triggering. In T. Kenner, R. Busse, and H. Hinghofer-Szalkay, editors, Cardiovascular System Dynamics - Models and Measurements, pages 533542. Plenum, New York, 1982.

[RHMK12] J. Runge, J. Heitzig, N. Marwan, and J. Kurths. Quantifying causal coupling strength: A lag-specific measure for multivariate time series related to transfer entropy. Physical Review E, 86(6):061121, December 2012.

[RHPK12] J. Runge, J. Heitzig, V. Petoukhov, and J. Kurths. Escaping the Curse of Dimensionality in Estimating Multivariate Transfer Entropy. Physical Review Letters, 108(25):258701, June 2012.

[Rie09] M. Riedl. Model-based Analysis of Cardiovascular Interactions. PhD thesis, Universität Potsdam, 2009.

[RK95] M. G. Rosenblum and J. Kurths. A model of neural control of the heart rate. Physica A: Statistical Mechanics and its Applications, 215(4):439-450, May 1995.

$\left[\mathrm{RKP}^{+} 98\right] \quad$ M. G. Rosenblum, J. Kurths, A. S. Pikovsky, C. Schäfer, P. A. Tass, and H.-H. Abel. Synchronization in noisy systems and cardiorespiratory interaction. IEEE Engineering in Medicine and Biology Magazine: The quarterly magazine of the Engineering in Medicine \& Biology Society, 17(6):46-53, 1998.

[RP01] M. G. Rosenblum and A. S. Pikovsky. Detecting direction of coupling in interacting oscillators. Physical Review E: Statistical Physics, Plasmas, Fluids, and Related Interdisciplinary Topics, 64(4):2-5, September 2001.

[RPK $\left.{ }^{+} 01\right] \quad$ M. G. Rosenblum, A. S. Pikovsky, J. Kurths, C. Schäfer, and P. A. Tass. Phase synchronization: from theory to data analysis. Handbook of Biological Physics, 4(9):279-321, 2001.

$\left[\mathrm{RSM}^{+} 08\right] \quad$ M. Riedl, A. Suhrbier, H. Malberg, T. Penzel, G. Bretthauer, J. Kurths, and N. Wessel. Modeling the cardiovascular system using a nonlinear additive autoregressive model with exogenous input. Physical Review E: Statistical Physics, Plasmas, Fluids, and Related Interdisciplinary Topics, 78(1):1-9, July 2008.

$\left[\mathrm{RSS}^{+}\right.$10] M. Riedl, A. Suhrbier, H. Stepan, J. Kurths, and N. Wessel. Short-term couplings of the cardiovascular system in pregnant women suffering from pre-eclampsia. Philosophical Transactions of the Royal Society A: Mathematical, Physical, and Engineering Sciences, 368(1918):2237-50, May 2010.

[RTKG07] M. C. Romano, M. Thiel, J. Kurths, and C. Grebogi. Estimation of the direction of the coupling by conditional probabilities of recurrence. Physical Review E: 
Statistical Physics, Plasmas, Fluids, and Related Interdisciplinary Topics, 76(3):19, September 2007.

[SA05] D. A. Smirnov and R. G. Andrzejak. Detection of weak directional coupling: Phase-dynamics approach versus state-space approach. Physical Review E: Statistical Physics, Plasmas, Fluids, and Related Interdisciplinary Topics, 71(3):1-13, March 2005.

[SAE ${ }^{+13}$ S. Schulz, F.-C. Adochiei, I.-R. Edu, R. Schroeder, H. Costin, K.-J. Bär, and A. Voss. Cardiovascular and cardiorespiratory coupling analyses: a review. Philosophical transactions. Series A, Mathematical, physical, and engineering sciences, 371(1997):20120191, August 2013.

$\left[\mathrm{SAL}^{+}{ }^{89}\right] \quad$ K. E. Sands, M. L. Appel, L. S. Lilly, F. J. Schoen, G. H. Mudge, and R. J. Cohen. Power spectrum analysis of heart rate variability in human cardiac transplant recipients. Circulation, 79(1):76-82, January 1989.

[Say73] B. M. Sayers. Analysis of heart rate variability. Ergonomics, 16(1):17-32, January 1973.

[SB03] D. A. Smirnov and B. P. Bezruchko. Estimation of interaction strength and direction from short and noisy time series. Physical Review E: Statistical Physics, Plasmas, Fluids, and Related Interdisciplinary Topics, 68(4):1-10, October 2003.

[SB14] J. Sun and E. M. Bollt. Causation entropy identifies indirect influences, dominance of neighbors and anticipatory couplings. Physica D: Nonlinear Phenomena, 267:49-57, January 2014.

[SCB ${ }^{+98] ~ R . ~ S e g a, ~ G . ~ C e s a n a, ~ M . ~ B o m b e l l i, ~ G . ~ G r a s s i, ~ M . ~ L . ~ S t e l l a, ~ A . ~ Z a n c h e t t i, ~ a n d ~}$ G. Mancia. Seasonal variations in home and ambulatory blood pressure in the PAMELA population. Pressione Arteriose Monitorate E Loro Associazioni. Journal of hypertension, 16(11):1585-92, November 1998.

[SCB14] J. Sun, C. Cafaro, and E. Bollt. Identifying the Coupling Structure in Complex Systems through the Optimal Causation Entropy Principle. Entropy, 16(6):34163433, June 2014.

[Sch78] G. Schwarz. Estimating the dimension of a model. The Annals of Statistics, 6(2):461-464, 1978.

[Sch00a] A. Schmitz. Measuring statistical dependence and coupling of subsystems. Physical Review E: Statistical Physics, Plasmas, Fluids, and Related Interdisciplinary Topics, 62(5):7508-7511, November 2000.

[Sch00b] T. Schreiber. Measuring information transfer. Physical Review Letters, 85(2):4614, July 2000. 
[SDHK05] P. K. Stein, P. P. Domitrovich, H. V. Huikuri, and R. E. Kleiger. Traditional and nonlinear heart rate variability are each independently associated with mortality after myocardial infarction. Journal of cardiovascular electrophysiology, 16(1):1320, January 2005.

[Sha48] C. E. Shannon. A mathematical theory of communication. The Bell System Technical Journal, 27:379-423, 1948.

[SL08] M. Staniek and K. Lehnertz. Symbolic Transfer Entropy. Physical Review Letters, 100(15):1-4, April 2008.

[SL09a] M. Staniek and K. Lehnertz. Symbolic transfer entropy: inferring directionality in biosignals. Biomedizinische Technik. Biomedical Engineering, 54(6):323-8, December 2009.

[SL09b] S. Stausberg and K. Lehnertz. Nonlinear denoising of functional magnetic resonance imaging time series with wavelets. Physical Review E - Statistical, Nonlinear, and Soft Matter Physics, 79:1-8, 2009.

[SLM01] A. Stefanovska, D. G. Luchinsky, and P. V. E. McClintock. Modelling couplings among the oscillators of the cardiovascular system. Physiological Measurement, 22(3):551-564, August 2001.

[SLSH01] A. Stefanovska, M. B. Lotrič, S. Strle, and H. Haken. The cardiovascular system as coupled oscillators? Physiological Measurement, 22(3):535-50, August 2001.

[SM09] D. A. Smirnov and I. I. Mokhov. From Granger causality to long-term causality: Application to climatic data. Physical Review E: Statistical Physics, Plasmas, Fluids, and Related Interdisciplinary Topics, 80(1):1-11, July 2009.

$\left[\mathrm{SNG}^{+} 04\right] \quad$ S. A. Sheth, M. Nemoto, M. Guiou, M. Walker, N. Pouratian, and A. W. Toga. Linear and nonlinear relationships between neuronal activity, oxygen metabolism, and hemodynamic responses. Neuron, 42(2):347-55, April 2004.

[SRAK99] C. Schäfer, M. G. Rosenblum, H.-H. Abel, and J. Kurths. Synchronization in the human cardiorespiratory system. Physical Review E: Statistical Physics, Plasmas, Fluids, and Related Interdisciplinary Topics, 60(1):857-70, July 1999.

[SRKA98] C. Schäfer, M. G. Rosenblum, J. Kurths, and H.-H. Abel. Heartbeat synchronized with ventilation. Nature, 392(6673):239-40, March 1998.

$\left[\mathrm{SRM}^{+} 10\right]$ A. Suhrbier, M. Riedl, H. Malberg, T. Penzel, G. Bretthauer, J. Kurths, and N. Wessel. Cardiovascular regulation during sleep quantified by symbolic coupling traces. Chaos (Woodbury, N.Y.), 20(4):045124, December 2010.

$\left[\mathrm{SSC}^{+} 96\right] \quad$ S. J. Schiff, P. So, T. Chang, R. E. Burke, and T. Sauer. Detecting dynamical interdependence and generalized synchrony through mutual prediction in a neural ensemble. Physical Review E: Statistical Physics, Plasmas, Fluids, and Related Interdisciplinary Topics, 54(6):6708-6724, December 1996. 
[Sta18] E. H. Starling. The Linacre Lecture on the Law of the Heart Given at Cambridge, 1915, March 1918.

$\left[\mathrm{STB}^{+} 13\right] \quad$ S. Schulz, N. Tupaika, S. Berger, J. Haueisen, K.-J. Bär, and A. Voss. Cardiovascular coupling analysis with high-resolution joint symbolic dynamics in patients suffering from acute schizophrenia. Physiological measurement, 34(8):883-901, August 2013.

[STB14] J. Sun, D. Taylor, and E. M. Bollt. Causal Network Inference by Optimal Causation Entropy. SIAM Journal on Applied Dynamical Systems, 13699(61386):27, January 2014.

$\left[\mathrm{STF}^{+}\right.$99] J. A. Staessen, L. Thijs, R. Fagard, E. T. O'Brien, D. L. Clement, P. W. de Leeuw, G. Mancia, C. Nachev, P. Palatini, G. Parati, J. Tuomilehto, and J. Webster. Predicting cardiovascular risk using conventional vs ambulatory blood pressure in older patients with systolic hypertension. Systolic Hypertension in Europe Trial Investigators. JAMA, 282(6):539-46, August 1999.

[Sur95] B. Surawicz. Electrolytes, hormones, temperature, and miscellaneous factors: electrophysiologic basis of ECG and cardiac arrhythmias. Williams \& Wilkins, Baltimore, 1995.

$\left[\mathrm{SWD}^{+} 06\right] \quad$ B. O. Schelter, M. Winterhalder, R. Dahlhaus, J. Kurths, and J. Timmer. Partial phase synchronization for multivariate synchronizing systems. Physical Review Letters, 96(20), May 2006.

$\left[\mathrm{SWE}^{+} 06\right]$ B. O. Schelter, M. Winterhalder, M. Eichler, M. Peifer, B. Hellwig, B. Guschlbauer, C. H. Lücking, R. Dahlhaus, and J. Timmer. Testing for directed influences among neural signals using partial directed coherence. Journal of Neuroscience Methods, 152(1-2):210-9, April 2006.

[Tas96] Taskforce. Heart rate variability. Standards of measurement, physiological interpretation, and clinical use. Task Force of the European Society of Cardiology and the North American Society of Pacing and Electrophysiology. European Heart Journal, 17(3):354-81, March 1996.

[TB03] J. R. Terry and M. Breakspear. An improved algorithm for the detection of dynamical interdependence in bivariate time-series. Biological Cybernetics, 88(2):129-36, February 2003.

$\left[\mathrm{UBP}^{+} 01\right]$ E. Ullman, W. J. Brady, A. D. Perron, T. Chan, and A. Mattu. Electrocardiographic manifestations of pulmonary embolism. The American journal of emergency medicine, 19(6):514-9, October 2001.

[Vej08] M. Vejmelka. Quantifying interactions between complex oscillatory systems: A topic in time series analysis. PhD thesis, Czech Technical University in Prague, 2008 . 
[Ver38] P.-F. Verhulst. Notice sur la loi que la population poursuit dans son accroissement. Correspondance mathématique et physique, 10:113-121, 1838.

[Ver05] P. F. Verdes. Assessing causality from multivariate time series. Physical Review E: Statistical Physics, Plasmas, Fluids, and Related Interdisciplinary Topics, 72(2):19, August 2005.

[VK97] H. Voss and J. Kurths. Reconstruction of non-linear time delay models from data by the use of optimal transformations. Physics Letters A, 234:336-344, 1997.

[VK99] H. Voss and J. Kurths. Reconstruction of nonlinear time-delayed feedback models from optical data. Chaos, Solitons \& Fractals, 10(4-5):805-809, 1999.

[VKAK99] H. Voss, P. Kolodner, M. Abel, and J. Kurths. Amplitude Equations from Spatiotemporal Binary-Fluid Convection Data. Physical Review Letters, 83:34223425, 1999.

[VKK $\left.{ }^{+} 95\right]$ A. Voss, J. Kurths, H. J. Kleiner, A. Witt, and N. Wessel. Improved analysis of heart rate variability by methods of nonlinear dynamics. Journal of electrocardiology, 28 Suppl:81-8, January 1995.

[VKK ${ }^{+}$96] A. Voss, J. Kurths, H. J. Kleiner, A. Witt, N. Wessel, P. Saparin, K. J. Osterziel, R. Schurath, and R. Dietz. The application of methods of non-linear dynamics for the improved and predictive recognition of patients threatened by sudden cardiac death. Cardiovascular research, 31(3):419-33, March 1996.

[VP08] M. Vejmelka and M. Paluš. Inferring the directionality of coupling with conditional mutual information. Physical Review E: Statistical Physics, Plasmas, Fluids, and Related Interdisciplinary Topics, 77(2):1-12, February 2008.

[VSK04] C. Van Mieghem, M. Sabbe, and D. Knockaert. The clinical value of the ECG in noncardiac conditions. Chest, 125(4):1561-76, April 2004.

[VSKM99] H. U. Voss, A. Schwache, J. Kurths, and F. Mitschke. Equations of motion from chaotic data: A driven optical fiber ring resonator. Physics Letters A, 256(May):47-54, 1999.

[WFL10] T. Wagner, J. Fell, and K. Lehnertz. The detection of transient directional couplings based on phase synchronization. New Journal of Physics, 12(5):053031, May 2010.

[WHO10] WHO. Global status report on noncommunicable diseases 2010. Technical report, World Health Organization, 2010.

[WJSS93] K. H. Wesseling, J. R. Jansen, J. J. Settels, and J. J. Schreuder. Computation of aortic flow from pressure in humans using a nonlinear, three-element model. Journal of applied physiology (Bethesda, Md. : 1985), 74:2566-2573, 1993. 
[WKDB07] N. Wessel, J. Kurths, W. Ditto, and R. Bauernschmitt. Introduction: Cardiovascular physics. Chaos (Woodbury, N.Y.), 17(1):015101, March 2007.

$\left[\mathrm{WMB}^{+} 06\right]$ N. Wessel, H. Malberg, R. Bauernschmitt, A. Schirdewan, and J. Kurths. Nonlinear additive autoregressive model-based analysis of short-term heart rate variability. Medical $\mathscr{E}$ biological engineering $\mathscr{E}$ computing, 44(4):321-30, April 2006.

[WMBK07] N. Wessel, H. Malberg, R. Bauernschmitt, and J. Kurths. Nonlinear methods of cardiovascular physics and their clinical applicability. International Journal of Bifurcation and Chaos, 17(10):3325-3371, 2007.

[WSH ${ }^{+}$06] M. Winterhalder, B. O. Schelter, W. Hesse, K. Schwab, L. Leistritz, J. Timmer, and H. Witte. Detection of directed information flow in biosignals. Biomedizinische Technik. Biomedical Engineering, 51(5-6):281-7, December 2006.

[WSR ${ }^{+}$09] N. Wessel, A. Suhrbier, M. Riedl, N. Marwan, H. Malberg, G. Bretthauer, T. Penzel, and J. Kurths. Detection of time-delayed interactions in biosignals using symbolic coupling traces. Europhysics Letters, 87(10004):10004, July 2009.

[WST07] M. Winterhalder, B. O. Schelter, and J. Timmer. Detecting coupling directions in multivariate oscillatory systems. International Journal of Bifurcation and Chaos, 17(10):3735, 2007.

[Zan09] M. Zandi. Financial Shock. Pearson Education, Inc., 2009.

[ZF09] C. Zou and J. Feng. Granger causality vs. dynamic Bayesian network inference: a comparative study. BMC Bioinformatics, 10:122, January 2009.

$\left[\mathrm{ZGB}^{+} 07\right] \quad$ J. J. Zebrowski, K. Grudzinski, T. Buchner, P. Kuklik, J. Gac, G. Gielerak, P. Sanders, and R. Baranowski. Nonlinear oscillator model reproducing various phenomena in the dynamics of the conduction system of the heart. Chaos (Woodbury, N.Y.), 17(1):015121, March 2007.

$\left[\mathrm{ZRT}^{+} 11\right] \quad$ Y. Zou, M. C. Romano, M. Thiel, N. Marwan, and J. Kurths. Inferring Indirect Coupling By Means of Recurrences. International Journal of Bifurcation and Chaos, 21(04):1099, 2011. 


\section{Selbstständigkeitserklärung}

Hiermit erkläre ich, die Dissertation selbstständig und nur unter Verwendung der angegebenen Hilfen und Hilfsmittel angefertigt zu haben. 